\title{
Pacific
}

Journal of

Mathematics

\section{METRICS FOR SINGULAR ANALYTIC SPACES}

Caroline Galt Grant and Pierre Milman 


\section{METRICS FOR SINGULAR ANALYTIC SPACES}

\section{Caroline Grant and Pierre Milman}

Recent work of Saper, Zucker, and others indicates that Kähler metrics with appropriate growth rates on the nonsingular set of a compact Kähler variety are useful in describing the geometry of such a variety. It has been conjectured that for every complex algebraic variety $X$ there exists a Kähler metric on the nonsingular set of $X$ whose $L_{2}$-cohomology is isomorphic to the intersection cohomology of $X$. Saper proved this conjecture for varieties with isolated singularities, using a complete Kähler metric. Similar results have been obtained by others using incomplete metrics. We give natural and explicit constructions of three types of Kähler metrics on the nonsingular set $X-X_{\text {sing }}$ of a subvariety $X$ of a compact Kähler manifold. No restrictions on the type of singularities of $X$ are assumed. Similar constructions can be done for nonembedded compact Kähler varieties. The first metric is Hodge if $X$ is algebraic but is not complete on $X-X_{\text {sing }}$ if $X$ is singular. The completion of $X-X_{\operatorname{sing}}$ under this metric is a desingularization of $X$. The second metric is complete and generalizes Saper's metric for varieties with isolated singularities. Moreover each incomplete metric of the first type is naturally associated with a complete metric of the second type. The third metric is a sum of the first two and has Poincaré-type growth near the singular locus of $X$.

$\S 1$. Introduction. If $X$ is a smooth algebraic variety over $\mathbf{C}$, or more generally, a compact Kähler manifold, then the cohomology of $X$ with complex coefficients $H^{*}(X)$ is isomorphic to the de Rham cohomology $H_{\mathrm{DR}}^{*}(X)$ and satisfies a collection of cohomology theorems including Poincaré duality, the Lefschetz hyperplane theorem, and the hard Lefschetz theorem. These cohomology theorems do not always hold for $H^{*}(X)$ when $X$ is singular. In this case the appropriate cohomology is the intersection cohomology of Goresky-MacPherson. It has been conjectured that the appropriate replacement for the de Rham cohomology is $L_{2}$-cohomology, or more 
precisely, that the intersection cohomology of a singular algebraic variety $X$ is always isomorphic to the $L_{2}$-cohomology of $X$ with respect to a suitable Kähler metric on the nonsingular set $X-X_{\text {sing }}$ of $X$. Saper proved this conjecture for varieties with isolated singularities, using a complete Kähler metric [S2]. Recently Ohsawa [O] announced this conjecture for complex projective varieties using the induced incomplete metric. Complete Kähler metrics are of particular interest because they yield Hodge decompositions.

We construct three types of Kähler metrics on the nonsingular set $X-X_{\text {sing }}$ of a subvariety $X$ of a compact Kähler manifold. Our constructions are explicit and use in a natural way the geometry of a sequence of blow-ups used to resolve the singularities of $X$. No restrictions on the type of singularities are assumed. These metrics were introduced in $[\mathrm{GrM}]$. The first metric is induced by a metric which we construct on a desingularization $\tilde{X}$ of $X$ and has the property that it is Hodge if $X$ is algebraic. If $X$ is singular, this metric is not complete on $X-X_{\text {sing }}$ and the completion of $X-X_{\text {sing }}$ with respect to this metric is the desingularization $\tilde{X}$.

The second metric is complete on $X-X_{\text {sing }}$ and generalizes Saper's metric for varieties with isolated singularities. We show that each incomplete metric of the first type is naturally associated with a complete metric of this type. We also prove one of the conditions needed to apply Goresky-MacPherson's theorem [GM] characterizing intersection cohomology: we show that for this metric, the associated complex of $L_{2}$-bounded sheaves on $X$ is fine. This modified Saper metric seems to be a good candidate for extending Saper's theorem to more general varieties.

The third metric is the sum of our incomplete metric and our modified Saper metric. It is complete and has Poincaré-type growth near $X_{\text {sing. }}$. The $L_{2}$-cohomology of a true Poincaré metric on the complement of a divisor with normal crossings on $\tilde{X}$ is isomorphic to the intersection cohomology of $\tilde{X}$, not $X$. We expect the same to be true for our modified Poincaré metric. This metric is bounded below by the induced incomplete metric and the modified Saper metric. Consequently, the associated sheaves of $L_{2}$-bounded forms on both $X$ and $\tilde{X}$ are fine.

Although we have restricted ourselves in this paper to subvarieties of compact Kähler manifolds, similar constructions can be done in 
more general settings.

In an appendix, we include, for lack of a suitable reference, some tubular neighbourhood constructions which are based on a construction of Clemens [CL].

We will give further guidance to the paper in $\S 2$, after a few preliminaries.

We would like to acknowledge helpful conversations with John Bland, Michael Hoffman, and Boris Youssin.

\section{TABle of Contents}

\section{$\S 1$. Introduction}

$\S 2$. Main results and guidance through the paper

$\S 3$. Background on geometry of blow-ups

$\S 4$. An incomplete metric on $M-X_{\text {sing }}$ which determines an embedded resolution of $X$ by blow-ups

$\S 5$. Splitting of a Poincaré-type $(1,1)$-form into a sum of its two essential parts $\S 6$. Main ingredient of completeness of our metrics $\S 7$. Modified Saper and Poincaré metrics for a single blowup

$\S 8$. Modified Saper and Poincaré metrics for successive blow-ups and proof of Theorem I

$\S 9$. Homogeneous Saper Metrics and proof of Theorem II

\section{$\S 10$. Fine sheaves}

Appendix: Tubular neighbourhood construction

(1.1) Resolution of Singularities. Let $X$ be a reduced singular compact analytic subspace of a compact Kähler manifold $M$. Let $X_{\text {sing }}$ be the singular set of $X$. The singularities of $X$ may be resolved by a finite sequence of blow-ups $\pi_{j}: M_{j} \rightarrow M_{j-1}$ along smooth centres $C_{j}$ in $M_{j-1}$, where $M_{0}=M$. We will describe a systematic way of constructing a complete Kähler metric on $X-X_{\text {sing }}$, using the geometry of these blow-ups.

Let $X_{j}$ be the strict transform of $X$ in $M_{j}$, i.e. $X_{j}$ is the closure of $\pi_{j}^{-1}\left(X_{j-1}-C_{j}\right)$ in $M_{j}$. Let $D_{j}$ be the exceptional divisor of the 
composite $\pi_{1} \circ \pi_{2} \circ \ldots \circ \pi_{j}$ of the first $j$ blow-ups, i.e. $D_{1}=\pi_{1}^{-1}\left(C_{1}\right)$ and $D_{j}=\pi_{j}^{*} D_{j-1}+\pi_{j}^{-1}\left(C_{j}\right)$ for $j \geq 2$. Let $\tilde{M}$ be the final blow-up of $M, \tilde{X}$ the final strict transform of $X, D$ the final exceptional divisor, and $\pi: \tilde{M} \rightarrow M=M_{0}$ the composite of all the blow-ups. By the notation $\tilde{M}-D$ we will always mean $\tilde{M}-\operatorname{supp} D$.

Definition. We say that an analytic subspace $Z$ of a complex manifold $M$ has only normal crossings if, at each point $p$ in $Z$, there is a local coordinate system $\left(z_{1}, \ldots, z_{n}\right)$ on $M$ in which $Z$ is given by the vanishing of a collection of monomials $z_{1}^{\alpha_{1}} z_{2}^{\alpha_{2}} \ldots z_{n}^{\alpha_{n}}$.

Hironaka $[\mathbf{H}]$ showed that the centres $C_{j}$ may be chosen so that

(1) $C_{j}$ is contained in the singular locus $\left(X_{j-1}\right)_{\text {sing }}$ of $X_{j-1}$ if $X_{j-1}$ is singular, or in $X_{j-1} \cap D_{j-1}$ otherwise,

(2) $C_{j}$ and $D_{j-1}$ simultaneously have only normal crossings,

(3) $\tilde{X}$ is smooth, and

(4) $\tilde{X}$ and $D$ have normal crossings.

It follows from properties (1) - (4) that

(5) $D=\pi^{-1}\left(X_{\text {sing }}\right)$,

(6) the restriction of $\pi$ to $\tilde{M}-D$ is a biholomorphism onto $M$ $X_{\text {sing }}$ (and consequently the restriction to $\tilde{X}-(\tilde{X} \cap D)$ is a biholomorphism onto $\left.X-X_{\text {sing }}\right)$, and

(7) $\pi_{j, 0}^{-1}\left(X_{\text {sing }}\right) \supset\left(X_{j}\right)_{\text {sing }}$ where $\pi_{j, 0}$ is the composite map $\pi_{j, 0}=$ $\pi_{1} \circ \pi_{2} \circ \ldots \circ \pi_{j}: M_{j} \rightarrow M_{0}$.

Note: A simple proof of canonical resolution of singularities can be found in [BM2].

We will construct a complete Kähler metric on each noncompact manifold $M_{j}-D_{j}$. The restriction of the final metric on $\tilde{M}-D$ to $\tilde{X}-(\tilde{X} \cap D)$ induces a complete Kähler metric on $X-X_{\text {sing }}$.

(1.2) Metrics and their fundamental $(1,1)$-forms. Let $h$ be a hermitian metric on a complex manifold $M$. In local coordinates $\left(z_{1}, z_{2}, \ldots, z_{n}\right)$ on an open set $U$ in $M$, the metric $h$ can be written

$$
\left.h\right|_{U}=\sum_{i, j=1}^{n} h_{i j} d z_{i} \otimes d \bar{z}_{j}
$$

where $\left(h_{i j}\right)$ is a positive definite hermitian matrix whose entries are $C^{\infty}$ functions on $U$. Specifying a hermitian metric $h$ on $M$ is equivalent to specifying a positive hermitian $C^{\infty}(1,1)$-form $\omega$ on 
$M$, given locally by

$$
\left.\omega\right|_{U}=\frac{\sqrt{-1}}{2} \sum_{i, j=1}^{n} h_{i j} d z_{i} \wedge d \bar{z}_{j} .
$$

We call $\omega$ the fundamental form of $h$. The metric $h$ is called Kähler and $\omega$ is called a Kähler form if $\omega$ is $d$-closed, i.e. if $d \omega=0$. A Kähler metric is called Hodge and its Kähler form $\omega$ is called a Hodge form if $\omega$ is integral, i.e. if the de Rham cohomology class of $\omega$ lies in the image of $H^{*}(M, \mathbf{Z})$ under the natural map $H^{*}(M, \mathbf{Z}) \rightarrow$ $H_{D R}^{*}(M, \mathbf{C})$. The manifold $M$ is called Kähler (respectively Hodge) if it admits a Kähler (respectively Hodge) metric. The following will be useful in constructing Kähler forms.

LEMMA 1.2.1. If $g$ is a positive real $C^{\infty}$ function on an open set $U$ in $\mathbf{C}^{n}$, then the $(1,1)-$ form $\omega=\partial \bar{\partial} \log g$ is hermitian and $d$-closed on $U$.

Proof. It is easily checked that $\frac{\partial^{2} g}{\partial z_{i} \partial \bar{z}_{j}}=\overline{\frac{\partial^{2} g}{\partial z_{j} \partial \bar{z}_{i}}}$ for any two local coordinates $z_{i}$ and $z_{j}$ in $\mathbf{C}^{n}$ and that $d \partial \bar{\partial} \psi=0$ for any $C^{\infty}$ function $\psi$.

(1.3) Quasi-isometry. We say that two metrics $h_{1}$ and $h_{2}$ are quasi-isometric and write $h_{1} \sim h_{2}$ if there are positive constants $c$ and $C$ such that $c h_{1} \leq h_{2} \leq C h_{1}$. Similarly, if $\omega_{1}$ and $\omega_{2}$ are $(1,1)$-forms or functions, and there exist positive constants $c$ and $C$ such that $c \omega_{1} \leq \omega_{2} \leq C \omega_{1}$, we write $\omega_{1} \sim \omega_{2}$. If $\omega_{1}$ and $\omega_{2}$ are positive $(1,1)$-forms and $\omega_{1} \sim \omega_{2}$, we say that $\omega_{1}$ and $\omega_{2}$ are quasi-isometric. If $\omega_{1} \geq \omega_{2}$ and $\omega_{2} \sim \omega_{3}$ we write $\omega_{1} \tilde{\geq} \omega_{3}$. When a function $f_{1}$ is dominated by a constant multiple of a function $f_{2}$ near the exceptional divisor we will write $f_{1}=O\left(f_{2}\right)$.

REMARK (1.3.1). All metrics on a compact manifold are quasiisometric. If $\omega_{1}$ and $\omega_{2}$ are positive $C^{\infty}(1,1)$-forms on an open neighbourhood of a point $q$, then $\omega_{1}$ and $\omega_{2}$ are quasi-isometric in a neighbourhood of $q$. In local coordinates $\left(z_{1}, \ldots, z_{n}\right)$ near $q$, every positive $C^{\infty}(1,1)$-form is locally quasi-isometric to the Euclidean form

$$
\omega_{\text {Eucl }}=\frac{\sqrt{-1}}{\pi} \sum_{i=1}^{n} d z_{i} \wedge d \bar{z}_{i}
$$


(1.4) Poincaré metrics. Recall that the fundamental form of the Poincaré metric on the punctured disc $\Delta^{*}=\{z \in \mathbf{C}: 0<|z|<1\}$ is

$$
\begin{aligned}
\omega_{\Delta^{*}} & =\frac{\sqrt{-1}}{\pi} \frac{d z \wedge d \bar{z}}{|z|^{2}\left(\log |z|^{2}\right)^{2}} \\
& =-\frac{\sqrt{-1}}{2 \pi} \partial \bar{\partial} \log \left(\log |z|^{2}\right)^{2} .
\end{aligned}
$$

We are interested only in the asymptotic behaviour of $\omega_{\Delta^{*}}$ near the puncture in $\Delta^{*}$.

More generally, let $M$ be a compact complex manifold and $D$ a divisor with normal crossings. If $q$ is a point of $D$ at which $k$ components intersect, then $q$ has a neighbourhood whose intersection with $M-D$ is of the form $\left(\Delta^{*}\right)^{k} \times \Delta^{n-k}$. A metric on $M-D$ is said to be Poincaré if it is quasi-isometric near each such $q$ to a product of Poincaré metrics on $\left(\Delta^{*}\right)^{k}$ and Euclidean metrics on $\Delta^{n-k}$. It is always possible to construct a Poincaré metric on $M-D$ by patching together local forms with $C^{\infty}$ partitions of unity. If $M$ is Kähler, it is easy to construct Kähler Poincaré metrics on $M-D$ by a global construction (see $\S 5.4$ ).

(1.5) Normal coordinates. Let $M$ and $D$ be as above, let $E_{1}, \ldots, E_{k}$ be the components of $D$ passing through a point $q$, and let $\left(z_{1}, \ldots, z_{n}\right)$ be local coordinates on a neighbourhood $U$ of $q$ such that no other component of $D$ intersects $\bar{U}$. We call the coordinates $\left(z_{1}, \ldots, z_{n}\right)$ normal coordinates for $E_{1}, \ldots, E_{k}$ if $E_{i}$ is given locally near $q$ by the equation $z_{i}=0$ for $1 \leq i \leq k$.

The fundamental form $\omega_{\text {Poinc }}$ of a Poincaré metric on $M-D$ may be described locally in any system of normal coordinates by the quasi-isometry

$$
\omega_{\text {Poinc }} \sim \frac{\sqrt{-1}}{\pi}\left(\sum_{i=1}^{k} \frac{d z_{i} \wedge d \bar{z}_{i}}{\left|z_{i}\right|^{2}\left(\log \left|z_{i}\right|^{2}\right)^{2}}+\sum_{i=k+1}^{n} d z_{i} \wedge d \bar{z}_{i}\right)
$$

$$
\sim \frac{\sqrt{-1}}{\pi}\left(\sum_{i=1}^{k} \frac{d z_{i} \wedge d \bar{z}_{i}}{\left|z_{i}\right|^{2}\left(\log \left|z_{i}\right|^{2}\right)^{2}}+\sum_{i=1}^{n} d z_{i} \wedge d \bar{z}_{i}\right) .
$$

Note that we are concerned only with the asymptotic behaviour of these forms near $z_{1} z_{2} \ldots z_{k}=0$. We may assume that $U$ is small 
enough that singularities of the forms on the right at $\left|z_{i}\right|=1$ may be ignored.

(1.6) Modified Poincaré metrics. We call a metric on $M-D$ a modified Poincaré metric if its fundamental form $\omega_{P}$ may be described locally in normal coordinates by the quasi-isometry

$$
\omega_{P} \sim \sum_{i=1}^{m} \tau_{i}^{*} \omega_{\Delta^{*}}+\frac{\sqrt{-1}}{\pi} \sum_{i=1}^{n} d z_{i} \wedge d \bar{z}_{i}
$$

where each $\tau_{i}$ is a nonconstant monomial map from $\left(\Delta^{*}\right)^{k} \times \Delta^{n-k}$ to $\Delta^{*}$ of the form $\tau_{i}\left(z_{1}, \ldots, z_{n}\right)=z_{1}^{\lambda_{i 1}} z_{2}^{\lambda_{i 2}} \ldots z_{k}^{\lambda_{i k}}$ and the $m \times k$ matrix $\Lambda=\left(\lambda_{i j}\right)$ has nonnegative integer entries and at least one positive entry in each row and column. We note that there may be many different non-quasi-isometric modified Poincaré metrics on $M-D$. We show in Corollary (5.2.6) that the description (1.6.1) is independent of the choice of normal coordinates. The matrix $\left(\lambda_{i j}\right)$ determines the local quasi-isometry class of the metric, but not vice-versa.

We will say that a modified Poincaré metric is a homogeneous Poincaré metric if its fundamental form $\omega_{P, \text { hom }}$ may be described locally in normal coordinates by the quasi-isometry

$$
\omega_{P, \text { hom }} \sim \frac{\sqrt{-1}}{\pi}\left(\frac{1}{\left(\log \left|z_{1} z_{2} \ldots z_{k}\right|^{2}\right)^{2}} \sum_{i=1}^{k} \frac{d z_{i} \wedge d \bar{z}_{i}}{\left|z_{i}\right|^{2}}+\sum_{i=1}^{n} d z_{i} \wedge d \bar{z}_{i}\right) .
$$

This description is also independent of the choice of normal coordinates (Corollary (5.3.5)).

A little linear algebra shows that a modified Poincaré metric is homogeneous if the matrix $\Lambda$ has rank $k$ and all the entries of $\Lambda$ are positive (Lemma (5.3.3)). If the matrix $\Lambda$ can be chosen to be the identity matrix of suitable dimensions, the modified Poincaré metric is Poincaré. We construct Kähler modified Poincaré metrics and Kähler homogeneous Poincaré metrics in $§ 5.4$.

(1.7) Saper's distinguished metrics. Saper [S2] calls a metric on $M-D$ distinguished if its fundamental form $\omega_{\text {Sap }}$ may be described locally in normal coordinates by the quasi-isometry 
(1.7.1) $\omega_{\text {Sap }} \sim \frac{\sqrt{-1}}{\pi}\left(\frac{1}{\left(\log \left|z_{1} z_{2} \ldots z_{k}\right|^{2}\right)^{2}} \sum_{i=1}^{k} \frac{d z_{i} \wedge d \bar{z}_{i}}{\left|z_{i}\right|^{2}}\right.$

$$
\left.+\frac{1}{\left.|\log | z_{1} z_{2} \ldots z_{k}\right|^{2} \mid} \sum_{i=1}^{n} d z_{i} \wedge d \bar{z}_{i}\right) \text {. }
$$

This description is independent of the choice of normal coordinates (Corollary (5.3.5)). We will usually refer to such a metric simply as a Saper metric.

If $\omega$ is the fundamental form of a metric on the compact manifold $M$, and $\omega_{\text {Sap }}$ and $\omega_{P, \text { hom }}$ are, respectively, fundamental forms of Saper and homogeneous Poincaré metrics on the noncompact manifold $M-D$, then

$$
\omega_{\text {Sap }}+\omega \sim \omega_{P, \text { hom }}
$$

(1.8) Modified Saper metrics. Let $M$ and $\tilde{M}$ be compact complex manifolds, let $D$ be an effective divisor on $\tilde{M}$ with normal crossings, and let $\pi: \tilde{M} \rightarrow M$ be a holomorphic map such that the restriction of $\pi$ to $\tilde{M}-D$ is a biholomorphism onto its image. The divisor $D$ may be expressed as $D=\sum_{i=1}^{m} \lambda_{i} E_{i}$, where $E_{1}, E_{2}, \ldots, E_{m}$ are smooth, reduced, irreducible divisors on $\tilde{M}$ which simultaneously have only normal crossings, and $\lambda_{1}, \lambda_{2}, \ldots, \lambda_{m}$ are positive integers. Let $L=[D]$ be the line bundle on $\tilde{M}$ associated with $D$ and let $h$ be a hermitian metric on $L$. Let $s: \tilde{M} \rightarrow L$ be a global holomorphic section of $L$ such that $(s)=D$. Such a section always exists since $D$ is effective (see $\S 3.4$ ). We denote by $\|s\|$ the norm of $s$ under the metric $h$. Since $\tilde{M}$ is compact, we may also choose $s$ so that $\|s\|<1$ everywhere on $\tilde{M}$. The Chern form of $L$ with respect to the metric $h$ may be written as

$$
c_{1}(L, h)=-\frac{\sqrt{-1}}{2 \pi} \partial \bar{\partial} \log \|s\|^{2}
$$

(cf. §3.5). We define on $\tilde{M}-D$ a Poincaré-type $(1,1)$-form $\nu$ associated with the divisor $D$, the section $s$, and the metric $h$ by

$$
\nu=-\frac{\sqrt{-1}}{2 \pi} \partial \bar{\partial} \log \left(\log \|s\|^{2}\right)^{2} .
$$


The crucial property of the Poincaré-type form $\nu$ is that it splits as $\nu=\mu+\eta$ where

$$
\mu=\frac{2}{\log \|s\|^{2}} c_{1}(L, h)
$$

and the form $\eta$ near $D$ is essentially the pullback of the Poincare form $\omega_{\Delta^{*}}$ under a monomial map, plus low order terms. More precisely, suppose that $z_{1}, \ldots, z_{n}$ are normal coordinates for the components of $D$ in a neighbourhood of a point $p \in D$ such that $D$ is given locally by the vanishing of the monomial

$$
\tau(z)=z_{1}^{\lambda_{1}} z_{2}^{\lambda_{2}} \ldots z_{k}^{\lambda_{k}} .
$$

We treat $\tau$ as a map from a neighbourhood $U$ of $p$ to the disc $\Delta=\{z \in \mathbf{C}:|z|<1\}$. Let $\omega_{\text {Eucl }}$ be the local Euclidean $(1,1)$-form

$$
\omega_{\mathrm{Eucl}}=\frac{\sqrt{-1}}{\pi} \sum_{i=1}^{n} d z_{i} \wedge d \bar{z}_{i}
$$

and let $\beta=-\log \|s\|^{2}$. We will show in $\S 5$ that

$$
\frac{1}{\beta} \omega_{\mathrm{Eucl}}+\eta \sim \frac{1}{\beta} \omega_{\mathrm{Eucl}}+\tau^{*} \omega_{\Delta^{*}}
$$

near $p$. Note that $\beta \neq 0$ on $\tilde{M}-D$ and $\beta \rightarrow \infty$ as we approach $D$. Let $D_{1}, \ldots, D_{r}$ be divisors of the form

$$
D_{i}=\sum_{j=1}^{m} b_{i j} E_{j}
$$

such that the matrix $\left(b_{i j}\right)$ has nonnegative integer entries and at least one positive entry in each row and column, i.e. the divisors $D_{i}$ are effective and their sum has the same support as $D$. We may choose sections $s_{i}$ and metrics $h_{i}$ for the line bundles $\left[D_{i}\right]$ as above, and use them to construct Poincaré-type forms $\nu_{i}$. Let $\omega$ be the fundamental form of a hermitian metric on $M$ and let $l_{0}, l_{1}, \ldots, l_{r}$ be positive integers.

Definition (1.8.3). We will call any expression of the form

$$
\omega_{S}=l_{0} \pi^{*} \omega+\sum_{i=1}^{r} l_{i} \nu_{i}
$$


a modified Saper form which is distinguished with respect to $\pi$. We will call a metric on $\tilde{M}-D$ a modified Saper metric if its fundamental form is quasi-isometric to a modified Saper form.

If $Y$ is a smooth submanifold of $\tilde{M}$ which has normal crossings with $D$, we also call the induced metric on $Y-(Y \cap D)$ a modified Saper metric. We are particularly interested in the case in which the closure $X$ of $\pi(Y-(Y \cap D))$ in $M$ is singular, so that $Y$ is an embedded resolution of a singular analytic subspace $X \subset M$.

Every positive modified Saper form $\omega_{S}$ determines a modified Saper metric. If in addition $\omega$ is Kähler, then so is $\omega_{S}$. We will show that the sum of a modified Saper metric on $\tilde{M}-D$ and the restriction of a metric on $\tilde{M}$ is a modified Poincaré metric on $\tilde{M}-D$.

As with modified Poincaré metrics, there may be many different non-quasi-isometric modified Saper metrics on $\tilde{M}-D$.

Let $\omega_{\text {Sap }}$ be the fundamental form of a distinguished Saper metric on $\tilde{M}-D$, as defined in $\S 1.7$.

DEFINITION (1.8.4). We will say that a modified Saper metric and its fundamental form $\omega_{S \text {,hom }}$ are homogeneous if $\omega_{S \text {,hom satisfies }}$

$$
\omega_{S, \text { hom }} \sim \pi^{*} \omega+\omega_{\text {Sap }}
$$

The sum of a homogeneous Saper metric on $\tilde{M}-D$ and the restriction of a metric on $\tilde{M}$ is a homogeneous Poincaré metric on $\tilde{M}-D$.

\section{(1.9) $L_{2}$-cohomology of Poincaré and Saper metrics. Let $X$} be a reduced compact analytic subspace of a compact Kähler manifold $M$. Let $\pi: \tilde{M} \rightarrow M$ be a composite of blow-ups of the type described in $\S 1.1$ which resolves the singularities of $X$, let $\tilde{X}$ be the strict transform of $X$ in $\tilde{M}$, and let $D$ be the exceptional divisor of $\pi$ in $\tilde{M}$. Then $\tilde{X}$ is smooth and has normal crossings with $D$, and

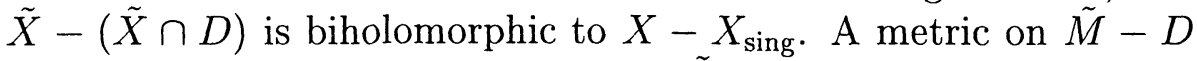

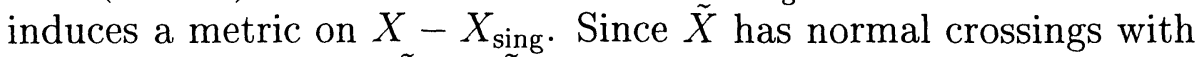
$D$, the restriction to $\tilde{X}-(\tilde{X} \cap D)$ of a distinguished Saper metric on $\tilde{M}-D$ is a distinguished Saper metric on $\tilde{X}-(\tilde{X} \cap D)$, and similarly for Poincaré, modified Poincaré, homogeneous Poincaré, modified Saper, and homogeneous Saper metrics.

It is always possible to construct distinguished metrics on $\tilde{M}-D$ using $C^{\infty}$ partitions of unity, but these metrics may not be Kähler. 
Saper [S2] constructed Kähler distinguished metrics in the case that $X$ is a compact Kähler variety with isolated singularities, and used Goresky-MacPherson's characterization of intersection cohomology to prove that the $L_{2}$-cohomology of $X$ with respect to such a metric is isomorphic to the intersection cohomology of $X$.

In contrast, the $L_{2}$-cohomology of $\tilde{X}$ with respect to a Poincaré metric on $\tilde{X}-(\tilde{X} \cap D)$ is isomorphic to the cohomology of $\tilde{X}([\mathbf{Z 1}]$, [Z2]). It is easy to construct a Kähler Poincaré metric on $\tilde{M}-D$ and hence on $\tilde{X}-(\tilde{X} \cap D)$ (see $\S 5.4)$.

Although we have restricted ourselves in this paper to subvarieties of compact Kähler manifolds, similar constructions can be done in more general situations. In particular, if $X$ is any compact Kähler variety with isolated singularities, we may make use of local embeddings of neighbourhoods of the singular points of $X$ into domains in $\mathbf{C}^{N}$, to construct our metrics on $X-X_{\text {sing. }}$. Our modified Saper metrics on varieties with isolated singularities are exactly Saper distinguished metrics.

Let $h$ be a hermitian metric on $X-X_{\text {sing }} \cong \tilde{X}-(\tilde{X} \cap D)$. We define associated complexes of $L_{2}$ sheaves $\mathcal{S}$ on $X$ and $\tilde{\mathcal{S}}$ on $\tilde{X}$ as follows. Let $\mathcal{S}_{0}$ be the complex of presheaves on $X$ whose sections over any open set $U$ in $X$ are smooth measurable differential forms $\phi$ on $U \cap\left(X-X_{\text {sing }}\right)$ such that both $\phi$ and $d \phi$ are $L_{2}$-bounded with respect to $h$. Let $\mathcal{S}$ be the associated complex of sheaves on $X$. Similarly, we define a complex of presheaves $\tilde{\mathcal{S}}_{0}$ on $\tilde{X}$, whose sections over any open set $U$ in $\tilde{X}$ are smooth measurable differential forms $\phi$ on $U-(U \cap D)$ such that both $\phi$ and $d \phi$ are $L_{2}$-bounded with respect to $h$, and let $\tilde{\mathcal{S}}$ be the associated complex of sheaves on $\tilde{X}$. One of the conditions needed to apply Goresky-MacPherson's theorem to $\mathcal{S}$ is that $\mathcal{S}$ must be a complex of fine sheaves. We will show that if $h$ is a modified Saper or modified Poincaré metric then $\mathcal{S}$ on $X$ is fine, and that if $h$ is a modified Poincaré metric then $\tilde{\mathcal{S}}$ on $\tilde{X}$ is also fine. We prove corresponding statements for $L_{2}$ sheaves on $M$ and $\tilde{M}$.

\section{§2. Main results and guidance through the paper.}

(2.1) Theorem I. Our first main result concerns the existence of complete Kähler modified Saper metrics. We prove parts (i) and (ii) in $\S 8$, part (iii) in $\S 9$, and part (iv) in $\S 10$. 
THEOREM I. Let $M_{0}$ be a compact Kähler manifold with Kähler form $\omega_{0}$. Suppose that $\left\{\pi_{j}: M_{j} \rightarrow M_{j-1}\right\}$ is a finite sequence of blow-ups along smooth centres $C_{j} \subset M_{j-1}$, chosen so that $C_{j}$ has normal crossings with the total exceptional divisor $D_{j-1}$ of the composite of the first $j-1$ blow-ups. Then

i. There exist complete Kähler modified Saper metrics with fundamental forms $\omega_{S, j}$, constructed inductively on the manifolds $M_{j}-D_{j}$. These metrics are bounded below by the sum of a Saper distinguished metric and an induced metric from $M_{0}$, and are quasi-isometric to this lower bound in the case that for $2 \leq i \leq j$ either $C_{i} \subset D_{i-1}$ or $C_{i}$ is disjoint from $D_{i-1}$. If, in addition, $\operatorname{dim} C_{1}=0$ and $\operatorname{dim} C_{i}=0$ for each $i$ such that $C_{i}$ is disjoint from $D_{i-1}$ (i.e. the image of $D_{j}$ in $M_{0}$ consists of isolated points in $M_{0}$ ), these metrics are exactly Saper distinguished metrics.

ii. The sum of a modified Saper metric on $M_{j}-D_{j}$ and the restriction of a metric on $M_{j}$ is a modified Poincaré metric on $M_{j}-D_{j}$, which in turn is bounded above by a true Poincaré metric.

iii. There also exist complete Kähler homogeneous Saper metrics on $M_{j}-D_{j}$.

iv. The complex of $L_{2}$ sheaves on $M_{0}$ associated with any modified Saper or modified Poincaré metric on $M_{j}-D_{j}$ is fine. The complex of $L_{2}$ sheaves on $M_{j}$ associated with any modified Poincaré metric on $M_{j}-D_{j}$ is also fine.

The bounds of (i) and (ii) are written more concisely in terms of the composite $\pi_{j, 0}$ of the first $j$ blow-ups and the fundamental forms $\omega_{j}$ of a metric on $M_{j}, \omega_{S, j}$ and $\omega_{P, j}$ of the modified Saper and modified Poincaré metrics, $\omega_{\text {Sap }}$ of a Saper distinguished metric, and $\omega_{\text {Poinc }}$ of a true Poincaré metric on $M_{j}-D_{j}$ :

$$
\pi_{j, 0}^{*} \omega_{0}+\omega_{\text {Sap }} \stackrel{\tilde{\leq}}{\leq} \omega_{S, j}<\omega_{S, j}+\omega_{j}=\omega_{P, j} \stackrel{\tilde{\leq}}{\leq} \omega_{\text {Poinc }} .
$$

The fundamental form $\omega_{S}$ of the metric in (iii) satisfies the quasiisometry $\omega_{S} \sim \pi_{j, 0}^{*} \omega_{0}+\omega_{\text {Sap }}$.

Suppose that $X$ is a reduced compact analytic subspace of $M_{0}$ and the maps $\pi_{j}$ determine a desingularization of $X$ of the type described in $\S 1$. Let $\pi: \tilde{M} \rightarrow M_{0}$ be the composite of all the 
blow-ups, let $D$ be the exceptional divisor of $\pi$, and let $\tilde{X}$ be the (smooth) strict transform of $X$ in $\tilde{M}$. Since $\tilde{X}$ and $D$ have normal crossings, the metrics we construct on $\tilde{M}-D$ induce metrics with the corresponding properties on $\tilde{X}-(\tilde{X} \cap D) \cong X-X_{\text {sing }}$.

(2.2) Theorem II. Our second main theorem states that there is a natural relationship between incomplete metrics which determine an embedded resolution of singularities, and complete modified Saper and modified Poincaré metrics.

Definition (2.2.1). Let $X$ be a reduced compact analytic subspace of a compact Kähler manifold $M$. Let $h$ be an incomplete hermitian metric on $M^{\prime}=M-X_{\text {sing. }}$. We will say that $h$ determines an embedded resolution of the singularities of $X$ if the following conditions hold:

i. The completion $\tilde{M}$ of $M^{\prime}$ under $h$ is a compact Kähler manifold and the completion $\tilde{X}$ of $X$ in $\tilde{M}$ is also smooth.

ii. If $\iota: M^{\prime} \hookrightarrow \tilde{M}$ is the natural embedding of $M^{\prime}$ into its completion then $\tilde{M}-\iota\left(M^{\prime}\right)$ is the support of an effective divisor $D$ on $\tilde{M}$ with only normal crossings, and the divisor $D$ has only normal crossings with $\tilde{X}$.

iii. The map $\iota^{-1}: \tilde{M}-D \rightarrow M^{\prime}$ extends to a holomorphic map

$$
\pi: \tilde{M} \rightarrow M
$$

which is a biholomorphism from $\tilde{M}-D$ to $M^{\prime}=M-X_{\text {sing }}$.

THEOREM II. Let $X$ be a reduced compact analytic subspace of a compact Kähler manifold $M$ and let $\omega$ be a Kähler form on $M$. Suppose that $f$ is a positive $C^{\infty}$ function on $M-X_{\text {sing }}$ such that $f<1$ and such that for all sufficiently large integers $l$, the $(1,1)$ form

$$
\tilde{\omega}=l \omega+\frac{\sqrt{-1}}{2 \pi} \partial \bar{\partial} \log f
$$

is the Kähler form of an incomplete metric on $M-X_{\text {sing }}$ which determines an embedded resolution $\tilde{X} \hookrightarrow \tilde{M}$ of the singularities of $X$. Let $D$ be the associated divisor of the map $\pi: \tilde{M} \rightarrow M$ described above and let $s$ be a section of the line bundle $[D]$ such that the divisor $(s)$ determined by $s$ is $D$. Suppose also that the function $\pi^{*} f$ on $\tilde{M}-D$ extends to the norm-squared $\|s\|^{2}$ of $s$ on 
$\tilde{M}$ under some hermitian metric on $[D]$. Then for all sufficiently large integers $l$, the $(1,1)$-form

$$
\omega_{S}=l \omega-\frac{\sqrt{-1}}{2 \pi} \partial \bar{\partial} \log (\log f)^{2}
$$

on $M-X_{\text {sing }}$ is the Kähler form of a complete modified Saper metric on $M-X_{\text {sing }} \cong \tilde{M}-D$. Moreover the associated complexes of $L_{2}$ sheaves on $M$ and $X$ are fine.

We construct Kähler forms $\tilde{\omega}$ with the required property in $\S 4$. The proof of Theorem II is given in $\S 9.2$ (Theorem (9.2.1)) and $\S 10$.

REMARK. In practice, the generating function $f$ for the Poincarétype $(1,1)$-form $-\frac{\sqrt{-1}}{2 \pi} \partial \bar{\partial} \log (\log f)^{2}$ may be very constructive in nature, reflecting the blow-ups used to resolve $X$. There is a procedure for constructing such a function $f$ explicitly, locally near $p \in X_{\text {sing }}$, as an expression of the form $\sum_{i=1}^{r}\left|w_{i}\right|^{2}$, where $w_{i}$ is a holomorphic function on a neighbourhood $U$ of $p$ which vanishes on $U \cap X_{\text {sing }}$. An algorithm for this construction is not included in this paper but will be given elsewhere.

(2.3) Guidance through the paper. This paper is organized as follows. In $\S 3$ we introduce local coordinates for the blow-up $\pi$ : $\tilde{M} \rightarrow M$ of a complex manifold $M$ along a smooth centre $C$. We use these coordinates to describe the exceptional divisor $E=\pi^{-1}(C)$ and the associated line bundle $L=[E]$. If $s: \tilde{M} \rightarrow L$ is a nonzero global holomorphic section of $L$ and $h$ is a hermitian metric on $L$, then the Chern form of the metric $h$ on $L$ is the $(1,1)$-form

$$
c_{1}(L, h)=-\frac{\sqrt{-1}}{2 \pi} \partial \bar{\partial} \log \|s\|^{2} .
$$

We may always choose the section $s$ so that its associated divisor $(s)$ is $E$. We conclude $\S 3$ with some linear algebra lemmas on the accumulation of exceptional divisors under repeated blow-ups. These lemmas will be used in $\S 8$ in estimating the asymptotic rates of growth of our metrics near the singular locus of $X$.

In $\S 4$ we use the tubular neighbourhood construction of the appendix to show that if $\omega$ is a Kähler form on $M$, then for a suitable metric $h$ on $L$, the $(1,1)$-form

$$
\tilde{\omega}=l \pi^{*} \omega-c_{1}(L, h)
$$


is the fundamental form of a Kähler metric on $\tilde{M}$ for all sufficiently large integers $l$ (Theorem (4.2.2)). We construct metrics inductively on repeated blow-ups in this way.

In $\S 5$ we study Poincaré-type $(1,1)$-forms on the complement of an effective divisor $D$ with normal crossings in a compact complex manifold $M$. Given a hermitian metric $h$ on the line bundle $L=[D]$ and a global holomorphic section $s$ of $L$ such that $(s)=D$ and $\|s\|<1$ on $M$, we construct a Poincaré-type $(1,1)$-form

$$
\nu=-\frac{\sqrt{-1}}{2 \pi} \partial \bar{\partial} \log \left(\log \|s\|^{2}\right)^{2}
$$

The form $\nu$ splits into two essential parts $\nu=\mu+\eta$ where

$$
\mu=\frac{2}{\log \|s\|^{2}} c_{1}(L, h)
$$

and where, in local normal coordinates near $D$, the form $\eta$ is the pullback of the Poincaré form $\omega_{\Delta^{*}}$ under a monomial map $\tau$ associated with $D$, plus low order terms. If $p$ is a point in $D$ at which $k$ components of $D$ intersect, and if $z_{1}, \ldots, z_{n}$ are local normal coordinates in which the components of $D$ through $p$ are given by the equations $z_{i}=0$ for $1 \leq i \leq k$, then the monomial map $\tau$ is given by

$$
\tau\left(z_{1}, \ldots, z_{n}\right)=z_{1}^{\lambda_{1}} z_{2}^{\lambda_{2}} \ldots z_{k}^{\lambda_{k}}
$$

where $\lambda_{i}$ is the multiplicity of the $i$ th component of $D$ through $p$.

This splitting leads to the crucial idea in our construction of modified Saper metrics: if we choose appropriate divisors $D_{j}$ and corresponding Poincaré-type forms $\nu_{j}$, we can use the forms $\mu_{j}$ to obtain the positivity we need and the forms $\eta_{j}$ to get completeness. Using this splitting, it is easy to construct Poincaré and modified Poincaré metrics (Proposition (5.4.1)). We also show that the sum of a modified Saper metric on $M-D$ and a Kähler metric on $M$ is always a modified Poincaré metric on $M-D$ (Proposition (5.5.1)).

In $\S 6$ we show that whenever a metric on $M-D$ is bounded below, locally near each point of $D$, by the pullback of the Poincaré form $\omega_{\Delta^{*}}$ under a monomial map $\tau$ associated with $D$, then the metric is complete.

In $\S 7$ we introduce our complete metrics for a single blow-up $\pi$ : $\tilde{M} \rightarrow M$ of a compact Kähler manifold $M$ along a submanifold 
$C \subset M$. We start with a Kähler form $\omega$ on $M$ and choose a metric $h$ on the line bundle $L$ associated with $\pi$, such that the $(1,1)$-form $\tilde{\omega}=l \pi^{*} \omega-c_{1}(L, h)$ of (2.3.1) above is the fundamental form of a Kähler metric on $\tilde{M}$ for sufficiently large $l$. Using an appropriate section $s$ of $L$, we construct a Poincaré-type (1,1)-form $\nu$, as in (2.3.2) above, on the complement of the exceptional divisor $E=$ $\pi^{-1}(C)$ in $\tilde{M}$. The fundamental form of our modified Saper metric on $\tilde{M}-E$ is

$$
\omega_{S}=l \pi^{*} \omega+\nu
$$

where $l$ is a sufficiently large positive integer. The $(1,1)$-form

$$
\omega_{P}=\omega_{S}+\tilde{\omega}
$$

is the fundamental form of a Poincaré metric on $\tilde{M}-E$. In Proposition (7.2.1) we use the results of $\S 5$ to give the precise asymptotic behaviour of our metrics near the exceptional divisor $E$. If $\left(z_{1}, \ldots, z_{n}\right)$ are normal coordinates near a point in $E$, such that $E$ is given locally by the equation $z_{1}=0$, then locally

$\omega_{S} \sim \pi^{*} \omega+\frac{\sqrt{-1}}{\pi}\left(\frac{1}{\left.|\log | z_{1}\right|^{2} \mid} \sum_{i=1}^{n} d z_{i} \wedge d \bar{z}_{i}+\frac{d z_{1} \wedge d \bar{z}_{1}}{\left|z_{1}\right|^{2}\left(\log \left|z_{1}\right|^{2}\right)^{2}}\right)$,

i.e. our modified Saper metric for one blow-up is locally quasiisometric to the sum of the metric induced from $M$ and a Saper distinguished metric. Suppose that $z_{2}, \ldots, z_{k}$ are fibre coordinates for the map $E \rightarrow C$. Then locally

$$
\begin{aligned}
\omega_{S} \sim \frac{\sqrt{-1}}{\pi} & \left(\frac{d z_{1} \wedge d \bar{z}_{1}}{\left|z_{1}\right|^{2}\left(\log \left|z_{1}\right|^{2}\right)^{2}}\right. \\
& \left.\quad+\frac{1}{\left.|\log | z_{1}\right|^{2} \mid} \sum_{i=2}^{k} d z_{i} \wedge d \bar{z}_{i}+\sum_{i=k+1}^{n} d z_{i} \wedge d \bar{z}_{i}\right) .
\end{aligned}
$$

These results are generalized in $\S 8$ and $\S 9$.

In $\S 8$ we introduce our complete metrics $\omega_{S, j}$ inductively for a sequence of blow-ups and prove parts (i) and (ii) of Theorem I. Let $\left\{\pi_{j}: M_{j} \rightarrow M_{j-1}\right\}$ be a sequence of blow-ups along smooth centres $C_{j}$ of the type described in $\S 1.1$. We take as $\omega_{S, 1}$ the modified Saper 
form $\omega_{S}$ of $\S 7$. If $\omega_{S, j-1}$ is the modified Saper form obtained after $j-1$ blow-ups, we take as $\omega_{S, j}$ the $(1,1)$-form

$$
\omega_{S, j}=l \pi_{j}^{*} \omega_{S, j-1}+\nu_{j}
$$

where $l$ is a sufficiently large positive integer and $\nu_{j}$ is a Poincarétype $(1,1)$-form associated with the total exceptional divisor $D_{j}$ of the composite of the first $j$ blow-ups. In Theorem (8.4.1), we use the results of $\S 3, \S 5$, and $\S 7$ to obtain the asymptotic behaviour of our modified Saper metrics near any point $p$ in $D_{j}$. We may choose local normal coordinates $\left(z_{1}, \ldots, z_{n}\right)$ for $M_{j}$ in which $D_{j}$ is given locally by the equation $z_{1}^{\lambda_{1}} z_{2}^{\lambda_{2}} \ldots z_{k}^{\lambda_{k}}=0$ for some positive integers $\lambda_{1}, \ldots, \lambda_{k}$. We let $\tau_{j}$ be the corresponding monomial map to the punctured disc $\Delta^{*}$, given by

$$
\tau_{j}\left(z_{1}, \ldots, z_{n}\right)=z_{1}^{\lambda_{1}} z_{2}^{\lambda_{2}} \ldots z_{k}^{\lambda_{k}} .
$$

We may also assume that the map from the exceptional divisor $E_{j}=\pi_{j}^{-1}\left(C_{j}\right)$ to the $j$ th centre $C_{j}$ has fibre coordinates $\left\{z_{i}\right\}_{i \in I}$ for some subset $I$ of $\{1, \ldots, n\}$. We let $d z_{f} \wedge d \bar{z}_{f}=\sum_{i \in I} d z_{i} \wedge d \bar{z}_{i}$. In terms of such coordinates, the asymptotic behaviour of $\omega_{S, j}$ is given locally by

$$
\omega_{S, j} \sim \pi_{j}^{*} \omega_{S, j-1}+\frac{\sqrt{-1}}{\pi}\left(\frac{1}{\left.|\log | z_{1} z_{2} \ldots z_{k}\right|^{2} \mid} d z_{f} \wedge d \bar{z}_{f}\right)+\tau_{j}^{*} \omega_{\Delta^{*}} .
$$

The last two terms correspond to the terms $\mu_{j}$ and $\eta_{j}$ in the decomposition of the Poincaré-type form $\nu_{j}$. This asymptotic behaviour is one of the key points of our results. The monomial map $\tau_{j}$ is closely related to the linear algebra of the accumulation of exceptional divisors given in $\S 3$. The powers $\lambda_{i}$ occurring in the map $\tau_{j}$ are the multiplicities of the components of the total exceptional divisor $D_{j}$ passing through $p$.

We construct homogeneous Saper metrics in $\S 9$ and prove Theorem I (iii) and Theorem II, except for the statement about fine sheaves. In $\S 10$ we complete the proofs of Theorems I and II by proving the results on fine sheaves.

\section{$\S 3$. Background on geometry of blow-ups.}

(3.1) Local coordinates for blow-ups. Let $M$ be a compact complex manifold of dimension $n$, let $C$ be a submanifold of codimension $k>1$, and let 


$$
\pi: \tilde{M} \rightarrow M
$$

be the blow-up of $M$ along the centre $C$. The blow-up can be described in local coordinates as follows. Let $p$ be a point in $C$ and let $V$ be a coordinate neighbourhood of $p$ in $M$, centered at $p$, with holomorphic coordinates $\left(Z_{1}, Z_{2}, \ldots, Z_{n}\right)$ such that

i. $C \cap V$ is given by $Z_{1}=Z_{2}=\ldots=Z_{k}=0$ and

ii. $Z_{k+1}, \ldots, Z_{n}$ are local coordinates on $C$.

Let $\xi_{1}, \xi_{2}, \ldots, \xi_{k}$ be homogeneous coordinates on $\mathbf{P}^{k-1}$. The set $\pi^{-1}(V)$ in $\tilde{M}$ is biholomorphic to the subset $\tilde{V}$ of $V \times \mathbf{P}^{k-1}$ given by

$$
\tilde{V}=\left\{(Z,[\xi]): Z_{i} \xi_{j}=Z_{j} \xi_{i}, \quad 1 \leq i, j \leq k\right\} .
$$

We cover $\tilde{V}$ by open sets $U_{\jmath}=\left\{\xi_{j} \neq 0\right\}$, for $j=1,2, \ldots, k$, with coordinates $\left(z_{j 1}, \ldots, z_{j n}\right)$, where

$$
\begin{array}{ll}
z_{j i}=\frac{\xi_{i}}{\xi_{j}}=\frac{Z_{i}}{Z_{j}}, & \text { if } \quad 1 \leq i \leq k \text { and } i \neq j \\
z_{j l}=Z_{l} & \text { if } l=j \text { or } k+1 \leq l \leq n .
\end{array}
$$

The restriction of $\pi$ to $U_{j}$ is given by the equations

$$
\begin{array}{ll}
Z_{i}=z_{j j} z_{j i} & \text { if } \quad 1 \leq i \leq k \quad \text { and } \quad i \neq j \\
Z_{l}=z_{j l} & \text { if } l=j \text { or } k+1 \leq l \leq n .
\end{array}
$$

We will sometimes find it convenient to refer separately to $C$ coordinates and the remaining coordinates in the directions normal to $C$, setting

$$
Z_{N}=\left(Z_{1}, \ldots, Z_{k}\right) \quad \text { and } \quad Z_{C}=\left(Z_{k+1}, \ldots, Z_{n}\right) .
$$

Similarly,

$$
z_{j N}=\left(z_{j 1}, \ldots, z_{j k}\right) \quad \text { and } \quad z_{C}=\left(z_{j, k+1}, \ldots, z_{j n}\right) .
$$

If $q$ is any point in $\pi^{-1}(p)$ and if $1 \leq j \leq k$, then by a suitable choice of homogeneous coordinates on $\pi^{-1}(p) \cong \mathbf{P}^{k-1}$, corresponding to a linear change in the coordinates $Z_{1}, \ldots, Z_{k}$, we may assume that $q$ is the origin, given by $z_{j 1}=\ldots=z_{j n}=0$, in the open set $U_{j}$. 
(3.2) The exceptional divisor E. The exceptional divisor of the blow-up is $E=\pi^{-1}(C)$. In the open set $U_{j}$ in $\tilde{M}, E$ is given by $z_{j j}=0$. The map $\pi$ is a biholomorphism of $\tilde{M}-E$ onto $M-C$ and maps $E$ onto $C$. Let $\tau: E \rightarrow C$ be the restriction of $\pi$ to $E$. In local coordinates on $U_{j}$ and $C$ we have

$$
\tau\left(z_{j 1}, \ldots, z_{j n}\right)=\left(z_{j, k+1}, \ldots, z_{j n}\right)
$$

or

$$
\tau\left(z_{j N}, z_{C}\right)=z_{C}
$$

The fibres $E_{p}$ of the map $\tau: E \rightarrow C$ are isomorphic to $\mathbf{P}^{k-1}$. Let $N_{C / M}$ be the normal bundle of $C$ in $M$ and let $N$ be its restriction to $V \cap C$ :

$$
N=\left.N_{C / M}\right|_{V \cap C} \cong(V \cap C) \times \mathbf{C}^{k} .
$$

There is a natural isomorphism $\psi: E \stackrel{\sim}{\rightarrow} \mathbf{P}\left(N_{C / M}\right)$, given locally as a map $\tilde{V} \cap E \rightarrow \mathbf{P}(N) \cong(V \cap C) \times \mathbf{P}^{k-1}$ by

$$
\psi\left(Z_{N}, Z_{C},[\xi]\right)=\left(Z_{C},[\xi]\right) .
$$

In local coordinates on $U_{j}$ and $\mathbf{P}(N)$, the map $\psi$ is given by

$$
\psi\left(z_{j N}, z_{C}\right)=\left(z_{C},[\zeta(j)]\right)
$$

where $\zeta(j)$ is defined by

$$
\zeta(j)_{i}=z_{j i} \quad 1 \leq i \leq k, \quad i \neq j, \quad \text { and } \quad \zeta(j)_{j}=1
$$

so that $[\zeta(j)]=[\xi]$. The functions $\zeta(j)_{i}$, for $i \neq j$, are nonhomogeneous coordinates for an open set in $\mathbf{P}^{k-1}$ and

$$
z_{j j} \zeta(j)=\left(Z_{1}, \ldots, Z_{k}\right)=Z_{N}
$$

for all $j$.

(3.3) The associated line bundle L. Let $L=[E]$ be the line bundle on $\tilde{M}$ associated with the exceptional divisor $E$. The restriction of $L$ to $E$ is isomorphic to the normal bundle $N_{E / \tilde{M}}$ of $E$ in $\tilde{M}$. The restriction of $L$ to each fibre $E_{p} \cong \mathbf{P}^{k-1}$ of the map $\tau: E \rightarrow C$ is isomorphic to the universal bundle $\mathcal{O}_{\mathbf{P}^{k-1}}(-1)$ on $\mathbf{P}^{k-1}$. If $v$ is a nonzero point in the fibre $N_{C / M, p}$ of $N_{C / M}$ over $p,[v]$ 
is the corresponding point in $\mathbf{P}\left(N_{C / M}\right)_{p}$, and $\psi: E \stackrel{\sim}{\rightarrow} \mathbf{P}\left(N_{C / M}\right)$ is the natural isomorphism, then $\psi^{-1}(p,[v]) \in E_{p}$ and the fibre of $L$ over $\psi^{-1}(p,[v])$ corresponds, via the isomorphism $\left.L\right|_{E_{p}} \cong \mathcal{O}_{E_{p}}(-1)$, to the line in $N_{C / M, p}$ spanned by $v$. We have the following commutative diagram relating $\left.L\right|_{E}$ and $N_{C / M}$ :

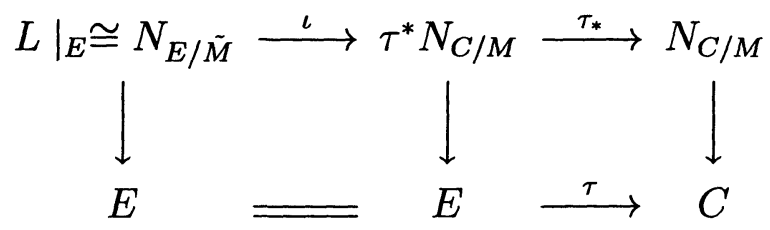

where $\iota$ denotes inclusion.

(3.4) Transition functions and sections of L. We may cover $C$ by coordinate neighbourhoods $V_{\alpha}$ of the form described above and then enlarge this collection of coordinate neighbourhoods to a covering $\mathcal{V}$ of all of $M$. In $\tilde{M}$, the collection of coordinate neighbourhoods $U_{\alpha, j}$ covers $E$ and we enlarge it to a covering $\mathcal{U}$ of $\tilde{M}$. For each $U \in \mathcal{U}$, let $f_{U}$ be a holomorphic defining function for $E$ on $U$. For $U$ and $U^{\prime} \in \mathcal{U}$, let $g_{U U^{\prime}}$ be the nonvanishing holomorphic function on $U \cap U^{\prime}$ given by

$$
g_{U U^{\prime}}=\frac{f_{U}}{f_{U^{\prime}}}
$$

The functions $\left\{g_{U U^{\prime}}\right\}$ are transition functions for a line bundle $L$ on $\tilde{M}$ associated with $E$. If $\left\{F_{U}\right\}$ is another collection of defining functions for $E$, the functions $\left\{G_{U U^{\prime}}=F_{U} / F_{U^{\prime}}\right\}$ may be different from the functions $\left\{g_{U U^{\prime}}\right\}$, but the line bundle determined by the functions $\left\{G_{U U^{\prime}}\right\}$ is isomorphic to $L$.

In particular, $z_{i i}$ is a defining function for $E$ on $U_{i}$, so we may use the functions

$$
g_{i j}=\frac{z_{i i}}{z_{j j}}=\frac{Z_{i}}{Z_{j}}
$$

as transition functions for $L=[\underset{\tilde{M}}{E}]$ on the set $U_{i} \cap U_{j}$.

A meromorphic section $s: \tilde{M} \rightarrow L$ of $L$ may be given by a collection of meromorphic functions $s_{U}$ on $U$ satisfying $s_{U}=g_{U U^{\prime}} s_{U^{\prime}}$ on $U \cap U^{\prime}$. Each meromorphic section $s$ of $L$ defines a divisor $(s)$ which is linearly equivalent to $E$. The functions $f_{U}$ themselves give a global holomorphic section $s$ of $L$ for which $(s)=E$. If $s^{\prime}$ is 
another section of $L$ such that $\left(s^{\prime}\right)=E$ then $s$ and $s^{\prime}$ differ by a meromorphic function with no zeros or poles, i.e. by a constant. In particular, if $(s)=E$ then we may assume that $s_{i}=a z_{i i}$ on $U_{\imath}$ for all $i$ and for some constant $a \in \mathbf{C}$.

(3.5) The Chern form of a metric on $\mathbf{L}$. Let $h$ be a hermitian metric on $L$, let $s: \tilde{M} \rightarrow L$ be a nonzero global holomorphic section of $L$, and let $\|s\|$ be the norm of $s$ under $h$ at any point in $\tilde{M}$. The Chern form of the metric $h$ is the $(1,1)$-form

$$
c_{1}(L, h)=-\frac{\sqrt{-1}}{2 \pi} \partial \bar{\partial} \log \|s\|^{2} .
$$

The following local calculation shows that $c_{1}(L, h)$ is well-defined and is independent of the section $s$ used to calculate it. On any set $U \in \mathcal{U}$ we have $\|s\|^{2}=h_{U}\left|s_{U}\right|^{2}$, for some positive $C^{\infty}$ function $h_{U}$. Then

$$
\begin{aligned}
\partial \bar{\partial} \log \|s\|^{2} & =\partial \bar{\partial} \log \left(h_{U}\left|s_{U}\right|^{2}\right) \\
& =\partial \bar{\partial} \log h_{U}+\partial \bar{\partial} \log s_{U}+\partial \bar{\partial} \log \overline{s_{U}} \\
& =\partial \bar{\partial} \log h_{U}
\end{aligned}
$$

since $\bar{\partial} \log s_{U}=\partial \log \overline{s_{U}}=0$.

Note also that $c_{1}(L, h)$ is hermitian and $d$-closed, by Lemma (1.2.1). The Chern class of a line bundle is always integral (see $[\mathbf{G H}]$ or $[\mathbf{W}])$.

(3.6) Pullbacks of divisors with normal crossings. Let $D$ be a smooth connected analytic hypersurface in $M$, i.e. $D$ is a smooth, reduced, irreducible divisor on $M$. Assume that $D$ has normal crossings with $C$. Then for every $p \in C \cap D$ we may choose local coordinates as in $\S 3.1$ and such that $D$ is given locally by the equation $Z_{\alpha}=0$ for some $\alpha$. In the open set $U_{j}$ in $\tilde{M}$, the pullback divisor $\pi^{*} D$ is given by the equation

$$
\begin{aligned}
z_{j j} z_{j \alpha}=0 & \text { if } 1 \leq \alpha \leq k \text { and } \alpha \neq j \\
z_{j \alpha}=0 & \text { if } \alpha=j \text { or } k+1 \leq \alpha \leq n
\end{aligned}
$$

The strict transform $\tilde{D}$ of $D$ is the closure of $\pi^{-1}(D-C)$ in $\tilde{M}$ and is given locally in the open set $U_{j}$ by the equation $z_{j \alpha}=0$ if 
$j \neq \alpha$. If $j=\alpha$ then $\tilde{D}$ does not intersect $U_{j}$. The divisor $\tilde{D}$ is smooth, reduced, and irreducible and has normal crossings with the exceptional divisor $E$. We may express $\pi^{*} D$ globally in terms of $\tilde{D}$ and $E$ as

$$
\pi^{*} D=\tilde{D}+\delta E
$$

where $\delta=1$ if $C \subset D$ and $\delta=0$ otherwise. If $L_{D}=[D]$ is the line bundle on $M$ associated with $D$, then $\pi^{*} L_{D}=[\tilde{D}] \otimes L^{\delta}$, where $L=[E]$.

Now let $D$ be a divisor on $M$ of the form $D=\sum_{i=1}^{r} a_{i} E_{i}$, where the irreducible components $E_{i}$ of $D$ are smooth and each $a_{i}$ is a positive integer, and such that $D$ and $C$ simultaneously have only normal crossings. Then

$$
\pi^{*} D=\sum_{i=1}^{r} a_{i} \pi^{*} E_{i}=\sum_{i=1}^{r} a_{i} \tilde{E}_{i}+\left(\sum_{i=1}^{r} a_{i} \delta_{i}\right) E
$$

where $\delta_{i}=1$ if $C \subset E_{i}$ and $\delta_{i}=0$ otherwise.

(3.7) Pullbacks of divisors under a sequence of blow-ups. Consider a finite sequence of blow-ups $\left\{\pi_{j}: M_{j} \rightarrow M_{j-1}\right\}$ along smooth centres $C_{j} \subset M_{j-1}$. For $0 \leq k<j$, let $\pi_{j, k}=\pi_{k+1} \circ \pi_{k+2} \circ$ $\ldots \circ \pi_{j}: M_{j} \rightarrow M_{k}$. Let $E_{j}$ be the exceptional divisor of $\pi_{j}$, i.e. $E_{j}=$ $\pi_{j}^{-1}\left(C_{j}\right)$. Let $D_{j}$ be the exceptional divisor of the composite $\pi_{j, 0}$ of the first $j$ blow-ups, given inductively by the equations $D_{1}=E_{1}$ and $D_{j}=\pi_{j}^{*} D_{j-1}+E_{j}$ for $j \geq 2$. Assume that the centres $C_{j}$ have been chosen so that $C_{j}$ and $D_{j-1}$ simultaneously have only normal crossings. For $1 \leq j<m$ let $E_{m, j}$ be the pullback of $E_{j}$ to $M_{m}$, i.e. $E_{m, j}$ is the total transform $E_{m, j}=\pi_{m, j}^{*} E_{j}$. Let $E_{m, m}=E_{m}$. We may write $D_{m}$ as the sum

$$
D_{m}=\sum_{j=1}^{m} E_{m, j}
$$

For $1 \leq k<m$, let $\tilde{E}_{m, k}$ be the smooth, reduced, irreducible divisor in $M_{m}$ obtained by taking repeated strict transforms of $E_{k}$ under the blow-ups $\pi_{k+1}, \ldots, \pi_{m}$. Let $\tilde{E}_{m, m}=E_{m}$. The divisors $\left\{\tilde{E}_{m, k}\right\}_{k=1}^{m}$ have only normal crossings. We may express the total transforms $\left\{E_{m, j}\right\}$ in terms of the strict transforms $\left\{\tilde{E}_{m, k}\right\}$ as 


$$
E_{m, j}=\sum_{k=1}^{m} a_{j k} \tilde{E}_{m, k}
$$

for some nonnegative integers $a_{j k}$. Let $A$ be the $m \times m$ matrix $\left(a_{j k}\right)$. We will sometimes write this transition matrix as $A=T_{m}(E, \tilde{E})$. Repeated use of formula (3.6.2) shows that $A$ is an upper triangular matrix of the form

$$
A=T_{m}(E, \tilde{E})=\left(\begin{array}{cccccc}
1 & a_{12} & a_{13} & \ldots & a_{1, m-1} & a_{1 m} \\
0 & 1 & a_{23} & \ldots & a_{2, m-1} & a_{2 m} \\
0 & 0 & 1 & \ldots & a_{3, m-1} & a_{3 m} \\
\vdots & \vdots & \vdots & \ddots & \vdots & \vdots \\
0 & 0 & 0 & \ldots & 1 & a_{m-1, m} \\
0 & 0 & 0 & \ldots & 0 & 1
\end{array}\right) .
$$

Let $\hat{A}=T_{m-1}(E, \tilde{E})$ be the matrix which describes the collection of total transforms $\left\{E_{m-1, j}\right\}_{j=1}^{m-1}$ in terms of the strict transforms $\left\{\tilde{E}_{m-1, j}\right\}_{j=1}^{m-1}$ on $M_{m-1}$. For $1 \leq k \leq j-1$ define

$$
\delta_{k j}= \begin{cases}1 & \text { if } C_{j} \subset \tilde{E}_{j-1, k} \\ 0 & \text { if } C_{j} \not \subset \tilde{E}_{j-1, k} .\end{cases}
$$

Using (3.6.2) we obtain the following relationships between the entries of $A$ and $\hat{A}$ :

LEMMA 3.7.1.

i. $\quad a_{j k}=\hat{a}_{j k}$

if $1 \leq j, k \leq m-1$.

ii.

$$
\begin{aligned}
a_{j m} & =\sum_{k=1}^{m-1} \hat{a}_{j k} \delta_{k m} \quad \text { if } 1 \leq j \leq m-1 \\
& = \begin{cases}\delta_{j m}+\sum_{k=j+1}^{m-1} a_{j k} \delta_{k m} & \text { if } 1 \leq j \leq m-2 \\
\delta_{m-1, m} & \text { if } j=m-1 .\end{cases}
\end{aligned}
$$

Property (i) means that we may omit the superscripts ^on the $a$ 's without any ambiguity: $a_{j k}$ is the multiplicity of $\tilde{E}_{m, k}$ in $E_{m, j}$ and is the same for all $m \geq k$. Property (ii) implies that if $C_{m} \subset E_{m-1, j}$ then $a_{j m}>0$. 
Similarly, we define the total transforms $D_{m, i}$ of the divisors $D_{i}$ by the equations $D_{m, i}=\pi_{m, i}^{*} D_{i}$ for $1 \leq i \leq m-1$ and $D_{m, m}=$ $D_{m}=D_{m, m-1}+E_{m}$. Then

$$
D_{m, i}=\sum_{j=1}^{i} E_{m, j}
$$

and the matrix $T_{m}(D, E)$ for the total transforms $\left\{D_{m, i}\right\}$ in terms of the total transforms $\left\{E_{m, j}\right\}$ is the $m \times m$ lower triangular matrix

$$
T_{m}(D, E)=\left(\begin{array}{cccc}
1 & 0 & \ldots & 0 \\
1 & 1 & \ldots & 0 \\
\vdots & \vdots & \ddots & \ddots \\
1 & 1 & \ldots & 1
\end{array}\right)
$$

The irreducible components of $D_{m}=D_{m, m}$ are the strict transforms $\left\{\tilde{E}_{m, j}\right\}_{j=1}^{m}$. The irreducible components of $D_{m, i}$ for $1 \leq i \leq m-1$ are also contained in the set $\left\{\tilde{E}_{m, j}\right\}$. We may express the divisors $\left\{D_{m, i}\right\}$ in terms of the strict transforms $\left\{\tilde{E}_{m, j}\right\}$ as

$$
D_{m, i}=\sum_{j=1}^{m} t_{i j} \tilde{E}_{m, j}
$$

for some nonnegative integers $t_{i j}$. The matrix $T=\left(t_{i j}\right)=T_{m}(D, \tilde{E})$ is given by

$$
\begin{aligned}
T & =T_{m}(D, E) T_{m}(E, \tilde{E}) \\
& =\left(\begin{array}{cccccc}
1 & a_{12} & a_{13} & \ldots & a_{1, m-1} & a_{1 m} \\
1 & a_{12}+1 & a_{13}+a_{23} & \ldots & a_{1, m-1}+a_{2, m-1} & a_{1 m}+a_{2 m} \\
\vdots & \vdots & \vdots & \ddots & \vdots & \vdots \\
1 & a_{12}+1 & a_{13}+a_{23}+1 \ldots & \sum_{k=1}^{m-2} a_{k, m-1}+1 & \sum_{k=1}^{m-1} a_{k, m} \\
1 & a_{12}+1 & a_{13}+a_{23}+1 \ldots & \sum_{k=1}^{m-2} a_{k, m-1}+1 & \sum_{k=1}^{m-1} a_{k, m}+1
\end{array}\right) .
\end{aligned}
$$

Let $\hat{T}=T_{m-1}(D, \tilde{E})$ be the matrix for the divisors $\left\{D_{m-1, i}\right\}$ in terms of the strict transforms $\left\{\tilde{E}_{m-1, j}\right\}$. 
LEMMA 3.7.3. The matrices $T=\left(t_{i j}\right)=T_{m}(D, \tilde{E})$ and $\hat{T}=$ $\left(\hat{t}_{i j}\right)=T_{m-1}(D, \tilde{E})$ are related by the following equations:

i. $t_{i j}=\hat{t}_{i j}$ for $1 \leq i, j \leq m-1$,

ii. $t_{i m}=\sum_{k=1}^{m-1} t_{i k} \delta_{k m}$ for $1 \leq i \leq m-1$,

iii. $t_{m j}=t_{m-1, j}=\hat{t}_{m-1, j}$ for $1 \leq j \leq m-1$, and

iv. $t_{m m}=t_{m-1, m}+1=\sum_{k=1}^{m-1} t_{m-1, k} \delta_{k m}+1=\sum_{k=1}^{m-1} t_{m k} \delta_{k m}+1$.

Proof. Apply equation (3.6.2) to the pullbacks $\pi_{m}^{*} D_{m-1, i}=D_{m, i}$ for $1 \leq i \leq m-1$ to obtain equations (i) and (ii). The exceptional divisor $D_{m, m}$ is just

$$
D_{m}=\pi_{m}^{*} D_{m-1, m-1}+E_{m}=D_{m, m-1}+\tilde{E}_{m, m} .
$$

Applying (3.6.2) again gives equations (iii) and (iv).

Using equation (3.7.2) and Lemma (3.7.3) we obtain the following description of $T=T_{m}(D, \tilde{E})$ for $m \geq 2$.

Proposition 3.7.4. The matrix $T=\left(t_{i j}\right)$ given by the equations

$$
D_{m, i}=\sum_{j=1}^{m} t_{i j} \tilde{E}_{m, j}
$$

has the following properties:

i. $t_{i j}$ is a nonnegative integer and $t_{i j}>0$ if $i \geq j$,

ii. $t_{i j} \geq t_{k j}$ if $i \geq k$,

iii. $t_{m j}=t_{m-1, j}=\ldots=t_{j+1, j}=t_{j j}$,

iv. $\operatorname{det} T=1$,

v. $t_{j-1, j}>0$ if and only if $C_{j} \subset D_{j-1}$, and

vi. the entries of $T$ are all positive if and only if $C_{j} \subset D_{j-1}$ for $2 \leq j \leq m$.

Proof. Properties (i) - (iii) are immediate consequences of equation (3.7.2). To prove (iv), note that $\operatorname{det} T_{m}(D, E)=\operatorname{det} T_{m}(E, \tilde{E})=$ 1. For (v), use Lemma (3.7.3ii) to write $t_{j-1, j}$ as

$$
t_{j-1, j}=\sum_{k=1}^{j-1} t_{j-1, k} \delta_{k j}
$$


where the coefficients $t_{j-1, k}$ are positive for $1 \leq k \leq j-1$ by property (i). Recall that $\delta_{k j}>0$ if and only if $C_{j} \subset \tilde{\tilde{E}}_{j-1, k}$. Then $t_{j-1, j}>0$ if and only if $C_{j} \subset \tilde{E}_{j-1, k}$ for some $k, 1 \leq k \leq j-1$. The divisors $\left\{\tilde{E}_{j-1, k}\right\}_{k=1}^{j-1}$ are the irreducible components of $D_{j-1}$, so $t_{j-1, j}>0$ if and only if $C_{j} \subset D_{j-1}$.

It follows from (v) that if $C_{j} \not \subset D_{j-1}$ for some $j$ then not all entries of $T$ are positive. If $m=2$ we have

$$
T_{2}=\left(\begin{array}{ll}
t_{11} & t_{12} \\
t_{21} & t_{22}
\end{array}\right)=\left(\begin{array}{cc}
1 & a_{12} \\
1 & a_{12}+1
\end{array}\right)
$$

where $t_{12}$ is positive if and only if $C_{2} \subset D_{1}$, by (v). Suppose that for some $j \geq 3$ the matrix $T_{j-1}$ has positive entries and $C_{i} \subset D_{i-1}$ for $2 \leq i \leq j$. The matrix $T_{j}$ is of the form

$$
T_{j}=\left(\begin{array}{ccc|c} 
& & & t_{1 j} \\
& T_{j-1} & \vdots \\
& & t_{j-1, j} \\
\hline t_{j 1} & \cdots & t_{j, j-1} & t_{j j}
\end{array}\right)
$$

where $\left(t_{j 1}, t_{j 2}, \ldots, t_{j, j-1}\right)=\left(t_{j-1,1}, t_{j-1,2}, \ldots, t_{j-1, j-1}\right)$, by property (iii), and $t_{i j} \geq t_{k j}$ for $i \geq k$ by property (ii). From these properties and our inductive assumption, it is sufficient to show that $t_{1 j}>0$. By Lemma (3.7.3ii), $t_{1 j}=\sum_{k=1}^{j-1} t_{1 k} \delta_{k j}$. The coefficients $t_{1 k}$ are positive for $1 \leq k \leq j-1$ by our inductive assumption. If $C_{j} \subset D_{j-1}$ then $C_{j} \subset \tilde{E}_{j-1, k}$ and $\delta_{k j}=1$ for some $k, 1 \leq k \leq j-1$. Consequently $t_{1 j}>0$.

When working in local coordinates near a point $q$ in $M_{m}$ we will consider only the submatrix $\Lambda$ of $T_{m}$ corresponding to those divisors $\tilde{E}_{m, \alpha}$ which pass through $q$.

(3.8) Isolated singularities. If $X$ has only isolated singular points, then we may resolve each singular point in turn and write a separate matrix for the exceptional divisor of each, since each blow-up map $\pi_{i}$ will be a biholomorphism on all connected components of $D_{i}$ except the one containing $E_{i}$. The exceptional divisors for distinct points will be mutually disjoint. For our purposes it is enough to describe the matrix corresponding to a single singular point of $X$. 
Proposition 3.8.1. Let $X$ be a singular compact analytic subspace of $M_{0}$ with isolated singularities. Let $\left\{\pi_{m}: M_{m} \rightarrow M_{m-1}\right\}$ be a sequence of blow-ups of the type described in $\S 1.1$ which resolves one of the singular points of $X$. Then, for each $m$, all entries of the matrix $T=T_{m}(D, \tilde{E})$ are positive, i.e. the multiplicity of the strict transform $\tilde{E}_{m, i}$ of $E_{i}$ in the total transform $D_{m, j}$ of $D_{j}$ is positive for all $i, j$.

Proof. Suppose that the blow-ups have the properties described in $\S 1.1$. Then the image of $D_{j}$ in $M_{0}$ always lies in $X_{\text {sing }}$, so $C_{1} \subset X_{\text {sing }}$ and $C_{j} \subset D_{j-1}$ for $j \geq 2$. Apply Proposition (3.7.4).

(3.9) Line bundles for repeated pullbacks. We list here, for further reference, the relationships among certain divisors, line bundles, sections, and metrics.

Let $h_{j}$ be a hermitian metric for the line bundle $L_{j}=\left[E_{j}\right]$ on $M_{j}$ and let $s_{j}: M_{j} \rightarrow L_{j}$ be a section of $L_{j}$ such that $\left(s_{j}\right)=E_{j}$. Such a section always exists $(\S 3.4)$. For $m>j$ let $\pi_{m, j}$ be the composite map

$$
\pi_{m, j}=\pi_{j+1} \circ \pi_{j+2} \circ \ldots \circ \pi_{m}: M_{m} \rightarrow M_{j} .
$$

Recall that the total exceptional divisor of $\pi_{j, 0}$ is $D_{j}=\sum_{i=1}^{j} E_{j, i}$. Let $\hat{L}_{j}=\left[D_{j}\right]$. The hermitian metrics $h_{j}$ and the sections $s_{j}$ induce hermitian metrics $\hat{h}_{j}$ and sections $\hat{s}_{j}$ of the line bundles $\hat{L}_{j}$. We use the notation

$$
\begin{aligned}
& L_{m, j}=\pi_{m, j}^{*} L_{j}, E_{m, j}=\pi_{m, j}^{*} E_{j}, h_{m, j}=\pi_{m, j}^{*} h_{j}, \\
& \hat{L}_{m, j}=\pi_{m, j}^{*} \hat{L}_{j}, D_{m, j}=\pi_{m, j}^{*} D_{j}, \hat{h}_{m, j}=\pi_{m, j}^{*} \hat{h}_{j}, \text { and } \quad \hat{s}_{m, j}^{*}=\pi_{m, j}^{*} s_{j} \hat{s}_{j} .
\end{aligned}
$$

Then

$$
\begin{aligned}
\hat{L}_{j} & =\bigotimes_{i=1}^{j} L_{j, i}, \quad \hat{h}_{j}=\prod_{i=1}^{j} h_{j, i}, \quad \hat{s}_{j}=\prod_{i=1}^{j} s_{j, i}, \quad\left(\hat{s}_{j}\right)=D_{j}, \\
L_{m, j} & =\left[E_{m, j}\right], \quad\left(s_{m, j}\right)=E_{m, j}, \quad \hat{L}_{m, j}=\left[D_{m, j}\right], \text { and } \quad\left(\hat{s}_{m, j}\right)=D_{m, j} .
\end{aligned}
$$

For consistency we sometimes write $L_{m, m}=L_{m}, E_{m, m}=E_{m}$, $D_{m, m}=D_{m}$, and so on. 


\section{$\S 4$. An Incomplete Metric on $M-X_{\text {sing }}$ which determines} an Embedded Resolution of $X$ by Blow-ups. Let $M$ be a compact complex manifold of dimension $n$, let $C$ be a submanifold of codimension $k>1$, and let $\pi: \tilde{M} \rightarrow M$ be the blow-up of $M$ along $C$. Let $E=\pi^{-1}(C)$ be the exceptional divisor of the blowup and let $L=[E]$ be its associated line bundle on $\tilde{M}$. We begin with a hermitian metric $h_{M}$ on $M$, or equivalently a $C^{\infty}$ positive hermitian $(1,1)$-form $\omega$. We will show that if $c_{1}(L, h)$ is the Chern form of $L$ with respect to a suitable hermitian metric $h$ on $L$ and $l$ is a sufficiently large integer, then the $(1,1)$-form

$$
\tilde{\omega}=l \pi^{*} \omega-c_{1}(L, h)
$$

is positive and determines a hermitian metric $h_{\tilde{M}}$ on $\tilde{M}$. If $h_{M}$ is Kähler then so is $h_{\tilde{M}}$. If $M$ is algebraic and $h_{M}$ is Hodge, then $h_{\tilde{M}}$ is also Hodge.

Applying this construction inductively to a sequence of blow-ups $\pi_{j}: M_{j} \rightarrow M_{j-1}$, we obtain $C^{\infty}$ positive hermitian $(1,1)$-forms

$$
\omega_{j}=l_{j} \pi_{j}^{*} \omega_{j-1}-c_{1}\left(L_{j}, h_{j}\right)
$$

which determine hermitian metrics $h_{M_{j}}$ on $M_{j}$. Suppose that $X$ is an analytic subspace of $M_{0}$ and the blow-ups $\pi_{j}$ are of the type described in $\S 1.1$. Then the restrictions of the metrics $h_{M_{j}}$ to the strict transforms $X_{j}$ of $X$ induce hermitian metrics on $X-X_{\text {sing }}$ which are Kähler if $h_{M_{0}}$ is Kähler but are incomplete unless $X$ is nonsingular. Moreover the completion of $M_{j}-D_{j}$ in the metric $h_{M_{j}}$ is $M_{j}$ and the completion of $X-X_{\text {sing }}$ is $X_{j}$.

(4.1) A metric for $L$. Let $\tau: E \rightarrow C$ be the restriction of the map $\pi: \tilde{M} \rightarrow M$ to E. For any subset $W \subset \tilde{M}$, let $L_{W}$ be the restriction of the line bundle $L$ to $W$.

Proposition 4.1.1. There exists a hermitian metric $h$ on $L$ whose Chern form $c_{1}(L, h)$ is negative along the fibres of the map $\tau: E \rightarrow C$, i.e. the restriction of $c_{1}(L, h)$ to the tangent bundle $T\left(E_{p}\right)$ of each fibre $E_{p}$ of $\tau$ is a negative definite $(1,1)$-form.

Proof. Let $N=N_{C / M}$ be the normal bundle of $C$ in $M$. Any hermitian metric on $N$ induces a hermitian metric on $L_{E}$ by first pulling back to $\tau^{*} N$ and then restricting (see diagram (3.3.1)). A 
metric on $L_{E}$ may be extended in a $C^{\infty}$ way to a metric on $L$ over all of $\tilde{M}$, for example by using the tubular neighbourhood construction of Proposition (A.3). We will show that any hermitian metric constructed in this way has the required property.

Let $p$ be any point in $C$. Choose local coordinates $\left(Z_{1}, \ldots, Z_{n}\right)$ in a coordinate neighbourhood $V$ in $M$ centered at $p$ and local coordinates $\left(z_{j 1}, \ldots, z_{j n}\right)$ on sets $U_{j}$ in $\tilde{M}$, as described in $\S 3.1$. We will denote points in $N$ over $V \cap C$ by $\left(Z_{C}, \xi\right)$ where $Z_{C}=\left(Z_{k+1}, \ldots, Z_{n}\right) \in$ $C$ and

$$
\xi=\sum_{i=1}^{k} \xi_{i} \frac{\partial}{\partial Z_{i}}
$$

Choose any hermitian metric \|\| on $N$. In local coordinates,

$$
\left\|\left(Z_{C}, \xi\right)\right\|^{2}=\sum_{\mu, \nu=1}^{k} h_{\mu \nu}\left(Z_{C}\right) \xi_{\mu} \overline{\xi_{\nu}}
$$

for some $C^{\infty}$ functions $h_{\mu \nu}$ such that the matrix $\left(h_{\mu \nu}\right)$ is positive definite hermitian. We may make a linear change of variables in $Z_{1}, \ldots, Z_{k}$ (and hence in $\left.\xi_{1}, \ldots, \xi_{k}\right)$ so that at $p=(0,0, \ldots, 0)$, the matrix $\left(h_{\mu \nu}(0)\right)$ is the $k \times k$ identity and

$$
\|(0, \xi)\|^{2}=\sum_{\mu=1}^{k}\left|\xi_{\mu}\right|^{2}
$$

Next we describe the induced metric on $L_{E}$. We denote points in $L$ over $U_{j}$ by $\left(\left(z_{j N}, z_{C}\right), t_{j}\right)$ and note that on $U_{i} \cap U_{j}$ the fibre coordinate transforms by the rule

$$
t_{i}=g_{i j} t_{j}
$$

where $g_{i j}=z_{i i} / z_{j j}$ is the transition function for $L$ on $U_{i} \cap U_{j}$. The natural map $L_{E} \rightarrow N$ is given locally by

$$
\left(\left(z_{j N}, z_{C}\right), t_{j}\right) \rightarrow\left(z_{C}, t_{j} \zeta(j)\right)
$$

where $\zeta(j)$ is as defined in $\S 3.2$, i.e. $\zeta(j)_{i}=z_{j i}$ for $1 \leq i \leq k, i \neq j$, and $\zeta(j)_{j}=1$. The hermitian metric on $L_{E}$ induced from $N$ is given locally by

$$
\left\|\left(\left(z_{j N}, z_{C}\right), t_{j}\right)\right\|^{2}=\left|t_{j}\right|^{2} \sum_{\mu, \nu=1}^{k} h_{\mu \nu}\left(z_{C}\right) \zeta(j)_{\mu} \overline{\zeta(j)_{\nu}}
$$


On the fibre $E_{p}$ of the map $\tau: E \rightarrow C$, we have $z_{C}=0$ and

$$
\left\|\left(\left(z_{j N}, 0\right), t_{j}\right)\right\|^{2}=\left|t_{j}\right|^{2} \sum_{\mu=1}^{k}\left|\zeta(j)_{\mu}\right|^{2} .
$$

Let $h$ be any $C^{\infty}$ extension of this metric to a metric on $L$ over all of $\tilde{M}$, for example by using the tubular neighbourhood construction of Proposition (A.3). To finish the proof of the proposition, we need only look at the description of $h$ on $E$. The restriction of $c_{1}(L, h)$ to the tangent bundle $T E$ of $E$ may be calculated using the formulas of $\S 3.5$ :

$$
\left.c_{1}(L, h)\right|_{T\left(E \cap U_{j}\right)}=-\frac{\sqrt{-1}}{2 \pi} \partial \bar{\partial} \log \left(\sum_{\mu, \nu=1}^{k} h_{\mu \nu}\left(z_{C}\right) \zeta(j)_{\mu} \overline{\zeta(j)_{\nu}}\right) .
$$

The restriction of $c_{1}(L, h)$ to the tangent bundle of the fibre $E_{p}$ is

$$
\left.c_{1}(L, h)\right|_{T\left(E_{p}\right)}=-\frac{\sqrt{-1}}{2 \pi} \partial \bar{\partial} \log \sum_{\mu=1}^{k}\left|\zeta(j)_{\mu}\right|^{2}
$$

which is a negative $(1,1)$-form, the negative of the $(1,1)$-form associated to the Fubini-Study metric on $\mathbf{P}^{k-1}$. Since $p$ was any point of $C$, the Chern form $c_{1}(L, h)$ is negative along every fibre of the $\operatorname{map} \tau: E \rightarrow C$.

\section{(4.2) A finite metric for $\tilde{M}$.}

Proposition 4.2.1. If $\omega$ is a positive $(1,1)$-form on $M$, and $h$ is a hermitian metric on $L$ whose Chern form is negative on the fibres of the map $\tau: E \rightarrow C$, then the $(1,1)$-form

$$
\tilde{\omega}=l \pi^{*} \omega-c_{1}(L, h)
$$

is positive on $\tilde{M}$ for all sufficiently large integers $l$.

Proof. Let $Y$ be the subspace of $\left.T \tilde{M}\right|_{E}$ consisting of all vectors which are tangent to fibres of the map $\tau: E \rightarrow C$, i.e.

$$
Y=\bigcup_{c \in C} T\left(\pi^{-1}(c)\right) .
$$


If $\omega$ is a positive $(1,1)$-form on $M$, then $\pi^{*} \omega$ is positive semi-definite on $\tilde{M}$. If $v \in T \tilde{M}$ is a nonzero tangent vector then $\pi^{*} \omega(v, v)=0$ if and only if $v \in Y$. The Chern form $c_{1}(L, h)$ is negative on $Y$ by construction. These properties can be expressed in terms of functions on the projectivized tangent bundle $P=\mathbf{P} T \tilde{M}$.

Let $\mathrm{h}^{\prime}$ be any hermitian metric on $T \tilde{M}$ and for $v$ in $T \tilde{M}$ let $\|v\|$ be the norm of $v$ under $\mathrm{h}^{\prime}$. For $v \neq 0$, let $[v]$ be the image of $v$ in $P$. The $(1,1)$-forms $\pi^{*} \omega$ and $-c_{1}(L, h)$ determine well-defined $C^{\infty}$ functions on $P$ given by

$$
f([v])=\frac{\pi^{*} \omega(v, v)}{\|v\|^{2}} \quad \text { and } \quad g([v])=\frac{-c_{1}(L, h)(v, v)}{\|v\|^{2}}
$$

with the properties $f>0$ on $P-\mathbf{P} Y, f=0$ on $\mathbf{P} Y$, and $g>0$ on $\mathbf{P} Y$. Since $\mathbf{P} Y$ is closed, $g>0$ on some neighbourhood $U$ of $\mathbf{P} Y$. Then $l f+g>0$ on $U$ for all $l>0$. Since $P$ is compact, we may choose $l>0$ such that $l f+g>0$ on all of $P$, and consequently $l \pi^{*} \omega-c_{1}(L, h)>0$ on $\tilde{M}$ for all sufficiently large $l$.

Proposition (4.1.1) tells us that there is always a metric $h$ on $L$ with the properties required in Proposition (4.2.1) and Theorem (4.2.2).

THEOREM 4.2.2. Let $\omega$ be the fundamental form of a hermitian metric on $M$ and let $h$ be a hermitian metric on $L$ whose Chern form is negative on the fibres of the map $\tau: E \rightarrow C$. Then

$$
\tilde{\omega}=l \pi^{*} \omega-c_{1}(L, h)
$$

is the fundamental form of a hermitian metric on $\tilde{M}$ for all sufficiently large integers $l$. If $\omega$ is Kähler then so is $\tilde{\omega}$ and if $\omega$ is Hodge then so is $\tilde{\omega}$.

Proof. A $C^{\infty}(1,1)$-form is the fundamental form of a hermitian metric if it is positive and hermitian. The positivity of $\tilde{\omega}$ was proved above (Proposition (4.2.1)). The Chern form of a line bundle is always hermitian (§3.5), so $\tilde{\omega}$ is also hermitian.

Recall that a positive hermitian $C^{\infty}(1,1)$-form $\omega$ determines a Kähler metric if $d \omega=0$. If $\omega$ is also integral, then the metric is Hodge. The identity $d\left(\pi^{*} \omega\right)=\pi^{*} d \omega$ shows that $\pi^{*} \omega$ is $d$-closed if $\omega$ is $d$-closed and $\pi^{*} \omega$ is integral if $\omega$ is integral. Finally, the Chern form of a line bundle is always $d$-closed and integral ( $§ 3.5)$. 
(4.3) Finite metrics for successive blow-ups. We apply Theorem (4.2.2) to a sequence of blow-ups to obtain the following.

THEOREM 4.3.1. Let $\left\{\pi_{j}: M_{j} \rightarrow M_{j-1}\right\}$ be a finite sequence of blow-ups of a compact complex manifold $M_{0}$ along smooth centres $C_{j} \subset M_{j-1}$. Let $E_{j}=\pi_{j}^{-1}\left(C_{j}\right)$ be the exceptional divisor of $\pi_{j}$ and let $L_{j}=\left[E_{j}\right]$ be the associated line bundle. Let $\omega_{0}$ be the fundamental form of a hermitian metric on $M_{0}$. There exist hermitian metrics $h_{j}$ on $L_{j}$ and positive integers $l_{j}$ such that the $(1,1)$-forms defined inductively by the equation

$$
\omega_{j}=l_{j} \pi_{j}^{*} \omega_{j-1}-c_{1}\left(L_{j}, h_{j}\right)
$$

are all positive and determine hermitian metrics on the manifolds $M_{j}$. Moreover if $\omega_{0}$ is Kähler then so are all the forms $\omega_{j}$. If $M_{0}$ is algebraic and $\omega_{0}$ is Hodge then the forms $\omega_{j}$ are also Hodge.

REMARK. Since a sum of Chern forms equals the Chern form of a product of line bundles, we may write $\omega_{j}$ as

$$
\omega_{j}=l \pi_{j, 0}^{*} \omega_{0}-c_{1}(\mathcal{L}, H)
$$

where $\pi_{j, 0}: M_{j} \rightarrow M_{0}$ is the composite of the first $j$ blow-ups, $\mathcal{L}$ is some line bundle on $M_{j}$, and $H$ is an appropriate metric on $\mathcal{L}$. Furthermore, $\mathcal{L}$ is of the form $\mathcal{L}=[\mathcal{D}]$ for some effective divisor $\mathcal{D}$ with the same support as $D_{j}$.

We may also obtain Kähler metrics inductively using the divisors $D_{j}$ as follows. Corollary (4.3.2) will be used in $\S 8.6$ in the proof of our first main theorem.

Corollary 4.3.2. Let $D_{j}$ be the exceptional divisor of the composite $\pi_{1} \circ \pi_{2} \circ \ldots \circ \pi_{j}$ of the first $j$ blow-ups, i.e. $D_{1}=E_{1}$ and $D_{j}=\pi_{j}^{*} D_{j-1}+E_{j}$ for $j \geq 2$. There exist hermitian metrics $\hat{h}_{j}$ on the line bundles $\left[D_{j}\right]$ and positive integers $l_{j}$ such that the $(1,1)$ forms defined inductively by $\hat{\omega}_{0}=\omega_{0}$ and

$$
\hat{\omega}_{j}=l_{j} \pi_{j}^{*} \hat{\omega}_{j-1}-c_{1}\left(\left[D_{j}\right], \hat{h}_{j}\right) \quad \text { for } j \geq 1
$$

are all positive and determine hermitian metrics on the manifolds $M_{j}$. Moreover if $\omega_{0}$ is Kähler (resp. Hodge) then $\hat{\omega}_{j}$ is Kähler (resp. Hodge) for all $j$.

Proof. We need only prove positivity. Let $\hat{L}_{j}=\left[D_{j}\right]$, let $h_{j}$ be a hermitian metric on $L_{j}=\left[E_{j}\right]$ whose Chern form is negative on the 
fibres of the map $E_{j} \rightarrow C_{j}$, and define metrics $\hat{h}_{j}$ on $\hat{L}_{j}$ inductively by the equations $\hat{h}_{1}=h_{1}$ and $\hat{h}_{j}=\left(\pi_{j}^{*} \hat{h}_{j-1}\right) h_{j}$. For $j=1$ we have $\left[D_{1}\right]=\left[E_{1}\right]=L_{1}$ and positivity follows from Theorem (4.3.1). Assume that $\hat{\omega}_{j-1}>0$ on $M_{j-1}$ for some $j \geq 2$. Using the inductive descriptions of $D_{j}$ and $\hat{h}_{j}$ we obtain

$$
\begin{aligned}
c_{1}\left(\left[D_{j}\right], \hat{h}_{j}\right) & =c_{1}\left(\hat{L}_{j}, \hat{h}_{j}\right) \\
& =c_{1}\left(\left(\pi_{j}^{*} \hat{L}_{j-1}\right) \otimes L_{j},\left(\pi_{j}^{*} \hat{h}_{j-1}\right) h_{j}\right) \\
& =\pi_{j}^{*}\left(c_{1}\left(\hat{L}_{j-1}, \hat{h}_{j-1}\right)\right)+c_{1}\left(L_{j}, h_{j}\right) .
\end{aligned}
$$

Now $l^{\prime} \hat{\omega}_{j-1}-c_{1}\left(\hat{L}_{j-1}, \hat{h}_{j-1}\right)>0$ for $l^{\prime} \gg 0$ because $\hat{\omega}_{j-1}>0$ and $c_{1}\left(\hat{L}_{j-1}, \hat{h}_{j-1}\right)$ is bounded on $M_{j-1}$. Therefore

$$
\pi_{j}^{*}\left(l^{\prime} \hat{\omega}_{j-1}-c_{1}\left(\hat{L}_{j-1}, \hat{h}_{j-1}\right)\right) \geq 0
$$

on $M_{j}$. By Proposition (4.2.1), our inductive assumption that $\hat{\omega}_{j-1}>$ 0 , and our choice of the metric $h_{j}$, we have

$$
l^{\prime \prime} \pi_{j}^{*} \hat{\omega}_{j-1}-c_{1}\left(L_{j}, h_{j}\right)>0
$$

for $l^{\prime \prime} \gg 0$. Let $l_{j}=l^{\prime}+l^{\prime \prime}$.

(4.4) More finite metrics. In this section we construct a family of Kähler forms $\psi_{1}, \ldots, \psi_{m}$ on $M_{m}$ which will be used in $\S 9$ to construct Kähler homogeneous Saper metrics.

Proposition 4.4.1. There exist divisors $\mathcal{D}_{1}, \ldots, \mathcal{D}_{m}$ on $M_{m}$ of the form

$$
\mathcal{D}_{j}=\sum_{k=1}^{m} b_{j k} \tilde{E}_{m, k},
$$

and metrics $H_{j}$ on the line bundles $\left[\mathcal{D}_{j}\right]$, such that the transition matrix $T(\mathcal{D}, \tilde{E})=\left(b_{j k}\right)$ is a nonsingular matrix of positive integers and such that the forms

$$
\psi_{j}=r \pi_{m, 0}^{*} \omega_{0}-c_{1}\left(\left[\mathcal{D}_{j}\right], H_{j}\right)
$$

are Kähler forms on $M_{m}$ for all sufficiently large integers $r$.

Proof. First we write the Kähler forms $\omega_{j}$ of $\S 4.3$ in terms of the pullback to $M_{j}$ of $\omega_{0}$ and the Chern forms of certain line bundles on $M_{j}$. Recall that in $\S 3.9$ we defined

$$
\pi_{j, i}=\pi_{i+1} \circ \pi_{i+2} \circ \ldots \circ \pi_{j}: M_{j} \rightarrow M_{i}
$$


and the corresponding pullbacks

$$
L_{j, i}=\pi_{j, i}^{*} L_{i}=\left[E_{j, i}\right]=\left[\pi_{j, i}^{*} E_{i}\right]
$$

for $j>i$. We set $L_{j, j}=L_{j}$ and $E_{j, j}=E_{j}$. The induced metric on $L_{j, i}$ is $h_{j, i}=\pi_{j, i}^{*} h_{i}$ but for simplicity we will omit the metric and write $c_{1}\left(L_{j, i}\right)$ or $c_{1}\left(\left[E_{j, i}\right]\right)$ for the Chern form $c_{1}\left(L_{j, i}, h_{j, i}\right)$. Using the integers $l_{i}$ of Theorem (4.3.1) and letting $r_{j}=l_{1} l_{2} \ldots l_{,}, r_{, i}=$ $l_{i+1} l_{i+2} \ldots l_{j}$ for $1 \leq i \leq j-1$, and $r_{j j}=1$ we may write $u_{,}$, as

$$
\begin{aligned}
\omega_{j} & =r_{j} \pi_{j, 0}^{*} \omega_{0}-\sum_{i=1}^{j} r_{j i} c_{1}\left(L_{j, i}\right) \\
& =r_{j} \pi_{j, 0}^{*} \omega_{0}-c_{1}\left(\left[\sum_{i=1}^{j} r_{j i} E_{j, i}\right]\right) .
\end{aligned}
$$

There is a similar formula for $\hat{\omega}_{j}$.

For $m>j$ the pullback of $\omega_{j}$ to $M_{m}$ is a positive semi-definite form given by

$$
\begin{aligned}
\pi_{m, j}^{*} \omega_{j} & =r_{j} \pi_{m, 0}^{*} \omega_{0}-\sum_{k=1}^{j} r_{j k} c_{1}\left(L_{m, k}\right) \\
& =r_{j} \pi_{m, 0}^{*} \omega_{0}-c_{1}\left(\left[\sum_{k=1}^{j} r_{j k} E_{m, k}\right]\right) .
\end{aligned}
$$

The form $\omega_{m}$ is positive and has a similar formula. Let $D_{j}^{\prime}=$ $\sum_{k=1}^{j} r_{j k} E_{m, k}$ for $1 \leq j \leq m$ and let $c_{1}\left(\left[D_{j}^{\prime}\right]\right)$ be the Chern form with respect to the induced metric on the line bundle $\left[D_{j}^{\prime}\right]$. Then

$$
\pi_{m, j}^{*} \omega_{j}=r_{j} \pi_{m, 0}^{*} \omega_{0}-c_{1}\left(\left[D_{j}^{\prime}\right]\right)
$$

for $m>j$ and

$$
\omega_{m}=r_{m} \pi_{m, 0}^{*} \omega_{0}-c_{1}\left(\left[D_{m}^{\prime}\right]\right)
$$

The forms

$$
\begin{aligned}
\psi_{j} & =\sum_{i=j}^{m-1} \pi_{m, i}^{*} \omega_{i}+\omega_{m} \\
& =\left(\sum_{i=j}^{m} r_{i}\right) \pi_{m, 0}^{*} \omega_{0}-\sum_{i=j}^{m} c_{1}\left(\left[D_{i}^{\prime}\right]\right)
\end{aligned}
$$


are all positive because $\omega_{m}$ is. Let

$$
R_{j}=\sum_{i=j}^{m} r_{i} \quad \text { and } \quad \mathcal{D}_{j}=\sum_{i=j}^{m} D_{i}^{\prime}
$$

and let $H_{j}$ be the induced metric on the line bundle $\left[\mathcal{D}_{j}\right]$. Then

$$
\psi_{j}=R_{j} \pi_{m, 0}^{*} \omega_{0}-c_{1}\left(\left[\mathcal{D}_{j}\right], H_{j}\right)
$$

The form $\psi_{j}$ remains positive if we replace $R_{j}$ by $r>R_{j}$ since $\pi_{m, 0}^{*} \omega_{0} \geq 0$.

The matrix $T(\mathcal{D}, \tilde{E})$ for the divisors $\mathcal{D}_{j}$ in terms of the irreducible divisors $\tilde{E}_{m, k}$ is

$$
T(\mathcal{D}, \tilde{E})=T\left(\mathcal{D}, D^{\prime}\right) T\left(D^{\prime}, E\right) T(E, \tilde{E}) .
$$

The matrix $T\left(\mathcal{D}, D^{\prime}\right)$ is an upper triangular matrix of 1's, the matrix $T\left(D^{\prime}, E\right)$ is a lower triangular matrix of $r_{i k}$ 's which are defined in terms of the integers $l_{j}$ of Theorem (4.3.1), and the matrix $T(E, \tilde{E})$ is the matrix $A$ described in $\S 3.7$. Writing $T(\mathcal{D}, \tilde{E})$ as the product

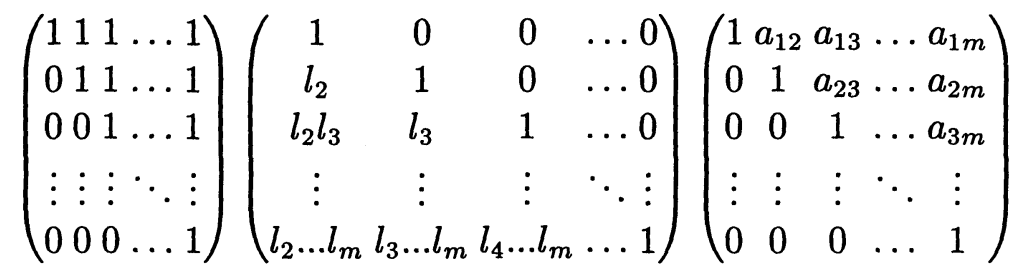

it is easy to see that all entries of $T(\mathcal{D}, \tilde{E})$ are positive and $\operatorname{det} T(\mathcal{D}, \tilde{E})=1$.

$\S 5$. Splitting of a Poincaré-Type $(1,1)$-Form into a Sum of its Two Essential Parts. Let $M$ be a compact complex manifold of dimension $n$ and let $D$ be an effective divisor on $M$ with only normal crossings. In $\S 5.1$ we construct a Poincaré-type $(1,1)$-form $\nu$ on $M-D$ by replacing the expression $|z|^{2}$ in the formula (1.4.1) for the Poincaré form $\omega_{\Delta^{*}}$ by the square of the norm of a section of the line bundle $[D]$. In $\S 5.2$ we study $\nu$ in local coordinates near points of $D$. We may choose coordinates $\left(z_{1}, z_{2}, \ldots, z_{n}\right)$ in which $D$ is given locally by the vanishing of a monomial $z_{1}^{\lambda_{1}} z_{2}^{\lambda_{2}} \ldots z_{k}^{\lambda_{k}}$. The growth of $\nu$ near $D$ may be described in terms of a Chern form 
of $[D]$ and the pullback of $\omega_{\Delta^{*}}$ under the monomial map $\tau$ given by $\tau\left(z_{1}, z_{2}, \ldots, z_{n}\right)=z_{1}^{\lambda_{1}} z_{2}^{\lambda_{2}} \ldots z_{k}^{\lambda_{k}}$. In $\S 5.3$ we describe the quasiisometry classes of sums of forms of the type $\tau^{*} \omega_{\Delta^{*}}$. We use these descriptions in $\S 5.4$ to show that Poincaré and modified Poincaré metrics may be constructed by adding Poincaré-type forms $\nu_{i}$ to multiples of the fundamental form $\omega$ of a hermitian metric on $M$. If $\omega$ is Kähler, the resulting metrics are also Kähler. We conclude $\S 5$ by describing the relationship between modified Saper and modified Poincaré metrics.

(5.1) Definition and decomposition of Poincaré-type $(1,1)$ forms. An effective divisor $D$ on $M$, with only normal crossings, may be expressed as $D=\sum_{i=1}^{m} \lambda_{i} E_{i}$, where $E_{1}, E_{2}, \ldots, E_{m}$ are smooth, reduced, irreducible divisors on $M$ which simultaneously have only normal crossings, and $\lambda_{1}, \lambda_{2}, \ldots, \lambda_{m}$ are positive integers. Let $L=$ $[D]$ be the line bundle on $M$ associated with $D$ and let $h$ be a hermitian metric on $L$. Let $s: M \rightarrow L$ be a global holomorphic section of $L$ such that $(s)=D$. Such a section always exists since $D$ is effective. We denote by $\|s\|$ the norm of $s$ under the metric $h$. Since $M$ is compact, we may also choose $s$ so that $\|s\|<1$ everywhere on $M$. We define on $M-D$ a Poincaré-type $(1,1)$-form $\nu$ associated with the divisor $D$, the section $s$, and the metric $h$ by

$$
\nu=-\frac{\sqrt{-1}}{2 \pi} \partial \bar{\partial} \log \left(\log \|s\|^{2}\right)^{2}
$$

Note that $\log \|s\|^{2} \neq 0$ on $M-D$ and $\log \|s\|^{2} \rightarrow-\infty$ as we approach $D$. Let

$$
\beta=-\log \|s\|^{2}
$$

on $M-D$. We may decompose $\nu$ as $\nu=\mu+\eta$ where

$$
\mu=-\frac{\sqrt{-1}}{\pi} \frac{\partial \bar{\partial} \beta}{\beta} \text { and } \quad \eta=\frac{\sqrt{-1}}{\pi} \frac{\partial \beta \wedge \bar{\partial} \beta}{\beta^{2}} .
$$

Recall from $\S 3.5$ that $c_{1}(L, h)=-\frac{\sqrt{-1}}{2 \pi} \partial \bar{\partial} \log \|s\|^{2}$. Then

$$
\mu=-\frac{2}{\beta} c_{1}(L, h) \text {. }
$$

The Chern form $c_{1}(L, h)$ depends on $h$ but is independent of the choice of section $s$ of $L$. The properties of $\eta$ will be explored in 
Sections 5.2 and 5.3.

If $s^{\prime}$ is another global holomorphic section of $L$ such that $\left(s^{\prime}\right)=D$ and $\left\|s^{\prime}\right\|<1$, then $s^{\prime}=c s$ for some positive constant $c$. Let $\beta^{\prime}=-\log \left\|s^{\prime}\right\|^{2}$. Then $\beta^{\prime}=\beta-2 \log c, \partial \beta^{\prime}=\partial \beta$, and $\bar{\partial} \beta^{\prime}=\bar{\partial} \beta$. From these relationships and our decomposition of $\nu$, we see that although $\nu$ is not independent of $s$, the order of growth of $\nu$ near $D$ is the same for all sections $s$ of $L$ for which $(s)=D$ and $\|s\|<1$.

Note that the form $\mu$ is dominated by any positive $C^{\infty}(1,1)$-form on $M$. Hence

LEMMA 5.1.4. If $\omega$ is the fundamental form of a hermitian metric on $M$ and $\nu$ is a Poincaré-type $(1,1)$-form with the decomposition $\nu=\mu+\eta$ of (5.1.2) then

$$
l \omega+\nu \sim l \omega+\eta
$$

for all sufficiently large integers $l$.

(5.2) Poincaré-type (1,1)-forms in local coordinates. We wish to describe the Poincaré-type $(1,1)$-form $\nu$ in local coordinates near points of $D=\sum_{i=1}^{m} \lambda_{i} E_{i}$. In particular, we wish to examine the growth of the form $\eta$ in the decomposition $\nu=\mu+\eta$ of (5.1.2) and compare it to the growth of the Poincaré form $\omega_{\Delta^{*}}$ on the punctured disc.

Let $q$ be a point in $M$ at which $k$ of the components $E_{i}$ of $D$, say $E_{1}, E_{2}, \ldots, E_{k}$, intersect. Since the collection $\left\{E_{i}\right\}$ has normal crossings, there exist local coordinates $z_{1}, \ldots, z_{n}$ in a neighbourhood $U$ of $q$, such that $E_{i}$ is given locally by the equation $z_{i}=0$ for $i=1, \ldots, k$ and such that $E_{i}$ does not intersect $\bar{U}$ for $i>k$. Recall that we call $\left(z_{1}, \ldots, z_{n}\right)$ normal coordinates for $E_{1}, \ldots, E_{k}$. Locally, $D$ is given by the equation $z_{1}^{\lambda_{1}} z_{2}^{\lambda_{2}} \ldots z_{k}^{\lambda_{k}}=0$, where $\lambda_{i}$ is the multiplicity of $E_{i}$ in $D$. We will use the notation $z=\left(z_{1}, z_{2}, \ldots, z_{n}\right)$ and $z^{\Lambda}=$ $z_{1}^{\lambda_{1}} z_{2}^{\lambda_{2}} \ldots z_{k}^{\lambda_{k}}$. The norm of $s$ is given in local coordinates by $\|s\|^{2}=$ $\left|z^{\Lambda}\right|^{2} g$ for some bounded positive $C^{\infty}$ function $g$ on $U$, and the function $\beta=-\log \|s\|^{2}$ is given locally by

$$
\beta=-\log \left(\left|z^{\Lambda}\right|^{2} g\right) \text {. }
$$

For the rest of $\S 5.2$ we will assume only that $\beta$ is a locally defined function of the form of (5.2.1) where $g$ is some bounded positive $C^{\infty}$ function on $U$. 
Let $\tau$ be the monomial map $\tau: U \rightarrow \Delta$ given by $\tau\left(z_{1}, \ldots, z_{n}\right)=z^{\Lambda}$. We wish to compare the $(1,1)$-form $\eta=\frac{\sqrt{-1}}{\pi} \frac{\partial \beta \wedge \bar{\partial} \beta}{\beta^{2}}$ to the pullback of the Poincare form $\omega_{\Delta^{*}}$ under the map $\tau$. The pullback is

$$
\begin{aligned}
\tau^{*} \omega_{\Delta^{*}} & =\frac{\sqrt{-1}}{\pi} \frac{d\left(z^{\Lambda}\right) \wedge d\left(\bar{z}^{\Lambda}\right)}{\left|z^{\Lambda}\right|^{2}\left(\log \left|z^{\Lambda}\right|^{2}\right)^{2}} \\
& =\frac{\sqrt{-1}}{\pi} \frac{1}{\left(\log \left|z^{\Lambda}\right|^{2}\right)^{2}} \sum_{i, j=1}^{k} \frac{\lambda_{i} \lambda_{j}}{z_{i} \bar{z}_{j}} d z_{i} \wedge d \bar{z}_{j} \\
& \sim \frac{\sqrt{-1}}{\pi} \frac{1}{\beta^{2}} \sum_{i, j=1}^{k} \frac{\lambda_{i} \lambda_{j}}{z_{i} \bar{z}_{j}} d z_{i} \wedge d \bar{z}_{j} .
\end{aligned}
$$

Using the expansion $\beta=-\log z^{\Lambda}-\log \bar{z}^{\Lambda}-\log g$, we express $\eta$ in local coordinates as

$$
\begin{aligned}
\eta= & \frac{\sqrt{-1}}{\pi} \frac{1}{\beta^{2}}\left(\frac{d\left(z^{\Lambda}\right)}{z^{\Lambda}}+\frac{\partial g}{g}\right) \wedge\left(\frac{d\left(\bar{z}^{\Lambda}\right)}{\bar{z}^{\Lambda}}+\frac{\bar{\partial} g}{g}\right) \\
= & \frac{\sqrt{-1}}{\pi}\left(\frac{d\left(z^{\Lambda}\right) \wedge d\left(\bar{z}^{\Lambda}\right)}{\left|z^{\Lambda}\right|^{2}\left(\log \left|z^{\Lambda}\right|^{2} g\right)^{2}}+\frac{d\left(z^{\Lambda}\right) \wedge \bar{\partial} g}{\beta^{2} z^{\Lambda} g}\right. \\
& \left.+\frac{\partial g \wedge d\left(\bar{z}^{\Lambda}\right)}{\beta^{2} g \bar{z}^{\Lambda}}+\frac{\partial g \wedge \bar{\partial} g}{\beta^{2} g^{2}}\right) \\
\sim & \tau^{*} \omega_{\Delta^{*}}+\frac{\sqrt{-1}}{\pi}\left(\sum _ { i = 1 } ^ { k } \sum _ { j = 1 } ^ { n } \left(o\left(\frac{\lambda_{i}}{z_{i} \beta^{2}}\right) d z_{i} \wedge d \bar{z}_{j}\right.\right. \\
& \left.\left.+o\left(\frac{\lambda_{i}}{\bar{z}_{i} \beta^{2}}\right) d z_{j} \wedge d \bar{z}_{i}\right)+\frac{\partial g \wedge \bar{\partial} g}{\beta^{2} g^{2}}\right) .
\end{aligned}
$$

Let $\omega$ be the Euclidean $(1,1)$-form

$$
\omega=\frac{\sqrt{-1}}{\pi} \sum_{i=1}^{n} d z_{i} \wedge d \bar{z}_{i}
$$

We now show that, locally, when we add to $\eta$ any positive multiple of $\frac{1}{\beta} \omega$, all terms but $\tau^{*} \omega_{\Delta^{*}}$ in the expansion of $\eta$ may be ignored. The following lemma is valid for any expression of the form $\eta=\frac{\sqrt{-1}}{\pi} \frac{\partial \beta \wedge \bar{\partial} \beta}{\beta^{2}}$ 
where $\beta=-\log \left(\left|z^{\Lambda}\right|^{2} g\right)$ and $g$ is a bounded positive $C^{\infty}$ function on $U$.

Proposition 5.2.4. Let $a$ be any positive constant. Then the $(1,1)$-form $\frac{a}{\beta} \omega+\eta$ is positive and

$$
\frac{a}{\beta} \omega+\eta \sim \frac{1}{\beta} \omega+\tau^{*} \omega_{\Delta^{*}}
$$

for $z_{1}, z_{2}, \ldots, z_{k}$ close enough (but not equal) to 0 .

Proof. Clearly the term $\frac{\sqrt{-1}}{\pi} \frac{\partial g \wedge \bar{\partial} g}{\beta^{2} g^{2}}$ in the expression (5.2.3) for $\eta$ is dominated by $\frac{a}{\beta} \omega$ for $z_{1}, z_{2}, \ldots, z_{k}$ close enough to 0 , since $\frac{1}{\beta} \rightarrow 0$ as $\left(z_{1}, z_{2}, \ldots, z_{k}\right) \rightarrow(0,0, \ldots, 0)$. Next consider terms of type $o\left(\frac{\lambda_{i}}{z_{i} \beta^{2}}\right) d z_{i} \wedge$ $d \bar{z}_{j}$ and $o\left(\frac{\lambda_{i}}{\bar{z}_{i} \beta^{2}}\right) d z_{j} \wedge d \bar{z}_{i}$ in $\eta$. If $i=j$ these terms are dominated by the corresponding terms of $\tau^{*} \omega_{\Delta^{*}}$. If $i \neq j$, we compare these terms to the $d z_{i} \wedge d \bar{z}_{i}$ term of $\tau^{*} \omega_{\Delta^{*}}$ and the $d z_{j} \wedge d \bar{z}_{j}$ term of $\frac{a}{\beta} \omega$. It is convenient to write these terms from the expansion of $\frac{a}{\beta} \omega+\eta$ in the form of a chart, showing the matrix of coefficients:

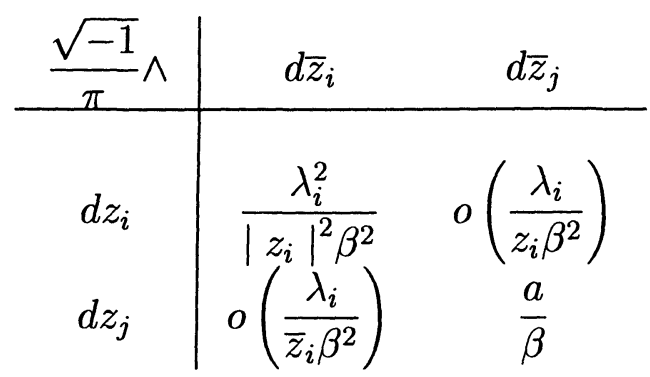

Factoring out powers of $z_{i}, \bar{z}_{i}$, and $\beta$ we obtain

\begin{tabular}{c|cc}
$\frac{\sqrt{-1}}{\pi} \wedge$ & $\frac{1}{\bar{z}_{i} \beta} d \bar{z}_{i}$ & $\frac{1}{\sqrt{\beta}} d \bar{z}_{j}$ \\
\hline$\frac{1}{z_{i} \beta} d z_{i}$ & $\lambda_{i}^{2}$ & $\lambda_{i} o\left(\frac{1}{\sqrt{\beta}}\right)$ \\
$\frac{1}{\sqrt{\beta}} d z_{j}$ & $\lambda_{i} o\left(\frac{1}{\sqrt{\beta}}\right)$ & $a$
\end{tabular}




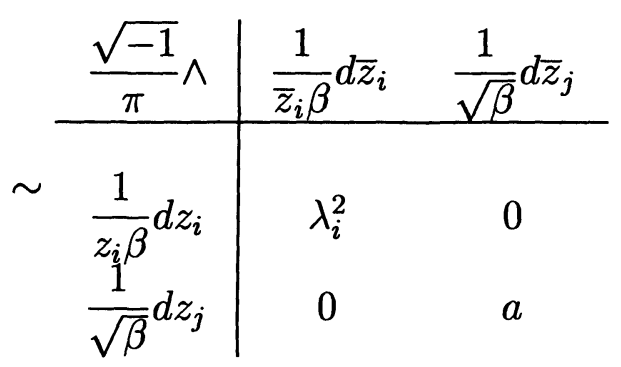

for $z_{1}, z_{2}, \ldots, z_{k}$ close enough to 0 . Then $\frac{a}{\beta} \omega+\eta \sim \frac{a}{\beta} \omega+\tau^{*} \omega_{\Delta^{*}}$ for $z_{1}, z_{2}, \ldots, z_{k}$ close enough to 0 . Since $\omega>0$ and $\tau^{*} \omega_{\Delta^{*}} \geq 0$, we may replace $\frac{a}{\beta} \omega$ by $\frac{1}{\beta} \omega$ and preserve quasi-isometry.

An easy consequence of Proposition (5.2.4) is the following corollary which states that the local quasi-isometry class of a modified Poincaré metric looks the same in every system of normal coordinates. Recall (Definition (1.6.1)) that a metric on $M-D$ with fundamental form $\omega_{P}$ is called a modified Poincaré metric if $\omega_{P}$ has the following property: near each point $q \in M$ at which $k$ components of $D$ intersect, there exist normal coordinates $\left(z_{1}, \ldots, z_{n}\right)$ and nonconstant monomial maps $\tau_{1}, \ldots, \tau_{m}$ of the form $\tau_{i}\left(z_{1}, \ldots, z_{n}\right)=z_{1}^{\lambda_{i 1}} z_{2}^{\lambda_{i 2}} \ldots z_{k}^{\lambda_{i k}}$ such that the matrix $\left(\lambda_{i j}\right)$ has nonnegative integer entries and at least one positive entry in each row and column, and such that locally

$$
\omega_{P} \sim \sum_{i=1}^{m} \tau_{i}^{*} \omega_{\Delta^{*}}+\frac{\sqrt{-1}}{\pi} \sum_{i=1}^{n} d z_{i} \wedge d \bar{z}_{i}
$$

Given such a matrix $\left(\lambda_{i j}\right)$ and any other system of normal coordinates $\left(y_{1}, \ldots, y_{n}\right)$ in a neighbourhood of $q$, let $T_{1}, \ldots, T_{m}$ be the monomial maps given by $T_{i}\left(y_{1}, \ldots, y_{n}\right)=y_{1}^{\lambda_{i 1}} y_{2}^{\lambda_{i 2}} \ldots y_{k}^{\lambda_{i k}}$.

Corollary 5.2.6. If $\omega_{P}$ is the fundamental form of a modified Poincaré metric on $M$ whose quasi-isometry class is given locally in normal coordinates $\left(z_{1}, \ldots, z_{n}\right)$ by (5.2.5), and if $\left(y_{1}, \ldots, y_{n}\right)$ is any other system of normal coordinates, then

$$
\omega_{P} \sim \sum_{i=1}^{m} T_{i}^{*} \omega_{\Delta^{*}}+\frac{\sqrt{-1}}{\pi} \sum_{i=1}^{n} d y_{i} \wedge d \bar{y}_{i}
$$


Proof. The Euclidean form in $y$ is locally quasi-isometric to the Euclidean form $\omega$ in $z$, i.e.

$$
\frac{\sqrt{-1}}{\pi} \sum_{i=1}^{n} d y_{i} \wedge d \bar{y}_{i} \sim \frac{\sqrt{-1}}{\pi} \sum_{i=1}^{n} d z_{i} \wedge d \bar{z}_{i}=\omega
$$

so to prove the corollary it is sufficient to show that $\omega+\tau_{i}^{*} \omega_{\Delta^{*}} \sim$ $\omega+T_{i}^{*} \omega_{\Delta^{*}}$ for all $i$. Since $y$ and $z$ are normal coordinates, there exist bounded nonvanishing holomorphic functions $f_{i}$ such that $z_{i}=y_{i} f_{i}$ for $1 \leq i \leq k$. Let $\beta_{i}=-\log \left|z^{\Lambda_{i}}\right|^{2}$. Then

$$
\beta_{i}=-\log \left|y^{\Lambda_{i}} f^{\Lambda_{i}}\right|^{2}
$$

where the function $g_{i}=\left|f^{\Lambda_{i}}\right|^{2}=\left|f_{1}^{\lambda_{i 1}} f_{2}^{\lambda_{i 2}} \ldots f_{k}^{\lambda_{i k}}\right|^{2}$ is bounded, positive, and $C^{\infty}$. Let

$$
\eta_{i}=\frac{\sqrt{-1}}{\pi} \frac{\partial \beta_{i} \wedge \bar{\partial} \beta_{i}}{\beta_{i}^{2}}
$$

i.e. $\eta_{i}=\tau_{i}^{*} \omega_{\Delta^{*}}$. By Proposition (5.2.4) and quasi-isometry (5.2.7),

$$
\frac{1}{\beta_{i}} \omega+\eta_{i} \sim \frac{1}{\beta_{i}} \omega+T_{i}^{*} \omega_{\Delta^{*}}
$$

But $\omega \geq \frac{1}{\beta_{i}} \omega$ near $D$, so we have $\omega+\tau_{i}^{*} \omega_{\Delta^{*}}=\omega+\eta_{i} \sim \omega+$ $T_{i}^{*} \omega_{\Delta^{*}}$

Similarly, we will show in Corollary (5.3.5) that the local quasiisometry class of a Saper distinguished metric (1.7.1) looks the same in any system of normal coordinates.

(5.3) Pullbacks of Poincaré-type forms under monomial maps. When constructing modified Saper metrics, we will use $(1,1)$-forms of the type $\frac{1}{\beta} \omega+\eta$. Proposition (5.2.4) allows us to replace $\eta$ by a pullback $\tau^{*} \omega_{\Delta^{*}}$ of the Poincaré form $\omega_{\Delta^{*}}$ under a monomial map $\tau$. In this section we describe the quasi-isometry classes of sums of such pullbacks. For the purposes of later calculations, in which we consider a collection of divisors $\left\{D_{j}\right\}$, it is useful to consider monomials $z_{1}^{\lambda_{1}} z_{2}^{\lambda_{2}} \ldots z_{k}^{\lambda_{k}}$ in which some of the powers $\lambda_{i}$ may be 0 . 
For $1 \leq i \leq m$ let $\Lambda_{i}=\left(\lambda_{i 1}, \ldots, \lambda_{i k}\right)$ be a vector of nonnegative integers with at least one nonzero entry. Let $z^{\Lambda_{\imath}}=z_{1}^{\lambda_{11}} z_{2}^{\lambda_{i 2}} \ldots z_{k}^{\lambda_{i k}}$ and let $\tau_{i}$ be the monomial map

$$
\tau_{i}: \Delta^{k} \rightarrow \Delta
$$

given by $\tau_{i}\left(z_{1}, \ldots, z_{k}\right)=z^{\Lambda_{i}}$. Let $\beta_{i}=-\log \left|z^{\Lambda_{i}}\right|^{2}$ and let $\phi_{i}=$ $\beta_{i}^{2} \tau_{i}^{*} \omega_{\Delta^{*}}$. Then

$$
\phi_{i}=\frac{\sqrt{-1}}{\pi} \frac{d\left(z^{\Lambda_{i}}\right) \wedge d\left(\bar{z}^{\Lambda_{i}}\right)}{\left|z^{\Lambda_{2}}\right|^{2}}=\frac{\sqrt{-1}}{\pi} \sum_{j=1}^{k} \sum_{l=1}^{k} \frac{\lambda_{i j} \lambda_{i l}}{z_{j} \bar{z}_{l}} d z_{j} \wedge d \bar{z}_{l} .
$$

The Poincaré form $\omega_{\Delta^{*}}$ is positive definite on $\Delta^{*}$ so all the pullbacks $\tau_{i}^{*} \omega_{\Delta^{*}}$ and all the forms $\phi_{i}$ are positive semi-definite on $\left(\Delta^{*}\right)^{k}$. The form

$$
\phi=\frac{\sqrt{-1}}{\pi} \sum_{i=1}^{k} \frac{d z_{i} \wedge d \bar{z}_{i}}{\left|z_{i}\right|^{2}}
$$

is positive definite on $\left(\Delta^{*}\right)^{k}$.

Lemma 5.3.1. If $k$ of the vectors $\Lambda_{i}$, say $\Lambda_{1}, \ldots, \Lambda_{k}$, are linearly independent, then

$$
\sum_{i=1}^{k} C_{i} \phi_{i} \sim \phi>0
$$

for all positive constants $C_{1}, \ldots, C_{k}$ and for $z_{1}, \ldots, z_{k}$ close enough (but not equal) to 0.

Proof. The matrix of $\phi_{i}$ with respect to $\left\{\xi_{j}=\frac{d z_{j}}{z_{j}}\right\}_{j=1}^{k}$ is $A_{i}=\Lambda_{i}^{T} \Lambda_{i}$ which is positive semi-definite. If $\Lambda_{1}, \ldots, \Lambda_{k}$ are linearly independent then the spans of the matrices $A_{1}, \ldots, A_{k}$ are also linearly independent and $\sum_{i=1}^{k} C_{i} A_{i}$ is positive definite for any positive constants $C_{1}, \ldots, C_{k}$. This implies that $\sum_{i=1}^{k} C_{i} \phi_{i} \sim \phi$ since the matrix of $\phi$ with respect to $\left\{\xi_{j}\right\}$ is $I$.

It follows easily from Lemma (5.3.1) that

LEMMA 5.3 .2 .

i. $\phi \simeq \phi_{i}$ for each $1 \leq i \leq m$ and for $z_{1}, \ldots, z_{k}$ close enough (but not equal) to 0 . 
ii. If the matrix $\left(\lambda_{i j}\right)$ has rank $k$ then $\sum_{i=1}^{m} C_{i} \phi_{i} \sim \phi>0$ for all positive constants $C_{1}, \ldots, C_{m}$ and for $z_{1}, \ldots, z_{k}$ close enough (but not equal) to 0 .

Next we show that a sum of pullbacks of the Poincaré form $\omega_{\Delta^{*}}$ under monomial maps is bounded below by the homogeneous Poincaré form

$$
\psi_{h}=\frac{\sqrt{-1}}{\pi} \frac{1}{\left(\log \left|z_{1} z_{2} \ldots z_{k}\right|^{2}\right)^{2}} \sum_{i=1}^{k} \frac{d z_{i} \wedge d \bar{z}_{i}}{\left|z_{i}\right|^{2}} .
$$

LEMMA 5.3.3. If $k$ of the vectors $\Lambda_{i}$, say $\Lambda_{1}, \ldots, \Lambda_{k}$, are linearly independent then

$$
\sum_{i=1}^{m} C_{i} \tau_{i}^{*} \omega_{\Delta^{*}} \stackrel{\sim}{\geq} \psi_{h}>0
$$

for all positive constants $C_{1}, \ldots, C_{m}$ and for $z_{1}, \ldots, z_{k}$ close enough (but not equal) to 0 . If, in addition, the integers $\lambda_{i j}$ are all positive then

$$
\sum_{i=1}^{m} C_{i} \tau_{i}^{*} \omega_{\Delta^{*}} \sim \psi_{h}
$$

Proof. Let $\beta=-\log \left|z_{1} z_{2} \ldots z_{k}\right|^{2}$. There exists a positive constant $a$ such that $a \beta \geq \beta_{i}$ for $1 \leq i \leq m$. Then

$$
\sum_{i=1}^{m} C_{i} \tau_{i}^{*} \omega_{\Delta^{*}}=\sum_{i=1}^{m} \frac{C_{i}}{\beta_{i}^{2}} \phi_{i} \geq \frac{1}{a^{2} \beta^{2}} \sum_{i=1}^{m} C_{i} \phi_{i} .
$$

By Lemma (5.3.2) and the definitions of $\beta, \phi$, and $\psi_{h}$,

$$
\frac{1}{a^{2} \beta^{2}} \sum_{i=1}^{m} C_{i} \phi_{i} \sim \frac{1}{\beta^{2}} \phi=\psi_{h}
$$

for $z_{1}, z_{2}, \ldots, z_{k}$ near 0 . This proves part (i). If $\lambda_{i j}>0$ for all $i$ and $j$, then $\beta_{i} \sim \beta$ and $\tau_{i}^{*} \omega_{\Delta^{*}} \sim \frac{1}{\beta^{2}} \phi_{i}$ for all $i$. In this case the inequality $\geq$ in equation (5.3.4) becomes quasi-isometry and we obtain part (ii). 
Recall that a metric on $M-D$ is called a Saper distinguished metric if its fundamental form $\omega_{\text {Sap }}$ may be described locally in normal coordinates by the quasi-isometry

$$
\omega_{\text {Sap }} \sim \psi_{h}+\frac{1}{\beta} \omega_{\text {Eucl }}
$$

where $\omega_{\text {Eucl }}$ is the Euclidean $(1,1)$-form

$$
\omega_{\mathrm{Eucl}}=\frac{\sqrt{-1}}{\pi} \sum_{i=1}^{n} d z_{i} \wedge d \bar{z}_{i} .
$$

A homogeneous Poincaré metric has a fundamental form $\omega_{\mathrm{P}, \text { hom }}$ given locally by

$$
\omega_{\mathrm{P}, \mathrm{hom}} \sim \psi_{h}+\omega_{\mathrm{Eucl}} .
$$

We can now easily show that the local quasi-isometry class of $\omega_{\text {Sap }}$ (respectively $\omega_{\mathrm{P}, \mathrm{hom}}$ ) looks the same in any system of normal coordinates.

COROLlary 5.3.5. The quasi-isometry class of a Saper distinguished metric (respectively a homogeneous Poincaré metric) on $M-D$ is independent of the choice of normal coordinates.

Proof. Use part (ii) of Lemma (5.3.3) and Proposition (5.2.4).

Finally, we show that pullbacks of the Poincaré form $\omega_{\Delta^{*}}$ under monomial maps are bounded above by the Poincaré form

$$
\psi_{P}=\frac{\sqrt{-1}}{\pi} \sum_{i=1}^{k} \frac{d z_{i} \wedge d \bar{z}_{i}}{\left|z_{i}\right|^{2}\left(\log \left|z_{i}\right|^{2}\right)^{2}} .
$$

LEMMA 5.3.6. Let $\Lambda=\left(\lambda_{1}, \lambda_{2}, \ldots, \lambda_{k}\right)$ be a vector of nonnegative integers with at least one nonzero entry and let $\tau$ be the corresponding map $\tau: \Delta^{k} \rightarrow \Delta$ given by $\tau\left(z_{1}, z_{2}, \ldots, z_{k}\right)=z_{1}^{\lambda_{1}} z_{2}^{\lambda_{2}} \ldots z_{k}^{\lambda_{k}}$. Then

$$
\tau^{*} \omega_{\Delta^{*}} \stackrel{\sim}{\leq} \psi_{P}
$$

for $z_{1}, \ldots, z_{k}$ close enough (but not equal) to 0 .

Proof. Let $l$ be the number of entries of $\Lambda$ which are nonzero. Reindex the variables $z_{i}$ so that $\lambda_{i}>0$ for $1 \leq i \leq l$ and $\lambda_{i}=0$ 
otherwise. Let $\beta=-\log \left|z_{1}^{\lambda_{1}} z_{2}^{\lambda_{2}} \ldots z_{l}^{\lambda_{l}}\right|^{2}$. Then $\beta \tilde{\geq}-\log \left|z_{i}\right|^{2}$ for $1 \leq i \leq l$. Let

$$
\phi^{\prime}=\frac{\sqrt{-1}}{\pi} \sum_{i=1}^{l} \frac{d z_{i} \wedge d \bar{z}_{i}}{\left|z_{i}\right|^{2}}
$$

and let $\phi_{\Lambda}=\beta^{2} \tau^{*} \omega_{\Delta^{*}}$. The form $\phi_{\Lambda}$ is positive semi-definite and by Lemma (5.3.2), $\phi_{\Lambda} \stackrel{\sim}{\leq} \phi^{\prime}$. Dividing $\phi_{\Lambda}$ by $\beta^{2}$ and using the indicated lower bounds for $\beta$ we obtain

$$
\begin{aligned}
\tau^{*} \omega_{\Delta^{*}} & \leq \frac{1}{\beta^{2}} \phi^{\prime} \\
& \simeq \frac{\sqrt{-1}}{\pi} \sum_{i=1}^{l} \frac{d z_{i} \wedge d \bar{z}_{i}}{\left|z_{i}\right|^{2}\left(\log \left|z_{i}\right|^{2}\right)^{2}} \\
& \leq \psi_{P} .
\end{aligned}
$$

(5.4) Modified Poincaré metrics. It is easy to construct Poincaré and modified Poincaré metrics on $M-D$ using the results of Sections (5.1) - (5.3). The components $E_{1}, \ldots, E_{m}$ of $D$ are smooth, reduced, irreducible divisors which simultaneously have only normal crossings. Let $D_{1}, \ldots, D_{r}$ be effective divisors of the form

$$
D_{i}=\sum_{j=1}^{m} \lambda_{i j} E_{j}
$$

such that the matrix $\left(\lambda_{i j}\right)$ has nonnegative integer entries and at least one positive entry in each row and column, i.e. the divisors $D_{i}$ are effective and their sum has the same support as $D$. Let $s_{i}: M \rightarrow\left[D_{i}\right]$ be a global holomorphic section of the line bundle $L_{i}=\left[D_{i}\right]$ such that $\left(s_{i}\right)=D_{i}$ and let $h_{i}$ be a hermitian metric on $L_{i}$. Let

$$
\nu_{i}=-\frac{\sqrt{-1}}{2 \pi} \partial \bar{\partial} \log \left(\log \left\|s_{i}\right\|^{2}\right)^{2} .
$$

The following proposition is an easy consequence of Lemmas (5.1.4) and (5.3.3) and Proposition (5.2.4).

Proposition 5.4.1. If $\omega$ is the fundamental form of a hermitian metric on $M$ and $l_{1}, \ldots, l_{r}$ are positive integers, then the form

$$
\omega_{P}=l \omega+\sum_{i=1}^{r} l_{i} \nu_{i}
$$


determines a modified Poincaré metric on the noncompact manifold $M-D$ for all sufficiently large integers $l$. Moreover

i. If $M$ is Kähler and $\omega$ is a Kähler form on $M$ then $\omega_{P}$ is also Kähler.

ii. If $r=m$ and $D_{i}=E_{i}$ for $1 \leq i \leq m$ then the metric determined by $\omega_{P}$ is a true Poincaré metric.

iii. If the matrix $\Lambda=\left(\lambda_{i j}\right)$ satisfies the condition

(*) the entries are all positive and the columns are linearly independent

then the metric determined by $\omega_{P}$ is a homogeneous Poincaré metric. More generally, if $D$ has several connected components, the metric determined by $\omega_{P}$ is a homogeneous Poincaré metric if the submatrix of $\Lambda$ corresponding to each connected component of $D$ satisfies condition $(*)$.

(5.5) Modified Saper metrics. Suppose that $M_{0}$ is a compact complex manifold and $\pi: M \rightarrow M_{0}$ is a holomorphic map whose restriction to $M-D$ is a biholomorphism onto its image. Let $\omega_{0}$ be the fundamental form of a hermitian metric on $M_{0}$. Let $D_{1}, \ldots, D_{r}$ be divisors on $M$ of the type described above in $\S 5.4$ and let $\nu_{1}, \ldots, \nu_{r}$ be Poincaré-type $(1,1)$-forms for these divisors. Let $l_{0}, l_{1}, \ldots, l_{r}$ be positive integers. Recall from $\S 1.8$ that if the modified Saper form

$$
\omega_{S}=l_{0} \pi^{*} \omega_{0}+\sum_{i=1}^{r} l_{i} \nu_{i}
$$

is positive then we say it determines a modified Saper metric which is distinguished with respect to $\pi$. If in addition $\omega_{0}$ is Kähler, then so is $\omega_{S}$.

Let $\omega$ be the fundamental form of a hermitian metric on $M$, as above. Note that any positive $C^{\infty}(1,1)$-form on $M$ dominates $\pi^{*} \omega_{0}$. The next result follows immediately from Proposition (5.4.1).

Proposition 5.5.1. If $\omega$ is the fundamental form of a hermitian metric on $M$ and $\omega_{S}$ is a modified Saper form on $M-D$, then the form

$$
\omega_{P}=\omega_{S}+l \omega
$$

determines a modified Poincaré metric on $M-D$ for all sufficiently 
large integers $l$. Moreover if $\omega_{S}$ is positive we may use $l=1$ and we have

i. If $\omega_{S}$ is homogeneous so is $\omega_{P}$.

ii. If $M_{0}$ and $M$ are Kähler and if $\omega_{0}$ and $\omega$ are Kähler forms, then $\omega_{S}$ and $\omega_{P}$ are also Kähler.

In $\S 7$ we construct Kähler modified Saper metrics, distinguished with respect to a single blow-up of a compact Kähler manifold $M$. In $§ 8$ we construct Kähler modified Saper metrics inductively on successive blow-ups and exhibit in local coordinates the relationship of each new metric to the pullback of the previous. When each centre is either contained in the total exceptional divisor of the previous blow-ups or disjoint from it, these metrics are homogeneous. When the image of $D$ in $M$ is of dimension 0 , the metrics are exactly Saper distinguished metrics. In $\S 9$ we construct Kähler homogeneous Saper metrics without the restriction that each centre lie in the previous total exceptional divisor.

$\S 6$. Main ingredient of completeness of our metrics. Let $M$ be a compact Kähler manifold and let $D$ be an effective divisor on $M$ with only normal crossings. In this section we show that if a metric on $M-D$ is bounded below locally, near $D$, by pullbacks of the Poincaré metric on the punctured disc under appropriate monomial maps, then the metric is complete. In particular, modified Poincaré metrics are complete. When we construct our modified Saper metrics in Sections 7, 8, and 9, we will use Proposition (5.2.4) and the results of this section to show that they are also complete.

We first consider the case in which a single monomial map suffices.

Proposition 6.1.1. Let $\omega_{M-D}$ be a Kähler form on $M-D$. Suppose that $\omega_{M-D}$ satisfies the following condition near each point $q \in D$ : there exist normal coordinates $z_{1}, \ldots, z_{n}$ on a neighbourhood $U$ of $q$ such that the components of $D$ are given locally by the equations $z_{i}=0$ for $i=1, \ldots, k$, and there exists a monomial map $\tau: U \rightarrow \Delta$ given by

$$
\tau\left(z_{1}, \ldots, z_{n}\right)=z_{1}^{\lambda_{1}} \ldots z_{k}^{\lambda_{k}}
$$

for some positive integers $\lambda_{1}, \ldots, \lambda_{k}$, such that

$$
\omega_{M-D} \geq \tau^{*} \omega_{\Delta^{*}}
$$


on $U$. Then the metric on $M-D$ determined by $\omega_{M-D}$ is complete.

Proof. We will use the completeness of the Poincaré metric on the punctured disc $\Delta^{*}$. We are only concerned with the behaviour of this metric near $0 \in \Delta$.

Let $x$ and $y$ be points in $M$ and let $d_{M}(x, y)$ be the distance between them in the metric determined by some Kähler form $\omega_{M}$ on all of $M$. Similarly, for $x, y \in M-D$, let $d_{M-D}(x, y)$ be the distance between $x$ and $y$ in the metric determined by $\omega_{M-D}$. Let $\left\{x_{n}\right\}$ be a sequence in $M-D$ which is Cauchy with respect to $d_{M-D}$. Since $M$ is compact, there is a subsequence $\left\{x_{n_{i}}\right\}$ of $\left\{x_{n}\right\}$ which converges with respect to $d_{M}$ to a point $q \in M$.

Suppose that $q \in M-D$. Then there exists a neighbourhood $U$ of $q$ in $M-D$ containing all but finitely many of the points $\left\{x_{n_{i}}\right\}$ and such that $\omega_{M}$ and $\omega_{M-D}$ are quasi-isometric on $U$. Then $\left\{x_{n_{i}}\right\}$ converges to $q$ in the metric determined by $\omega_{M-D}$ and so $\left\{x_{n}\right\}$ also converges to $q$ in this metric.

Now suppose that $q \in D$ and let $U$ be a coordinate neighbourhood of $q$ in $M$ on which there is a monomial map $\tau$ of the form described above. Let $d^{*}$ be the Poincaré distance function on $\Delta^{*}$. After shrinking $U$ if necessary, there is a positive constant $c$ such that for any $x, y \in U-U \cap D$ we have

$$
d_{M-D}(x, y) \geq c d^{*}(\tau(x), \tau(y))
$$

Similarly, if $d$ is the usual Euclidean distance function on $\Delta$ then there is a positive constant $C$ such that for any $x, y \in U$ we have

$$
d_{M}(x, y) \geq C d(\tau(x), \tau(y)) .
$$

Since the sequence $\left\{x_{n_{i}}\right\}$ is Cauchy with respect to $d_{M-D}$, the sequence $\left\{\tau\left(x_{n_{i}}\right)\right\}$ is Cauchy with respect to $d^{*}$. Therefore $\left\{\tau\left(x_{n_{i}}\right)\right\}$ converges to a point $p \in \Delta^{*}$. Similarly, since the sequence $\left\{x_{n_{i}}\right\}$ converges with respect to $d_{M}$ to $q \in D$, the sequence $\left\{\tau\left(x_{n_{i}}\right)\right\}$ converges to $0 \in \Delta$ with respect to the usual distance function $d$ on $\Delta$. This is impossible, since in a neighbourhood of $p \in \Delta^{*}$ the distance functions $d$ and $d^{*}$ are quasi-isometric. Therefore $q \in M-D$ and the metric determined by $\omega_{M-D}$ is complete.

A similar result applies to a collection of monomial maps: 
Proposition 6.1.2. Let $\omega_{M-D}$ be a Kähler form on $M-D$. Suppose that $\omega_{M-D}$ satisfies the following condition near each point $q \in D$ : there exist normal coordinates $z_{1}, \ldots, z_{n}$ on a neighbourhood $U$ of $q$ such that the components of $D$ are given locally by the equations $z_{i}=0$ for $i=1, \ldots, k$, and there exist a monomial maps $\tau_{1}, \ldots, \tau_{r}$ from $U$ to $\Delta$ of the form

$$
\tau_{i}\left(z_{1}, \ldots, z_{n}\right)=z_{1}^{\lambda_{i 1}} \ldots z_{k}^{\lambda_{i k}}
$$

such that the matrix $\left(\lambda_{i j}\right)$ has nonnegative integer entries and at least one positive entry in each row and column, and such that

$$
\omega_{M-D} \widetilde{\geq} \sum_{i=1}^{r} \tau_{i}^{*} \omega_{\Delta^{*}}
$$

on $U$. Then the metric on $M-D$ determined by $\omega_{M-D}$ is complete.

Proof. We proceed as before, replacing the map $\tau: U \rightarrow \Delta$ of the previous proposition by the map

$$
\tau=\left(\tau_{1}, \ldots, \tau_{r}\right): U \rightarrow \Delta^{r}
$$

and replacing the Poincaré form $\omega_{\Delta^{*}}$ on $\Delta^{*}$ by a product of Poincaré forms $\omega_{\left(\Delta^{*}\right)^{r}}$ on $\left(\Delta^{*}\right)^{r}$. Note that $\omega_{\left(\Delta^{*}\right)^{r}}$ determines a complete metric on $\left(\Delta^{*}\right)^{r}$ and that

$$
\tau^{*} \omega_{\left(\Delta^{*}\right)^{r}}=\sum_{i=1}^{r} \tau_{i}^{*} \omega_{\Delta^{*}}
$$

We complete the proof in the same way as above.

The next corollary follows immediately from the definition of modified Poincaré metrics.

Corollary 6.1.3. Modified Poincaré metrics are complete.

When we construct our modified Saper metrics we will show that they also have the required lower bound. 


\section{§7. Modified Saper and Poincaré metrics for a single blow-} up.

(7.1) Introduction. Let $M$ be a compact Kähler manifold with Kähler form $\omega$. Let $\pi: \tilde{M} \rightarrow M$ be the blow-up of $M$ along a submanifold $C \subset M$, with exceptional divisor $E=\pi^{-1}(C)$ and associated line bundle $L=[E]$ on $\tilde{M}$. Let $h$ be a hermitian metric on $L$ with the property that the $(1,1)$-form

$$
\tilde{\omega}=l \pi^{*} \omega-c_{1}(L, h)
$$

on $\tilde{M}$ is positive and consequently Kähler for all sufficiently large integers $l$. In $\S 4$ we showed that such metrics $h$ exist. The Chern form $c_{1}(L, h)$ may be written as

$$
c_{1}(L, h)=-\frac{\sqrt{-1}}{2 \pi} \partial \bar{\partial} \log \|s\|^{2}
$$

where $s: \tilde{M} \rightarrow L$ is a global holomorphic section of $L$ such that $(s)=E$ and such that the norm $\|s\|$ of $s$ under the metric $h$ satisfies $\|s\|<1$ everywhere on $\tilde{M}$. The Kähler form $\tilde{\omega}$ determines an incomplete metric on $\tilde{M}-E \cong M-C$ and the completion of $M-C$ with respect to this metric is $\tilde{M}$.

We now define a Poincaré-type $(1,1)$-form $\nu$ on $\tilde{M}-E$ by the equation

$$
\nu=-\frac{\sqrt{-1}}{2 \pi} \partial \bar{\partial} \log \left(\log \|s\|^{2}\right)^{2}
$$

We will show in this section that the $(1,1)$-form

$$
\omega_{S}=l \pi^{*} \omega+\nu
$$

on $\tilde{M}-E$ is positive and hence Kähler for all sufficiently large integers $l$. The corresponding Kähler metric is a complete modified Saper metric, distinguished with respect to the blow-up map $\pi$. When $\operatorname{dim} C=0$, i.e. when $\tilde{M}$ is the blow-up of an isolated point in $M$, our modified Saper metric is precisely a Saper distinguished metric.

It follows from Proposition (5.5.1) that the $(1,1)$-form

$$
\omega_{P}=\omega_{S}+\tilde{\omega}
$$


is the Kähler form of a Poincaré metric on $\tilde{M}-E$, i.e. the sum of our modified Saper metric on $\tilde{M}-E$ and the restriction to $\tilde{M}-E$ of a Kähler metric on $\tilde{M}$ is Poincaré. We may replace $\tilde{\omega}$ by any other Kähler form on $\tilde{M}$ since all Kähler metrics on a compact manifold are quasi-isometric.

We proved in $\S 6$ that Poincaré metrics are complete. Completeness of our modified Saper metric follows from the local description of the quasi-isometry class of $\omega_{S}$ and Proposition (6.1.1).

The constructions which we will give in $\S \S 8-9$ of modified Saper and Poincaré metrics for successive blow-ups $M_{j} \rightarrow M_{j-1}$ are similar, but the estimates used are more delicate.

(7.2) Main results. Let $M$ and $\tilde{M}$ be as above and let $\omega_{\Delta^{*}}$ be the Poincaré form on the punctured disc. For any point $q \in E$, we may choose local coordinates $\left(z_{1}, \ldots, z_{n}\right)$ on a neighbourhood $U$ of $q$ and $\left(Z_{1}, \ldots, Z_{n}\right)$ on a neighbourhood of $\pi(q) \in C$ such that

i. $E$ is given locally by the equation $z_{1}=0$,

ii. $C$ is given locally by the equations $Z_{1}=\ldots=Z_{k}=0$, and

iii. $\pi$ is given locally by the equations:

$$
\begin{array}{ll}
Z_{1}=z_{1} & \\
Z_{i}=z_{1} z_{i} & \text { for } \quad 2 \leq i \leq k \\
Z_{i}=z_{i} & \text { for } \quad k+1 \leq i \leq n
\end{array}
$$

We will call the coordinates $\left(z_{1}, \ldots, z_{n}\right)$ normal blow-up coordinates for $\pi$ corresponding to $\left(Z_{1}, \ldots, Z_{n}\right)$. Let $\tau: U \rightarrow \Delta$ be the monomial map given by $\tau\left(z_{1}, \ldots, z_{n}\right)=z_{1}$.

Proposition 7.2.1. (Modified Saper metrics for a single blowup.) The (1,1)-form

$$
\omega_{S}=l \pi^{*} \omega+\nu
$$

determines a complete Kähler metric on $\tilde{M}-E$ for all sufficiently large integers $l$. This metric is a modified Saper metric which is distinguished with respect to the map $\pi$. If $q \in E$ and if $\left(z_{1}, \ldots, z_{n}\right)$ are normal blow-up coordinates on a neighbourhood $U$ of $q$ then $\left.\omega_{S}\right|_{U}$ 
is locally quasi-isometric to all of the following:

(i)

$$
\left.\pi^{*} \omega\right|_{U}+\frac{\sqrt{-1}}{\pi} \frac{1}{\left.|\log | z_{1}\right|^{2} \mid} \sum_{i=2}^{k} d z_{i} \wedge d \bar{z}_{i}+\tau^{*} \omega_{\Delta^{*}}
$$

$$
\left.\pi^{*} \omega\right|_{U}+\frac{\sqrt{-1}}{\pi}\left(\frac{1}{\left.|\log | z_{1}\right|^{2} \mid} \sum_{i=1}^{n} d z_{i} \wedge d \bar{z}_{i}+\frac{d z_{1} \wedge d \bar{z}_{1}}{\left|z_{1}\right|^{2}\left(\log \left|z_{1}\right|^{2}\right)^{2}}\right)
$$

$$
\begin{aligned}
& \frac{\sqrt{-1}}{\pi}\left(\frac{d z_{1} \wedge d \bar{z}_{1}}{\left|z_{1}\right|^{2}\left(\log \left|z_{1}\right|^{2}\right)^{2}}+\frac{1}{\left.|\log | z_{1}\right|^{2} \mid} \sum_{i=2}^{k} d z_{i} \wedge d \bar{z}_{i}\right. \\
& \left.\quad+\sum_{i=k+1}^{n} d z_{i} \wedge d \bar{z}_{i}\right) .
\end{aligned}
$$

Note that completeness follows directly from quasi-isometry (i) by Proposition (6.1.1).

Quasi-isometries (i) and (ii) describe the relationship between our modified Saper metric on $\tilde{M}-E$ and the metric on $\tilde{M}-E$ induced from $M$. Quasi-isometry (i) means that locally, near $E$, our modified Saper metric on $\tilde{M}-E$ looks like the metric induced from $M$ plus a small term in the fibre directions plus a term with Poincaré-type growth. We will prove a similar statement for successive blow-ups. Quasi-isometry (ii) means that our modified Saper metric is locally quasi-isometric to the sum of the incomplete metric induced from $M$ and a Saper distinguished metric. In (iii) we describe the quasiisometry class completely in local coordinates. Descriptions (ii) and (iii) of $\omega_{S}$ in local coordinates are more difficult to generalize to successive blow-ups except in special cases, and will be replaced by upper and lower bounds for $\omega_{S}$.

Let $\omega_{\text {Sap }}$ be the fundamental form of a Saper distinguished metric on $\tilde{M}-E$, not necessarily Kähler. As a corollary to the local description (ii) we obtain the following global quasi-isometry.

COROLlaRY 7.2.2. The Kähler modified Saper metric on $\tilde{M}-$ $E$ associated with $\omega_{S}$ is quasi-isometric to the sum of the metric induced from $M$ and a Saper distinguished metric on $\tilde{M}-E$. More concisely:

$$
\omega_{S} \sim \pi^{*} \omega+\omega_{\text {Sap }}
$$


We can say even more in the case $\operatorname{dim} C=0$. In this case, $k=n$ and the last term of (iii) disappears, giving us the following:

COROLlaRY 7.2.3. (Blow-ups of isolated points.) If $\operatorname{dim} C=0$ then $\omega_{S}$ is the fundamental form of a Kähler metric which is distinguished in the sense of Saper.

Given a Kähler form $\omega$ on $M$, we may construct a Kähler form $\tilde{\omega}$ on $\tilde{M}$, by Theorem (4.2.2). In local coordinates $\left(z_{1}, \ldots, z_{n}\right)$ near any point $q \in \tilde{M}$, every positive $C^{\infty}(1,1)$-form on $\tilde{M}$ is locally quasiisometric to the Euclidean form $\frac{\sqrt{-1}}{\pi} \sum_{i=1}^{n} d z_{i} \wedge d \bar{z}_{i}$. Comparison to description (iii) of our modified Saper metric gives us

Corollary 7.2.4. (Kähler Poincaré metrics for a single blowup.) Let

$$
\omega_{P}=\omega_{S}+\tilde{\omega}
$$

where $\tilde{\omega}$ is the Kähler form of a metric on $\tilde{M}$. Then $\omega_{P}$ is a Kähler Poincaré metric on $\tilde{M}-E$ and $\omega_{P}>\omega_{S}$. If $q \in E$ and if $\left(z_{1}, \ldots, z_{n}\right)$ are normal blow-up coordinates on a neighbourhood $U$ of $q$ then

$$
\left.\omega_{P}\right|_{U} \sim \frac{\sqrt{-1}}{\pi}\left(\frac{d z_{1} \wedge d \bar{z}_{1}}{\left|z_{1}\right|^{2}\left(\log \left|z_{1}\right|^{2}\right)^{2}}+\sum_{i=2}^{n} d z_{i} \wedge d \bar{z}_{i}\right) .
$$

The corresponding metrics for successive blow-ups are modified Poincaré metrics.

(7.3) Proof of Proposition 7.2.1. To show that $\omega_{S}$ is a Kähler form, we must show that it is positive, hermitian, and $d$-closed. The form $\nu$ is hermitian and $d$-closed by Lemma (1.2.1), so we need only show that $\omega_{S}$ is positive. Since $\tilde{M}$ is compact, it is enough to show that for each point $q \in \tilde{M}$ there is a neighbourhood $U$ of $q$ on which $l \pi^{*} \omega+\nu$ is positive for $l \gg 0$. This is clear for $q \in \tilde{M}-E$, since in this case there is a neighbourhood $U$ of $q$ on which $\pi^{*} \omega$ is positive and $\nu$ is bounded.

For $q \in E$ we will write $\pi^{*} \omega$ and $\nu$ in normal blow-up coordinates near $q$ and examine the local quasi-isometry class of $\pi^{*} \omega+\nu$. Let $\left(z_{1}, \ldots, z_{n}\right)$ be normal blow-up coordinates in a neighbourhood $U$ of $q$, corresponding to coordinates $\left(Z_{1}, \ldots, Z_{n}\right)$ in a neighbourhood of $\pi(q) \in C$, as in $\S 7.2$. We write $z_{f}=\left(z_{2}, \ldots, z_{k}\right)$ (fibre coordinates 
of the map $E \rightarrow C), Z_{f}=\left(Z_{2}, \ldots, Z_{k}\right), z_{C}=\left(z_{k+1}, \ldots, z_{n}\right)$, and $Z_{C}=\left(Z_{k+1}, \ldots, Z_{n}\right)$ (coordinates on $C$ ). Then $\pi$ is given locally by the equations

$$
Z_{1}=z_{1}, \quad Z_{f}=z_{1} z_{f}, \quad \text { and } \quad Z_{C}=z_{C} .
$$

We will also use the convention that the repeated index $f$ is summed over the set $\{2, \ldots, k\}$ and the repeated index $C$ over the set $\{k+$ $1, \ldots, n\}$. In particular,

$$
d z_{f} \wedge d \bar{z}_{f}=\sum_{i=2}^{k} d z_{i} \wedge d \bar{z}_{i} \text { and } d z_{C} \wedge d \bar{z}_{C}=\sum_{i=k+1}^{n} d z_{i} \wedge d \bar{z}_{i} .
$$

Consider the local expressions for $\omega$ and $\pi^{*} \omega$. Since $\omega$ is positive on $M, \omega$ is locally quasi-isometric to $\frac{\sqrt{-1}}{\pi} \sum_{i=1}^{n} d Z_{i} \wedge d \bar{Z}_{i}$. Then locally

$$
\begin{array}{r}
\pi^{*} \omega \quad \frac{\sqrt{-1}}{\pi}\left(d z_{1} \wedge d \bar{z}_{1}+\left(z_{f} d z_{1}+z_{1} d z_{f}\right) \wedge\left(\bar{z}_{f} d \bar{z}_{1}+\bar{z}_{1} d \bar{z}_{f}\right)\right. \\
\left.+d z_{C} \wedge d \bar{z}_{C}\right) .
\end{array}
$$

Keeping track only of the dominant terms, we write this information in the form of a chart:

$$
\pi^{*} \omega \sim \begin{array}{c|ccc}
\frac{\sqrt{-1}}{\pi} \wedge & d \bar{z}_{1} & d \bar{z}_{f} & d \bar{z}_{C} \\
\hline d z_{1} & 1 & \bar{z}_{1} z_{f} & 0 \\
d z_{f} & z_{1} \bar{z}_{f} & \left|z_{1}\right|^{2} & 0 \\
d z_{C} & 0 & 0 & 1
\end{array}
$$

where the 1 in the $d z_{C} \wedge d \bar{z}_{C}$ spot represents the identity matrix $I_{C}$ and the $\left|z_{1}\right|^{2}$ in the $d z_{f} \wedge d \bar{z}_{f}$ spot represents $\left|z_{1}\right|^{2} I_{f}$.

Next consider the Poincaré-type $(1,1)$-form $\nu=-\frac{\sqrt{-1}}{2 \pi} \partial \bar{\partial} \log \beta^{2}$ where $\beta=-\log \|s\|^{2}$. The local expression for $\beta$ is

$$
\beta=-\log \left(\left|z_{1}\right|^{2} g\right)
$$

where $g$ is a bounded positive $C^{\infty}$ function on $U$, giving the metric $h$ on $L$ locally. We decompose $\nu$ as $\nu=\mu+\eta$, where

$$
\mu=-\frac{\sqrt{-1}}{\pi} \frac{\partial \bar{\partial} \beta}{\beta} \quad \text { and } \quad \eta=\frac{\sqrt{-1}}{\pi} \frac{\partial \beta \wedge \bar{\partial} \beta}{\beta^{2}} .
$$


In the following lemma we describe the quasi-isometry class of $\pi^{*} \omega+\mu$ in local coordinates. Then we will use Proposition (5.2.4) to describe the quasi-isometry class of $\pi^{*} \omega+\nu=\pi^{*} \omega+\mu+\eta$.

LEMMA 7.3.2. The restriction to $U$ of the $(1,1)$-form $\pi^{*} \omega+\mu$ is positive and quasi-isometric to all of the following for $z_{1}$ close enough (but not equal) to 0:

i. $\pi^{*} \omega+\frac{\sqrt{-1}}{\pi} \frac{1}{\beta} \sum_{i=1}^{n} d z_{i} \wedge d \bar{z}_{i}$,

ii. $\frac{\sqrt{-1}}{\pi}\left(d z_{1} \wedge d \bar{z}_{1}+\frac{1}{\beta} d z_{f} \wedge d \bar{z}_{f}+d z_{C} \wedge d \bar{z}_{C}\right)$, and

iii. $\pi^{*} \omega+\frac{\sqrt{-1}}{\pi} \frac{1}{\beta} d z_{f} \wedge d \bar{z}_{f}$.

Proof. Recall from (5.1.3) that $\mu$ may be expressed in terms of a Chern form as

$$
\mu=-\frac{2}{\beta} c_{1}(L, h)
$$

By our choice of metric $h$ on $L$, the Chern form $c_{1}(L, h)$ is negative on the fibres of the map $E \rightarrow C$. The form

$$
\lambda \pi^{*} \omega+\beta \mu=\lambda \pi^{*} \omega-2 c_{1}(L, h)
$$

is positive for large $\lambda$, by Proposition (4.2.1), and is thus locally quasi-isometric to the Euclidean $(1,1)$-form $\frac{\sqrt{-1}}{\pi} \sum_{i=1}^{n} d z_{i} \wedge d \bar{z}_{i}$. Writing $\pi^{*} \omega+\mu$ as

$$
\left(1-\frac{\lambda}{\beta}\right) \pi^{*} \omega+\frac{1}{\beta}\left(\lambda \pi^{*} \omega+\beta \mu\right)
$$

and observing that $\frac{1}{\beta}=-\frac{1}{\log \left(\left|z_{1}\right|^{2} g\right)} \rightarrow 0$ as $z_{1} \rightarrow 0$, we obtain quasiisometry (i).

To prove (ii), we write $\pi^{*} \omega+\mu$ in local coordinates in chart format. Using part (i) and factoring $\frac{1}{\sqrt{\beta}}$ out of the second row and column, 
we obtain

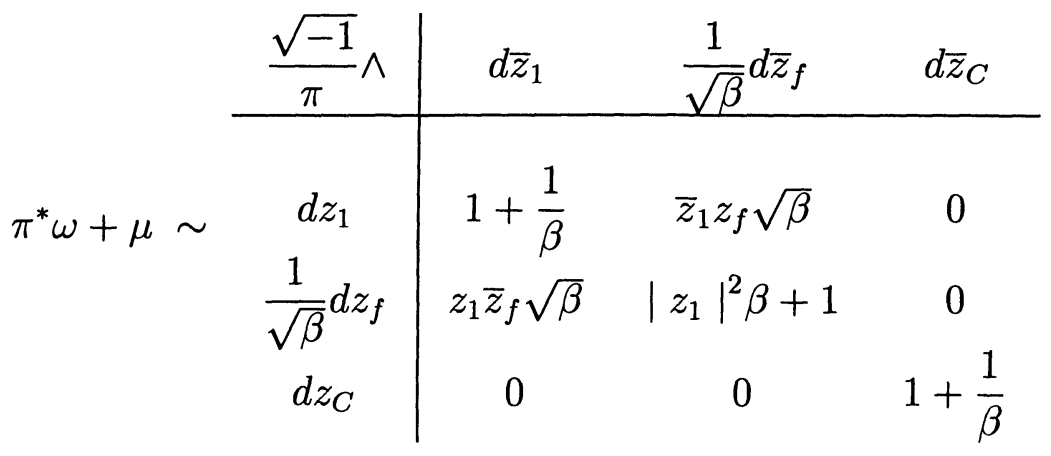

Noting that $z_{1} \log \left|z_{1}\right|^{2} \rightarrow 0$ as $z_{1} \rightarrow 0$, we see that

$\pi^{*} \omega+\mu \sim$\begin{tabular}{c|ccc}
$\frac{\sqrt{-1}}{\pi} \wedge$ & $d \bar{z}_{1}$ & $\frac{1}{\sqrt{\beta}} d \bar{z}_{f}$ & $d \bar{z}_{C}$ \\
\hline$d z_{1}$ & 1 & 0 & 0 \\
$\frac{1}{\sqrt{\beta}} d z_{f}$ & 0 & 1 & 0 \\
$d z_{C}$ & 0 & 0 & 1
\end{tabular}

i.e.

$$
\pi^{*} \omega+\mu \sim \frac{\sqrt{-1}}{\pi}\left(d z_{1} \wedge d \bar{z}_{1}+\frac{1}{\beta} d z_{f} \wedge d \bar{z}_{f}+d z_{C} \wedge d \bar{z}_{C}\right)
$$

for $z_{1}$ close enough (but not equal) to 0 . The term in the fibre direction comes from the form $\mu=-\frac{2}{\beta} c_{1}(L, h)$. Part (iii) follows directly.

We now calculate the local quasi-isometry class of $\pi^{*} \omega+\nu=$ $\pi^{*} \omega+\mu+\eta$.

LEMmA 7.3.3. Let $\tau: U \rightarrow \Delta$ be the map given by $\tau\left(z_{1}, \ldots, z_{n}\right)=$ $z_{1}$ and let $\omega_{\Delta^{*}}$ be the Poincaré form on the punctured disc. Then on $U$, for $z_{1}$ close enough (but not equal) to 0 :

$$
\pi^{*} \omega+\nu \sim \pi^{*} \omega+\mu+\tau^{*} \omega_{\Delta^{*}}
$$


Proof. Let $\omega_{E}$ be the Euclidean $(1,1)$-form $\frac{\sqrt{-1}}{\pi} \sum_{i=1}^{n} d z_{i} \wedge d \bar{z}_{i}$. From part (i) of the previous lemma, $\pi^{*} \omega+\mu \stackrel{\tilde{\geq}}{\frac{1}{\beta}} \omega_{E}$. By Proposition (5.2.4), the $(1,1)$-form $\frac{1}{\beta} \omega_{E}+\eta$ is positive and quasi-isometric to $\frac{1}{\beta} \omega_{E}+\tau^{*} \omega_{\Delta^{*}}$ for $z_{1}$ close enough (but not equal) to 0 .

This completes the proof that $l \pi^{*} \omega+\nu$ is positive for sufficiently large $l$. To obtain quasi-isometry (i) of the Proposition, we use expression (iii) of Lemma (7.3.2) and note that $\left.\beta \sim|\log | z_{1}\right|^{2} \mid$. Then we write out $\tau^{*} \omega_{\Delta^{*}}$ as

$$
\tau^{*} \omega_{\Delta^{*}}=\frac{\sqrt{-1}}{\pi}\left(\frac{d z_{1} \wedge d \bar{z}_{1}}{\left|z_{1}\right|^{2}\left(\log \left|z_{1}\right|^{2}\right)^{2}}\right)
$$

and add it to expressions (i) and (ii) of Lemma (7.3.2) to obtain the second and third quasi-isometries of the proposition.

$\S 8$. Modified Saper and Poincaré metrics for successive blow-ups and proof of Theorem I. This section contains the proof of parts (i) and (ii) of our first main result, Theorem I of $\S 2.1$. Part (iii) of Theorem I is proved in $\S 9.1$ and part (iv) in $\S 10$.

(8.1) Introduction. Let $M_{0}$ be a compact Kähler manifold with Kähler form $\omega_{0}$. Suppose that $\left\{\pi_{j}: M_{j} \rightarrow M_{j-1}\right\}$ is a finite sequence of blow-ups along smooth centres $C_{j} \subset M_{j-1}$, chosen so that $C_{j}$ has normal crossings with the total exceptional divisor $D_{j-1}$ of the composite $\pi_{1} \circ \ldots \circ \pi_{j-1}$ of the first $j-1$ blow-ups. Let $L_{j}=\left[E_{j}\right]$ be the line bundle associated with the exceptional divisor $E_{j}=\pi_{j}^{-1}\left(C_{j}\right)$. In $\S 4$ we showed that there are hermitian metrics $h_{j}$ on $L_{j}$ and positive integers $k_{j}$ such that the $(1,1)$-forms defined inductively on the compact manifolds $M_{j}$ by the equations

$$
\omega_{j}=k_{j} \pi_{j}^{*} \omega_{j-1}-c_{1}\left(L_{j}, h_{j}\right)
$$

are Kähler forms. Metrics $h_{j}$ on the line bundles $L_{j}$ induce metrics $\hat{h}_{j}$ on the line bundles $\hat{L}_{j}=\left[D_{j}\right]$, as described in $\S 3.9$. In $\S 4$ we also constructed Kähler forms $\hat{\omega}_{j}$ on $M_{j}$, using Chern forms of the line bundles $\hat{L}_{j}$ instead of $L_{j}$.

Let $s_{j}: M_{j} \rightarrow L_{j}$ be a global holomorphic section of $L_{j}$ such that $\left(s_{j}\right)=E_{j}$ and such that the norm $\left\|s_{j}\right\|$ of $s_{j}$ under the metric $h_{j}$ satisfies $\left\|s_{j}\right\|<1$ everywhere on $M_{j}$. Let $\hat{s}_{j}: M_{j} \rightarrow \hat{L}_{j}$ be the 
induced holomorphic section of $\hat{L}_{j}$ and $\left\|\hat{s}_{j}\right\|$ the norm of $\hat{s}_{j}$ under the metric $\hat{h}_{j}$. We define a Poincaré-type $(1,1)$-form $\nu_{j}$ on $M_{j}-D_{j}$ by the equation

$$
\nu_{j}=-\frac{\sqrt{-1}}{2 \pi} \partial \bar{\partial} \log \left(\log \left\|\hat{s}_{j}\right\|^{2}\right)^{2} .
$$

We will show in $\S 8$ that there are positive integers $l_{j}$ such that the $(1,1)$-forms defined inductively on the noncompact manifolds $M_{j}-D_{j}$ by the equations

$$
\omega_{S, j}=l_{j} \pi_{j}^{*} \omega_{S, j-1}+\nu_{j}
$$

are positive and consequently Kähler. The Kähler metric on $M_{j}-D_{j}$ corresponding to the form $\omega_{S, j}$ is a complete modified Saper metric, distinguished with respect to the map $\pi_{1} \circ \ldots \circ \pi_{j}: M_{j} \rightarrow M_{0}$. If the image of $D_{j}$ in $M_{0}$ consists of isolated points in $M_{0}$, this modified Saper metric is precisely a Saper distinguished metric.

It follows from Proposition (5.5.1) that the $(1,1)$-form

$$
\omega_{P, j}=\omega_{S, j}+\omega_{j}
$$

is the Kähler form of a modified Poincaré metric on $M_{j}-D_{j}$. We may replace the Kähler form $\omega_{j}$ by $\hat{\omega}_{j}$ or any other Kähler form on $M_{j}$ since all such forms are quasi-isometric.

The advantage of constructing our metrics inductively is that we can describe precisely how the metrics change with each successive blow-up. The disadvantage is that these metrics are difficult to describe totally in local coordinates, except in special cases. In $\S 9$ we will use a non-inductive method to construct Kähler homogeneous Saper metrics which can be described more precisely in local coordinates.

(8.2) Normal coordinates for successive blow-ups. In order to describe the local quasi-isometry classes of our metrics on $M_{j}$ $D_{j}$, it is useful to define normal blow-up coordinates on neighbourhoods of points in $D_{j}$.

Let $q$ be a point in $M_{j}$ at which $k$ components $\tilde{E}_{1}, \ldots, \tilde{E}_{k}$ of $D_{j}$ intersect. Each component $\tilde{E}_{i}$ is the strict transform $\tilde{E}_{j, \alpha_{i}}$ of some exceptional divisor $E_{\alpha_{i}}$. We order the components so that $\alpha_{1}<$ $\alpha_{2}<\ldots<\alpha_{k}$. Recall that local coordinates $\left(z_{1}, \ldots, z_{n}\right)$ are called 
normal for $\tilde{E}_{1}, \ldots, \tilde{E}_{k}$ if $\tilde{E}_{i}$ is given locally by the equation $z_{i}=0$ for $1 \leq i \leq k$.

If $q \notin E_{j}$ then $\pi_{j}$ is a biholomorphism on a neighbourhood of $q$. Let $\left(Z_{1}, \ldots, Z_{n}\right)$ be normal coordinates for $\pi_{j}\left(\tilde{E}_{1}\right), \ldots, \pi_{j}\left(\tilde{E}_{k}\right)$. We will say that local coordinates $\left(z_{1}, \ldots, z_{n}\right)$ near $q$ are normal blow-up coordinates for $\pi_{j}$ corresponding to $\left(Z_{1}, \ldots, Z_{n}\right)$ if $\pi_{j}$ is given locally by the equations $Z_{i}=z_{i}$ for $1 \leq i \leq n$.

If $q \in E_{j}$ then $\tilde{E}_{k}=E_{j}$ and $\pi_{j}\left(\tilde{E}_{k}\right)=C_{j}$. The remaining components $\tilde{E}_{i}=\tilde{E}_{j, \alpha_{i}}$ map to the corresponding divisors $\tilde{E}_{j-1, \alpha_{i}}$ in $M_{j-1}$. Let $\hat{E}_{i}=\tilde{E}_{j-1, \alpha_{i}}$ for $1 \leq i \leq k-1$. Since $C_{j}$ was chosen to have normal crossings with $D_{j-1}$, we may choose normal coordinates $\left(z_{1}, \ldots, z_{n}\right)$ for the divisors $\tilde{E}_{1}, \ldots, \tilde{E}_{k}$ on a neighbourhood $U$ of $q$, and normal coordinates $\left(Z_{1}, \ldots, Z_{n}\right)$ for the divisors $\hat{E}_{1}, \ldots, \hat{E}_{k-1}$ on a neighbourhood $V$ of $\pi_{j}(q)$, such that

i. $\quad C_{j}$ is given locally by the equations $Z_{\gamma}=0$ for $\gamma \in \Gamma$, where $\Gamma$ is a subset of $\{1,2, \ldots, n\}$ containing $k$ and

ii. $\pi_{j}$ is given locally by the equations

$$
\begin{array}{ll}
Z_{\gamma}=z_{k} z_{\gamma} & \text { if } \gamma \in \Gamma-\{k\} \\
Z_{i}=z_{i} & \text { if } i=k \text { or if } i \notin \Gamma .
\end{array}
$$

We will call the coordinates $\left(z_{1}, \ldots, z_{n}\right)$ normal blow-up coordinates for $\pi_{j}$ corresponding to $\left(Z_{1}, \ldots, Z_{n}\right)$.

In the case $q \in E_{j}$, there may be additional divisors $\tilde{E}_{j-1, l}$ passing through the point $\pi_{j}(q)$ whose strict transforms $\tilde{E}_{j, l}$ in $M_{j}$ do not pass through $q$. Such divisors will not be important for our local calculations near $q$. Local coordinates $W$ which are normal for the set of all divisors $\tilde{E}_{j-1, l}$ passing through a point $p \in D_{j-1}$ do not necessarily correspond to any normal blow-up coordinates $z$ in a neighbourhood of a given point $q \in \pi_{j}^{-1}(p)$.

The geometry of the map $\pi_{j}$ near $q \in E_{j}$ is easily described in normal blow-up coordinates. Local coordinates for $C_{j}$ are $Z_{C}=\left(Z_{i}\right)_{i \in \Gamma^{c}}$ where $\Gamma^{c}$ is the complement of $\Gamma$ in $\{1,2, \ldots, n\}$. Fiber coordinates of the map $E_{j} \rightarrow C_{j}$ are $\left\{z_{\gamma}\right\}_{\gamma \in \Gamma-\{k\}}$. We use the convention that the repeated index $f$ is summed over the set $\Gamma-\{k\}$ and the repeated index $C$ over the set $\Gamma^{c}$. In particular,

$$
d z_{f} \wedge d \bar{z}_{f}=\sum_{\gamma \in \Gamma-\{k\}} d z_{\gamma} \wedge d \bar{z}_{\gamma} \text { and } d z_{C} \wedge d \bar{z}_{C}=\sum_{i \in \Gamma^{c}} d z_{i} \wedge d \bar{z}_{i}
$$


(8.3) Exceptional divisors and Poincaré-type forms in normal coordinates. Before stating our main results, we will describe the divisor $D_{j}$ and the Poincaré-type form $\nu_{j}$ in normal blow-up coordinates, and compare them to the divisors and forms obtained from previous blow-ups.

For each space $M_{j}$ and for $0 \leq i \leq j-1$, let $\pi_{j, i}$ be the composite map

$$
\pi_{j, i}=\pi_{i+1} \circ \pi_{i+2} \circ \ldots \circ \pi_{j}: M_{j} \rightarrow M_{i} .
$$

Let $D_{j, i}=\pi_{j, i}^{*} D_{i}$ be the total transform of $D_{i}$ in $M_{j}$ for $1 \leq i \leq j-1$ and let $D_{j, j}=D_{j}$. Let $\nu_{j, i}=\pi_{j, i}^{*} \nu_{i}$ be the pullback of $\nu_{i}$ to $M_{j}$ for $1 \leq i \leq j-1$, and let $\nu_{j, j}=\nu_{j}$. The form $\nu_{j, i}$ is a Poincaré-type $(1,1)$-form associated with the divisor $D_{j, i}$.

Recall from $\S 3.9$ that metrics $h_{i}$ on the line bundles $L_{i}=\left[E_{i}\right]$ and sections $s_{i}$ of $L_{i}$ satisfying $\left(s_{i}\right)=E_{i}$ induce metrics $\hat{h}_{j, i}$ on $\hat{L}_{j, i}=\left[D_{j, i}\right]$ and sections $\hat{s}_{j, i}$ of $\hat{L}_{j, i}$ satisfying $\left(\hat{s}_{j, i}\right)=D_{j, i}$. We also write $\hat{s}_{j}=\hat{s}_{j, j}$ so that $\left(\hat{s}_{j}\right)=D_{j}$. Let $\left\|\hat{s}_{j, i}\right\|$ be the norm of $\hat{s}_{j, i}$ under $\hat{h}_{j, i}$. By our choice of the sections $s_{i}$, we have $\left\|\hat{s}_{j, i}\right\|<1$ on $M_{j}$. The Poincaré-type $(1,1)$-form $\nu_{j, i}$ may be written as

$$
\nu_{j, i}=-\frac{\sqrt{-1}}{2 \pi} \partial \bar{\partial} \log \left(\log \left\|\hat{s}_{j, i}\right\|^{2}\right)^{2} .
$$

We now describe the divisors $D_{j, i}$ and the corresponding Poincarétype $(1,1)$-forms $\nu_{j, i}$ near $q \in D_{j}$, using normal blow-up coordinates for $\pi_{j}$ as described in $\S 8.2$. Recall that we may write $D_{j, i}$ in terms of the irreducible divisors $\tilde{E}_{j, l}$ as

$$
D_{j, i}=\sum_{l=1}^{j} t_{i l} \tilde{E}_{j, l}
$$

where $t_{i l}$ is the multiplicity of $\tilde{E}_{j, l}$ in $D_{j, i}$. The properties of the matrix $T=\left(t_{i l}\right)=T_{j}(D, \tilde{E})$ were described in $\S 3.7$. Let $\Lambda=\left(\lambda_{i l}\right)$ be the $j \times k$ submatrix of $T$ corresponding to the irreducible divisors $\tilde{E}_{1}, \ldots, \tilde{E}_{k}$ passing through $q$. The integer $\lambda_{i l}$ is the multiplicity of $\tilde{E}_{l}$ in $D_{j, i}$. Since $T$ is nonsingular, by Proposition (3.7.4iv), the matrix $\Lambda$ has rank $k$. Let $\Lambda_{i}=\left(\lambda_{i 1}, \ldots, \lambda_{i k}\right)$ be the $i$ th row of $\Lambda$ and let

$$
z^{\Lambda_{i}}=z_{1}^{\lambda_{i 1}} z_{2}^{\lambda_{i 2}} \ldots z_{k}^{\lambda_{i k}} .
$$


The divisor $D_{j, i}$ is given locally by the equation $z^{\Lambda_{i}}=0$ and the $(1,1)$-form $\nu_{j, i}$ by

$$
\nu_{j, i}=-\frac{\sqrt{-1}}{2 \pi} \partial \bar{\partial} \log \left(\log \left(\left|z^{\Lambda_{i}}\right|^{2} g_{i}\right)\right)^{2}
$$

for some bounded positive $C^{\infty}$ function $g_{i}$.

Next we compare the divisors $D_{j, i}$ near $q$ to the divisors $D_{j-1, i}$ near $\pi_{j}(q)$ for $1 \leq i \leq j-1$. If $q \notin E_{j}$ then $\pi_{j}$ is a biholomorphism on a neighbourhood of $q$. Suppose that $q \in E_{j}$ and let $\hat{\Lambda}=\left(\hat{\lambda}_{i l}\right)$ be the $(j-1) \times(k-1)$ submatrix of the matrix $T_{j-1}(D, \tilde{E})$ corresponding to the divisors $\hat{E}_{1}, \hat{E}_{2}, \ldots, \hat{E}_{k-1}$. The strict transform of $\hat{E}_{l}$ is $\tilde{E}_{l}$ and the multiplicity of $\hat{E}_{l}$ in $D_{j-1, i}$ is $\hat{\lambda}_{i l}$. By Lemma (3.7.3), $\hat{\Lambda}$ consists of the first $j-1$ rows and $k-1$ columns of $\Lambda$, i.e. $\hat{\lambda}_{i l}=\lambda_{i l}$ for $1 \leq i \leq j-1$ and $1 \leq l \leq k-1$. Let $Z^{\hat{\Lambda}_{\imath}}=Z_{1}^{\lambda_{i 1}} Z_{2}^{\lambda_{i 2}} \ldots Z_{k-1}^{\lambda_{i, k-1}}$. The $(1,1)$-form $\nu_{j-1, i}$ is given locally by

$$
\nu_{j-1, i}=-\frac{\sqrt{-1}}{2 \pi} \partial \bar{\partial} \log \left(\log \left(\left|Z^{\hat{\Lambda}_{i}}\right|^{2} G_{i}\right)\right)^{2}
$$

for some bounded nonnegative $C^{\infty}$ function $G_{i}$. The function $G_{i}$ vanishes on those components $\tilde{E}_{j-1, l}$ of $D_{j-1, i}$ whose strict transforms $\tilde{E}_{j, l}$ do not pass through $q$. Locally near $q$ the pullback $\pi_{j}^{*} G_{i}$ is of the form $\left|z_{k}\right|^{2 \lambda} g_{i}$ for some nonnegative integer $\lambda$.

We are particularly interested in the asymptotic behaviour of the forms $\nu_{j, j}=\nu_{j}$ and $\nu_{j, j-1}=\pi_{j}^{*} \nu_{j-1}$ near $D_{j}$. We may write $\nu_{j}, \nu_{j-1}$, and $\pi_{j}^{*} \nu_{j-1}$ as $\nu_{j}=-\frac{\sqrt{-1}}{2 \pi} \partial \bar{\partial} \log \left(\beta^{2}\right), \quad \nu_{j-1}=-\frac{\sqrt{-1}}{2 \pi} \partial \bar{\partial} \log \left(B^{2}\right)$, and $\pi_{j}^{*} \nu_{j-1}=-\frac{\sqrt{-1}}{2 \pi} \partial \bar{\partial} \log \left(\beta^{\prime}\right)^{2}$ where $\beta=-\log \left\|\hat{s}_{j}\right\|^{2}$ and $B=$ $-\log \left\|\hat{s}_{j-1}\right\|^{2}$ are given in local coordinates by

$$
\beta=-\log \left(\left|z^{\Lambda_{\jmath}}\right|^{2} g_{j}\right) \quad \text { and } \quad B=-\log \left(\left|Z^{\hat{\Lambda}_{j-1}}\right|^{2} G_{j-1}\right)
$$

and $\beta^{\prime}=\pi_{j}^{*} B=\pi_{j}^{*}\left(-\log \left\|\hat{s}_{j-1}\right\|^{2}\right)=-\log \left\|\hat{s}_{j, j-1}\right\|^{2}$ by

$$
\beta^{\prime}=-\log \left(\left|z^{\Lambda_{j-1}}\right|^{2} g_{j-1}\right) \text {. }
$$

Using properties of the matrices $\Lambda$ and $\hat{\Lambda}$ derived from Lemmas (3.7.3) and (3.7.4) we obtain the following local descriptions of $\beta$ and $\beta^{\prime}$. 
LEMMA 8.3.1. If $q$ is a point in $D_{j}$ and $\left(z_{1}, \ldots, z_{n}\right)$ are normal coordinates for the irreducible components $\tilde{E}_{1}, \ldots, \tilde{E}_{k}$ of $D_{j}$ which pass through $q$, then the rate of growth of the function $\beta=-\log \left\|\hat{s}_{j}\right\|^{2}$ near $q$ is given by

i. $\beta \sim-\log \left|z_{1} z_{2} \ldots z_{k}\right|^{2}$.

If, in addition, $q \in E_{j}$, then the rate of growth of

$$
\beta^{\prime}=\pi_{j}^{*}\left(-\log \left\|\hat{s}_{j-1}\right\|^{2}\right)
$$

is given by

$$
\text { ii. } \beta^{\prime} \sim \begin{cases}-\log \left|z_{1} z_{2} \ldots z_{k}\right|^{2} & \text { if } C_{j} \subset D_{j-1} . \\ -\log \left|z_{1} z_{2} \ldots z_{k-1}\right|^{2} & \text { if } C_{j} \not \subset D_{j-1} \text { and } k \geq 2 . \\ 1 & \text { if } C_{j} \not \subset D_{j-1} \text { and } k=1 .\end{cases}
$$

Proof. Recall that the entries of $\Lambda_{j}$ are all positive, i.e. the multiplicity $\lambda_{j, i}$ of $\tilde{E}_{i}$ in $D_{j}$ is positive for all $i$, by Proposition (3.7.4 i). This gives us quasi-isometry (i).

Now assume that $q \in E_{j}$. The first $k-1$ entries of $\Lambda_{j}, \hat{\Lambda}_{j-1}$, and $\Lambda_{j-1}$ are identical (Lemma (3.7.3 iii)) since these entries are the multiplicities of the strict transforms of $E_{\alpha_{1}}, E_{\alpha_{2}}, \ldots, E_{\alpha_{k-1}}$ in the divisors $D_{j}, D_{j-1}$, and $D_{j, j-1}=\pi_{j}^{*} D_{j-1}$. The $k$ th entries of $\Lambda_{j}$ and $\Lambda_{j-1}$ are related by the equation $\lambda_{j, k}=\lambda_{j-1, k}+1$ (Lemma (3.7.3 iv)) since $D_{j}=D_{j, j-1}+E_{j}$. The entry $\lambda_{j-1, k}$ is positive if and only if $C_{j} \subset D_{j-1}$ by Proposition $(3.7 .4 \mathrm{v})$. This gives us quasi-isometry (ii).

In $\S 5$ we compared Poincaré-type forms $\nu$ to pullbacks of the Poincaré form $\omega_{\Delta^{*}}$ by suitable monomial maps. For each $i$ such that $\Lambda_{i}$ has at least one positive entry, let $\tau_{i}: U \rightarrow \Delta$ be the monomial map given by

$$
\tau_{i}\left(z_{1}, z_{2}, \ldots, z_{n}\right)=z^{\Lambda_{i}}
$$

If $\Lambda_{i}=0$, let $\tau_{i}$ be a constant map to a point in $\Delta^{*}$ so that $\tau_{i}^{*} \omega_{\Delta^{*}}=0$.

(8.4) Main results. We can now generalize the results of $\S 7$ to successive blow-ups. Our first theorem describes how complete Kähler modified Saper metrics may be constructed inductively on the noncompact spaces $M_{j}-D_{j}$ by adding a Poincaré-type form on $M_{j}-D_{j}$ to a large enough multiple of the pullback of the Kähler form of the 
previous metric. The proof of the following theorem is to be found in $\S \S 8.5$ and 8.6 .

THEOREM 8.4.1. (Complete Kähler modified Saper metrics for successive blow-ups.) There exist positive integers $l_{j}$ such that the $(1,1)$-forms defined inductively on the manifolds $M_{j}-D_{j}$ by the equations

$$
\omega_{S, 0}=\omega_{0} \text { and } \omega_{S, j}=l_{j} \pi_{j}^{*} \omega_{S, j-1}+\nu_{j} \quad \text { for } j \geq 1
$$

are Kähler forms. The Kähler metric on $M_{j}-D_{j}$ associated with $\omega_{S, j}$ is a complete modified Saper metric which is distinguished with respect to the map $\pi_{j, 0}=\pi_{1} \circ \pi_{2} \circ \ldots \circ \pi_{j}$.

The quasi-isometry class of $\omega_{S, j}$ may be described as follows: If $q \notin E_{j}$, then on a neighbourhood $U$ of $q, \omega_{S, j} \sim \pi_{j}^{*} \omega_{S, j-1}$.

If $q \in E_{j}$, if $k$ is the number of components of $D_{j}$ intersecting at $q$, and if $\left(z_{1}, \ldots, z_{n}\right)$ are normal blow-up coordinates for $\pi_{j}$ on a neighbourhood $U$ of $q$, then locally

(i) $\omega_{S, j} \sim \pi_{j}^{*} \omega_{S, j-1}$

$$
+\frac{\sqrt{-1}}{\pi}\left(\frac{1}{\left.|\log | z_{1} z_{2} \ldots z_{k}\right|^{2} \mid} d z_{f} \wedge d \bar{z}_{f}\right)+\tau_{j}^{*} \omega_{\Delta^{*}}, \text { and }
$$

(ii) $\omega_{S, j} \stackrel{\sim}{\geq} \pi_{j, 0}^{*} \omega_{0}+\frac{\sqrt{-1}}{\pi}\left(\frac{1}{\left(\log \left|z_{1} z_{2} \ldots z_{k}\right|^{2}\right)^{2}} \sum_{i=1}^{k} \frac{d z_{i} \wedge d \bar{z}_{i}}{\left|z_{i}\right|^{2}}\right.$

$$
\left.+\frac{1}{\left.|\log | z_{1} z_{2} \ldots z_{k}\right|^{2} \mid} \sum_{i=1}^{n} d z_{i} \wedge d \bar{z}_{i}\right) \text {. }
$$

Note that completeness follows directly from quasi-isometry (i) by Proposition (6.1.1), since the monomial map $\tau_{j}$ is given by

$$
\tau_{j}\left(z_{1}, \ldots, z_{n}\right)=z^{\Lambda_{j}}=z_{1}^{\lambda_{j 1}} z_{2}^{\lambda_{j 2}} \ldots z_{k}^{\lambda_{j k}}
$$

and all the integers $\lambda_{j 1}, \ldots, \lambda_{j k}$ are positive.

Quasi-isometry (i) means that locally near the exceptional divisor $E_{j}$ of $\pi_{j}$, the $j$ th modified Saper metric looks like the pullback of the $(j-1)$ st metric plus a small term in the fibre directions plus a term with Poincaré-type growth in the variables $z_{1}, \ldots, z_{k}$. Inequality (ii) 
means that the modified Saper metric on $M_{j}-D_{j}$ is locally bounded below by the sum of the incomplete metric induced from $M_{0}$ and a Saper distinguished metric on $M_{j}-D_{j}$.

We noted in Proposition (5.5.1) that the sum of a modified Saper metric on $M_{j}-D_{j}$ and the restriction to $M_{j}$ of a Kähler metric on $M_{j}$ is a modified Poincaré metric. The following theorem also describes the relationship between our modified Poincaré metrics on $M_{j}-D_{j}$ and $M_{j-1}-D_{j-1}$. The proof is in $\S 8.7$.

TheOREM 8.4.2. (Complete Kähler modified Poincaré metrics for successive blow-ups.) The Kähler forms

$$
\omega_{P, j}=\omega_{S, j}+\omega_{j}
$$

determine complete modified Poincaré metrics on the manifolds $M_{j}-$ $D_{j}$.

The metric associated with $\omega_{P, 1}$ is a true Poincaré metric. For $j \geq 2$ the quasi-isometry class of $\omega_{P, j}$ may be described as follows: If $q \notin E_{j}$, then on a neighbourhood $U$ of $q, \omega_{P, j} \sim \pi_{j}^{*} \omega_{P, j-1}$. If $q \in E_{j}$ and if $\left(z_{1}, \ldots, z_{n}\right)$ are normal blow-up coordinates for $\pi_{j}$ on a neighbourhood $U$ of $q$, then locally

$$
\begin{aligned}
& \omega_{P, j} \sim \pi_{j}^{*} \omega_{P, j-1}+\frac{\sqrt{-1}}{\pi} d z_{f} \wedge d \bar{z}_{f}+\tau_{j}^{*} \omega_{\Delta^{*}}, \text { and } \\
& \omega_{P, j} \sim \sum_{i=1}^{j} \tau_{i}^{*} \omega_{\Delta^{*}}+\frac{\sqrt{-1}}{\pi} \sum_{i=1}^{n} d z_{i} \wedge d \bar{z}_{i} .
\end{aligned}
$$

Notice that the modified Saper and Poincaré metrics differ only in the order of growth of the fiber terms $d z_{f} \wedge d \bar{z}_{f}$. These terms correspond to multiples of a Chern form of the line bundle $\hat{L}_{j}=\left[D_{j}\right]$. Quasi-isometry (ii) simply states that $\omega_{P, j}$ is a modified Poincaré metric in the sense of (1.6.1).

Let $\omega_{\text {Sap }}$ and $\omega_{\text {Poinc }}$ be the fundamental forms, respectively, of any Saper distinguished metric and any Poincaré metric on $M_{j}-D_{j}$, neither necessarily Kähler. Using the local descriptions of $\omega_{S, j}$ and $\omega_{P, j}$, we obtain

THEOREM 8.4.3. (Global bounds for modified Saper and Poincaré metrics.) The modified Saper metric on $M_{j}-D_{j}$ associated with 
$\omega_{S, j}$ is bounded below by the sum of the metric induced from $M_{0}$ and a Saper distinguished metric, and is bounded above by the modified Poincaré metric associated with $\omega_{P, j}$. This modified Poincaré metric is bounded above by a true Poincaré metric. More concisely:

$$
\pi_{j, 0}^{*} \omega_{0}+\omega_{\text {Sap }} \stackrel{\sim}{\leq} \omega_{S, j}<\omega_{P, j} \stackrel{\sim}{\leq} \omega_{\text {Poinc }}
$$

Proof. The first inequality follows directly from part (ii) of Theorem (8.4.1) and the second from the definition of $\omega_{P, j}$. The local quasi-isometry class of the form $\omega_{\text {Poinc }}$ is given by

$$
\omega_{\text {Poinc }} \sim \frac{\sqrt{-1}}{\pi}\left(\sum_{i=1}^{k} \frac{d z_{i} \wedge d \bar{z}_{i}}{\left|z_{i}\right|^{2}\left(\log \left|z_{i}\right|^{2}\right)^{2}}+\sum_{i=1}^{n} d z_{i} \wedge d \bar{z}_{i}\right)
$$

which dominates $\omega_{P, j}$, by Lemma (5.3.6).

When more is known about the relationships between the centres $C_{i}$ and the exceptional divisors $D_{i-1}$, we can be more precise in our descriptions of the metrics. Let $\omega_{P, \text { hom }}$ be the fundamental form of a modified Poincaré metric on $M_{j}-D_{j}$ which is homogeneous in the sense of (1.6.2). The following theorem is proved in $\S 8.8$. We allow for the possibility that $D_{j}$ may have several connected components, but we need only prove the theorem for the case of a single connected component, since each blow-up map $\pi_{i}$ is a biholomorphism away from its own exceptional divisor $E_{i}$.

THEOREM 8.4.4. (Metrics for the case $C_{i} \subset D_{i-1}$.) Suppose that for $2 \leq i \leq j$, the centre $C_{i}$ for the ith blow-up is either contained in the total exceptional divisor $D_{i-1}$ of the first $i-1$ blow-ups or is disjoint from $D_{i-1}$. Then the Kähler metric associated with $\omega_{S, j}$ is a homogeneous Saper metric in the sense of (1.8.4) and the Kähler metric associated with $\omega_{P, j}$ is a homogeneous Poincaré metric. More concisely:

$$
\omega_{S, j} \sim \pi_{j, 0}^{*} \omega_{0}+\omega_{\text {Sap }} \text { and } \omega_{P, \jmath} \sim \omega_{P, \mathrm{hom}}
$$

If, in addition, $\operatorname{dim} C_{1}=0$ and $\operatorname{dim} C_{i}=0$ for each $i$ such that $C_{i}$ is disjoint from $D_{i-1}$ (i.e. the image of $D_{j}$ in $M_{0}$ consists of 
isolated points in $M_{0}$ ), then the modified Saper metric is exactly a Saper distinguished metric, i.e. $\omega_{S, j} \sim \omega_{\text {Sap }}$.

Outline of proofs. We proved Theorems (8.4.1) - (8.4.4) for a single blow-up in $\S 7$. In that case, $\omega_{S, 1} \sim \pi_{1}^{*} \omega_{0}+\omega_{\text {Sap }}$ and $\omega_{P, 1} \sim \omega_{\text {Poinc }} \sim$ $\omega_{P, \text { hom }}$.

Now fix $m \geq 2$ and assume that Theorems (8.4.1) - (8.4.4) are true for $1 \leq j \leq m-1$. For the rest of $\S 8$ we will let $\pi=\pi_{m}$. Consider the forms

$$
\omega_{S, m}=l_{m} \pi^{*} \omega_{S, m-1}+\nu_{m} \quad \text { and } \quad \omega_{P, m}=\omega_{S, m}+\omega_{m} .
$$

The form $\nu_{m}$ is hermitian and $d$-closed so to show that $\omega_{S, m}$ and $\omega_{P, m}$ are Kähler, we need only show that $\omega_{S, m}$ is positive on $M_{m}-D_{m}$.

The $(1,1)$-form $\nu=\nu_{m}$ may be written as $\nu=-\frac{\sqrt{-1}}{2 \pi} \partial \bar{\partial} \log \left(\beta^{2}\right)$ where $\beta=-\log \left\|\hat{s}_{m}\right\|^{2}$ and $\left\|\hat{s}_{m}\right\|$ is the norm of the section $\hat{s}_{m}: M_{m} \rightarrow \hat{L}_{m}$ described in $\S 8.1$. As in $\S 5.1$ we decompose $\nu$ as $\nu=\mu+\eta$ where

$$
\mu=-\frac{\sqrt{-1}}{\pi} \frac{\partial \bar{\partial} \beta}{\beta}=-\frac{2}{\beta} c_{1}\left(\left[D_{m}\right], \hat{h}_{m}\right) \text { and } \eta=\frac{\sqrt{-1}}{\pi} \frac{\partial \beta \wedge \bar{\partial} \beta}{\beta^{2}} .
$$

By Lemma (8.3.1), the rate of growth of $\beta$ near $q \in D_{m}$ is given locally in normal coordinates by $\beta \sim-\log \left|z_{1} z_{2} \ldots z_{k}\right|^{2}$.

Application of our inductive assumption of Theorem (8.4.1) for $j=m-1$ is complicated by having to deal with two sets of local coordinates near $\pi(q)$ in $M_{m-1}$ : coordinates $W$ which are normal for the collection of all irreducible components $\hat{E}_{1}, \ldots, \hat{E}_{K}$ of $D_{m-1}$ passing through $\pi(q)$, and coordinates $Z$, as described in $\S 8.2$, which are normal for $C_{m}$ and those irreducible components $\hat{E}_{i}$ of $D_{m-1}$ whose strict transforms in $M_{m}$ pass through $q$. Our local coordinates $z$ on $M_{m}$ are normal blow-up coordinates corresponding to $Z$. To use our inductive assumption, written in $W$ coordinates, we first convert to $Z$ or $z$ coordinates.

To simplify notation we will let

$$
\phi=\frac{\sqrt{-1}}{\pi} \sum_{i=1}^{k} \frac{d z_{i} \wedge d \bar{z}_{i}}{\left|z_{i}\right|^{2}} \text { and } \Phi=\frac{\sqrt{-1}}{\pi} \sum_{i=1}^{K} \frac{d W_{i} \wedge d \bar{W}_{i}}{\left|W_{i}\right|^{2}}
$$


Throughout $\S 8$ we will use freely the quasi-isometries

$$
\omega_{m} \sim \frac{\sqrt{-1}}{\pi} \sum_{i=1}^{n} d z_{i} \wedge d \bar{z}_{i} \text { and } \omega_{m-1} \sim \frac{\sqrt{-1}}{\pi} \sum_{i=1}^{n} d W_{i} \wedge d \bar{W}_{i}
$$

where $\omega_{m}$ and $\omega_{m-1}$ may be replaced by the positive $(1,1)$-forms corresponding to any hermitian metrics on $M_{m}$ and $M_{m-1}$ respectively.

It is convenient to write our inductive assumption in terms of the function $B=-\log \left\|\hat{s}_{m-1}\right\|^{2}$. By Lemma (8.3.1) with $j=m-1$, we have

$$
B \sim-\log \left|W_{1} W_{2} \ldots W_{K}\right|^{2}
$$

near $\pi(q)$. Our inductive assumption of (8.4.1ii) for $j=m-1$ may be written as

$$
\omega_{S, m-1} \stackrel{\sim}{\geq} \pi_{m-1,0}^{*} \omega_{0}+\frac{1}{B^{2}} \Phi+\frac{1}{B} \omega_{m-1}
$$

from which it follows that $\omega_{S, m-1} \stackrel{\sim}{\geq} \frac{1}{B} \omega_{m-1}$ and

$$
\pi^{*} \omega_{S, m-1} \stackrel{\sim}{\geq} \frac{1}{\beta^{\prime}} \pi^{*} \omega_{m-1}
$$

where $\beta^{\prime}=\pi^{*} B$. Lemma (8.3.1) gives us a description in $z$ coordinates of the rate of growth of $\beta^{\prime}$ near $q$.

The proof of positivity and the calculations of the quasi-isometry classes of Theorem (8.4.1) are done for $q \notin E_{m}$ in $\S 8.5$ and for $q \in E_{m}$ in $\S 8.6$. We finish the proof of Theorem (8.4.2) in $\S 8.7$ and Theorem (8.4.4) in $§ 8.8$.

\section{(8.5) Quasi-isometry class of $\omega_{S, m}$ near $q \notin E_{m}$.}

Proof of Theorem (8.4.1) for $q \notin E_{m}$. If $q$ is not in the exceptional divisor $E_{m}=\pi^{-1}\left(C_{m}\right)$ it is easy to show that the form $\omega_{S, m}=$ $l \pi^{*} \omega_{S, m-1}+\nu$ is locally quasi-isometric to $\pi^{*} \omega_{S, m-1}$ for $l \gg 0$. We need only show that $l \pi^{*} \omega_{S, m-1}$ dominates $\nu=\mu+\eta$ locally. If $q \notin$ $D_{m}$ then $\pi^{*} \omega_{S, m-1}$ is positive and $\nu$ is bounded on a neighbourhood of $q$ and we are done. 
Suppose that $q \in D_{m}$ but $q \notin E_{m}$. The map $\pi$ is a biholomorphism near $q$ so $\pi^{*} \omega_{S, m-1}$ has the same description in local coordinates as $\omega_{S, m-1}$. By our inductive assumption that $\omega_{S, m-1}$ satisfies quasi-isometry (ii) of Theorem (8.4.1), we have

$$
\pi^{*} \omega_{S, m-1} \simeq \frac{1}{\beta^{2}} \phi+\frac{1}{\beta} \omega_{m}
$$

where $\phi$ is as in equation (8.4.6). Recall that $\mu=-\frac{2}{\beta} c_{1}\left(\left[D_{m}\right], \hat{h}_{m}\right)$. Then $\mu$ is locally dominated by $l \frac{1}{\beta} \omega_{m}$ and hence by $l \pi^{*} \omega_{S, m-1}$ for $l$ large enough. To see that $\eta$ is also dominated by $l \pi^{*} \omega_{S, m-1}$, we use Proposition (5.2.4), which tells us that locally $\frac{1}{\beta} \omega_{m}+\eta \sim \frac{1}{\beta} \omega_{m}+$ $\tau_{m}^{*} \omega_{\Delta^{*}}$ where $\tau_{m}\left(z_{1}, \ldots, z_{n}\right)=z^{\Lambda_{m}}$. The form $\phi_{m}=\beta^{2} \tau_{m}^{*} \omega_{\Delta^{*}}$ is positive semi-definite, and by Lemma (5.3.2), $\phi \tilde{\geq} \phi_{m}$. Then $\frac{1}{\beta^{2}} \phi \simeq$ $\tau_{m}^{*} \omega_{\Delta^{*}}$ and $\pi^{*} \omega_{S, m-1} \tilde{\geq} \tau_{m}^{*} \omega_{\Delta^{*}}+\frac{1}{\beta} \omega_{m} \sim \eta+\frac{1}{\beta} \omega_{m} \stackrel{\tilde{\geq}}{\eta}$.

(8.6) Quasi-isometry class of $\omega_{S, m}$ near $q \in E_{m}$.

Proof of Theorem (8.4.1) for $q \in E_{m}$. Recall that

$$
\omega_{S, m}=l \pi^{*} \omega_{S, m-1}+\nu
$$

where $\nu=-\frac{\sqrt{-1}}{2 \pi} \partial \bar{\partial} \log \left(\beta^{2}\right)$ has the decomposition $\nu=\mu+\eta$ described in (8.4.5) and $\beta \sim-\log \left|z_{1} z_{2} \ldots z_{k}\right|^{2}$ near $q$. Our inductive assumption gives us estimate (8.4.9): $\pi^{*} \omega_{S, m-1} \stackrel{\sim}{\simeq} \frac{1}{\beta^{\prime}} \pi^{*} \omega_{m-1}$. By Lemma (8.3.1), $\beta^{\prime} \tilde{\leq} \beta$. Hence

$$
\pi^{*} \omega_{S, m-1} \simeq \frac{1}{\beta} \pi^{*} \omega_{m-1} .
$$

We will first show that $l \frac{1}{\beta} \pi^{*} \omega_{m-1}+\mu$ and $l \frac{1}{\beta} \pi^{*} \omega_{m-1}+\nu$ are positive on $M_{m}-D_{m}$ for $l \gg 0$. We will then calculate the quasi-isometry class of $l \frac{1}{\beta^{\prime}} \pi^{*} \omega_{m-1}+\mu$ in local coordinates to obtain part (i) of Theorem (8.4.1). To prove part (ii), we estimate the size of the terms in $\omega_{S, m}$ which have Poincare-type growth, using properties of the multiplicity matrix $T_{m}(D, \tilde{E})$ from $\S 3$ and results on Poincarétype forms from $\S 5$. 
LEMMA 8.6.2. For $l \gg 0$ the $(1,1)$-form $l \frac{1}{\beta} \pi^{*} \omega_{m-1}+\mu$ is positive on $M_{m}-D_{m}$ and

$$
l \frac{1}{\beta} \pi^{*} \omega_{m-1}+\mu \sim \frac{1}{\beta} \omega_{m}
$$

Proof. From (8.4.5) we have $\mu=-\frac{2}{\beta} c_{1}\left(\left[D_{m}\right], \hat{h}_{m}\right)$. Recall from Corollary (4.3.2) that there is a Kähler form $\hat{\omega}_{m-1}$ on $M_{m-1}$ such that

$$
l \pi^{*} \hat{\omega}_{m-1}-c_{1}\left(\left[D_{m}\right], \hat{h}_{m}\right)>0
$$

on $M_{m}$ for $l \gg 0$. Then $l \pi^{*} \hat{\omega}_{m-1}+\beta \mu>0$ for $l \gg 0$. Since all metrics on a compact manifold are quasi-isometric, we have

$$
l \frac{1}{\beta} \pi^{*} \omega_{m-1}+\mu \sim \frac{1}{\beta} \omega_{m}
$$

for the positive $(1,1)$-forms $\omega_{m-1}$ and $\omega_{m}$ of any hermitian metrics on $M_{m-1}$ and $M_{m}$ respectively.

Since $\beta^{\prime} \stackrel{\sim}{\leq} \beta$ we have

Corollary 8.6.3. For $l \gg 0$

$$
l \frac{1}{\beta^{\prime}} \pi^{*} \omega_{m-1}+\mu \sim \frac{1}{\beta^{\prime}} \pi^{*} \omega_{m-1}+\frac{1}{\beta} \omega_{m}
$$

The next lemma is a consequence of Proposition (5.2.4).

LEMma 8.6.4. For $z_{1}, \ldots, z_{k}$ close enough (but not equal) to 0 , the $(1,1)$-form $\frac{1}{\beta} \omega_{m}+\eta$ is positive and

$$
\frac{1}{\beta} \omega_{m}+\eta \sim \frac{1}{\beta} \omega_{m}+\tau_{m}^{*} \omega_{\Delta^{*}}
$$

Combining Lemmas (8.6.2) and (8.6.4) we obtain the following quasi-isometry which, together with inequality (8.6.1), implies that $\omega_{S, m}$ is positive.

COROLlaRY 8.6.5. For $z_{1}, \ldots, z_{k}$ close enough (but not equal) to 0 and $l \gg 0$

$$
l \frac{1}{\beta} \pi^{*} \omega_{m-1}+\nu \sim \frac{1}{\beta} \omega_{m}+\tau_{m}^{*} \omega_{\Delta^{*}}
$$


Using the inequality $\beta^{\prime} \stackrel{\sim}{\leq} \beta$ again gives us a variation of Corollary (8.6.5) which is used in the conclusion of the proof of Theorem (8.4.1i) below.

Corollary 8.6.6. For $z_{1}, \ldots, z_{k}$ close enough (but not equal) to 0 and $l \gg 0$

$$
l \frac{1}{\beta^{\prime}} \pi^{*} \omega_{m-1}+\nu \sim \frac{1}{\beta^{\prime}} \pi^{*} \omega_{m-1}+\frac{1}{\beta} \omega_{m}+\tau_{m}^{*} \omega_{\Delta^{*}}
$$

Next we describe the $(1,1)$-form of Corollary (8.6.3) in local coordinates. We show that $\frac{1}{\beta^{\prime}} \pi^{*} \omega_{m-1}$ dominates all terms in $\frac{1}{\beta} \omega_{m}$ except possibly those in the direction of fibres of the map $E_{m} \rightarrow C_{m}$.

Lemma 8.6.7. For $z_{1}, \ldots, z_{k}$ close enough (but not equal) to 0

$$
\begin{aligned}
& \frac{1}{\beta^{\prime}} \pi^{*} \omega_{m-1}+\frac{1}{\beta} \omega_{m} \sim \frac{\sqrt{-1}}{\pi}\left(\frac{1}{\beta^{\prime}}\left(d z_{k} \wedge d \bar{z}_{k}+d z_{C} \wedge d \bar{z}_{C}\right)\right. \\
&\left.+\frac{1}{\beta} d z_{f} \wedge d \bar{z}_{f}\right) \\
& \sim \frac{1}{\beta^{\prime}} \pi^{*} \omega_{m-1}+\frac{\sqrt{-1}}{\pi} \frac{1}{\beta} d z_{f} \wedge d \bar{z}_{f}
\end{aligned}
$$

Proof. We write out $\pi^{*} \omega_{m-1}$ in local coordinates, using the notation of $\S 8.2$ :

$$
\begin{aligned}
\pi^{*} \omega_{m-1} & \sim \pi^{*}\left(\frac{\sqrt{-1}}{\pi} \sum_{i=1}^{n} d Z_{i} \wedge d \bar{Z}_{i}\right) \\
= & \frac{\sqrt{-1}}{\pi}\left(d z_{k} \wedge d \bar{z}_{k}+d z_{C} \wedge d \bar{z}_{C}\right. \\
\quad & \left.\quad+\left(z_{f} d z_{k}+z_{k} d z_{f}\right) \wedge\left(\bar{z}_{f} d \bar{z}_{k}+\bar{z}_{k} d \bar{z}_{f}\right)\right)
\end{aligned}
$$


where the repeated index $f$ indicates summation over the set $\Gamma-\{k\}$. Adding $\frac{1}{\beta} \omega_{m}$ and writing the forms in chart format we have:

$$
\begin{aligned}
& \frac{1}{\beta^{\prime}} \pi^{*} \omega_{m-1}+\frac{1}{\beta} \omega_{m} \\
& \begin{array}{c|ccc}
\frac{\sqrt{-1}}{\pi} \wedge & d \bar{z}_{k} & \mathrm{~d} \bar{z}_{f} & d \bar{z}_{C} \\
\hline d z_{k} & \frac{1}{\beta^{\prime}}+\frac{1}{\beta} & \bar{z}_{k} z_{f} \frac{1}{\beta^{\prime}} & 0 \\
d z_{f} & z_{k} \bar{z}_{f} \frac{1}{\beta^{\prime}} & \left|z_{k}\right|^{2} \frac{1}{\beta^{\prime}}+\frac{1}{\beta} & 0 \\
d z_{C} & 0 & 0 & \frac{1}{\beta^{\prime}}+\frac{1}{\beta}
\end{array} \\
& \begin{array}{c|ccc}
\frac{\sqrt{-1}}{\pi} \wedge & \frac{1}{\sqrt{\beta^{\prime}}} d \bar{z}_{k} & \frac{1}{\sqrt{\beta}} d \bar{z}_{f} & \frac{1}{\sqrt{\beta^{\prime}}} d \bar{z}_{C} \\
\sim & 1+\frac{\beta^{\prime}}{\beta} & \bar{z}_{k} z_{f} \sqrt{\frac{\beta}{\beta^{\prime}}} & 0 \\
& \begin{array}{c}
\frac{1}{\sqrt{\beta^{\prime}}} d z_{k} \\
\frac{1}{\sqrt{\beta}} d z_{f}
\end{array} z_{k} \bar{z}_{f} \sqrt{\frac{\beta}{\beta^{\prime}}} & \left|z_{k}\right|^{2} \frac{\beta}{\beta^{\prime}}+1 & 0 \\
\frac{1}{\sqrt{\beta^{\prime}}} d z_{C} & 0 & 0 & 1+\frac{\beta^{\prime}}{\beta}
\end{array}
\end{aligned}
$$

From Lemma (8.3.1), $\beta \sim \beta^{\prime}$ if $C_{m} \subset D_{m-1}$ and $1 \stackrel{\sim}{\leq} \frac{\beta}{\beta^{\prime}} \stackrel{\sim}{\leq}-\log \left|z_{k}\right|^{2}$ for $z_{1}, \ldots, z_{k}$ near 0 if $C_{m} \not \subset D_{m-1}$. In both cases, $z_{k} \frac{\beta}{\beta^{\prime}} \rightarrow 0$ and $\frac{\beta^{\prime}}{\beta}$ is bounded as $z_{k} \rightarrow 0$. This proves quasi-isometry (i). Quasi-isometry (ii) follows because we have showed that the form $\frac{1}{\beta^{\prime}} \pi^{*} \omega_{m-1}$ dominates all terms in $\frac{1}{\beta} \omega_{m}$ except $\frac{\sqrt{-1}}{\pi} \frac{1}{\beta} d z_{f} \wedge d \bar{z}_{f}$.

Conclusion of the proof of Theorem (8.4.1 i). From Corollary (8.6.6) and Lemma (8.6.7) we obtain the quasi-isometry

$$
l \frac{1}{\beta^{\prime}} \pi^{*} \omega_{m-1}+\nu \sim \frac{1}{\beta^{\prime}} \pi^{*} \omega_{m-1}+\frac{\sqrt{-1}}{\pi} \frac{1}{\beta} d z_{f} \wedge d \bar{z}_{f}+\tau_{m}^{*} \omega_{\Delta^{*}}
$$

for $l \gg 0$ and $z_{1}, \ldots, z_{k}$ close enough (but not equal) to 0 . Applying inequality (8.4.9), which says that $\pi^{*} \omega_{S, m-1} \underset{\geq}{\simeq} \frac{1}{\beta^{\prime}} \pi^{*} \omega_{m-1}$, we obtain 
quasi-isometry (i) of Theorem (8.4.1):

$$
\omega_{S, m}=l \pi^{*} \omega_{S, m-1}+\nu \sim \pi^{*} \omega_{S, m-1}+\frac{\sqrt{-1}}{\pi} \frac{1}{\beta} d z_{f} \wedge d \bar{z}_{f}+\tau_{m}^{*} \omega_{\Delta^{*}} .
$$

Conclusion of the proof of Theorem (8.4.1 ii). We start with the pullback of the forms of our inductive assumption (8.4.8):

$$
\pi^{*} \omega_{S, m-1} \stackrel{\sim}{\geq} \pi_{m, 0}^{*} \omega_{0}+\pi^{*}\left(\frac{1}{B^{2}} \Phi+\frac{1}{B} \omega_{m-1}\right)
$$

where $\Phi$ is as in (8.4.6). Notice that if $\omega_{\text {Sap }, m-1}$ is the fundamental form of a distinguished Saper metric on $M_{m-1}-D_{m-1}$ then

$$
\omega_{\mathrm{Sap}, m-1} \sim \frac{1}{B^{2}} \Phi+\frac{1}{B} \omega_{m-1}
$$

near $\pi(q)$. Applying Corollary (8.6.5) and the inequality $\pi^{*} B=$ $\beta^{\prime} \stackrel{\sim}{\leq} \beta$ gives

$$
l \pi^{*} \omega_{S, m-1}+\nu \stackrel{\sim}{\geq} \pi_{m, 0}^{*} \omega_{0}+\pi^{*} \omega_{\text {Sap }, m-1}+\frac{1}{\beta} \omega_{m}+\tau_{m}^{*} \omega_{\Delta^{*}}
$$

Part (ii) of Theorem (8.4.1) can be written as

$$
l \pi^{*} \omega_{S, m-1}+\nu \stackrel{\sim}{\geq} \pi_{m, 0}^{*} \omega_{0}+\frac{1}{\beta^{2}} \phi+\frac{1}{\beta} \omega_{m}
$$

with $\phi$ as in (8.4.6), and follows from the next lemma.

LEMMA 8.6.9. For $z_{1}, \ldots, z_{k}$ close enough (but not equal to) 0 ,

$$
\pi^{*} \omega_{\mathrm{Sap}, m-1}+\frac{1}{\beta} \omega_{m}+\tau_{m}^{*} \omega_{\Delta^{*}} \geq \frac{1}{\beta^{2}} \phi+\frac{1}{\beta} \omega_{m}
$$

Proof. The quasi-isometry class of $\omega_{\text {Sap }, m-1}$ near $\pi(q)$ was described in terms of local coordinates $W$ which are normal for all irreducible components $\hat{E}_{1}, \ldots, \hat{E}_{K}$ of $D_{m-1}$ passing through $\pi(q)$. We will first rewrite some of the terms of $\omega_{\mathrm{Sap}, m-1}$ in the $Z$ coordinates of $\S 8.2$, which are normal for $C_{m}$ and those components 
$\hat{E}_{1}, \ldots, \hat{E}_{k-1}$ of $D_{m-1}$ whose strict transforms in $M_{m}$ pass through $q$. We will then use our $\S 3$ description of the relationship between the multiplicities of the components of $D_{m-1}$ and $D_{m}$ and our $\S 5$ results on Poincaré-type forms.

Recall (Corollary (5.3.5)) that the quasi-isometry class of a distinguished Saper metric looks the same in any system of normal coordinates $W$ for $\hat{E}_{1}, \ldots, \hat{E}_{K}$. We may choose $W_{i}=Z_{i}$ for $1 \leq i \leq k-1$ and then augment the collection $W_{1}, \ldots, W_{k-1}$ to a collection of normal coordinates $W_{1}, \ldots, W_{n}$ for $\hat{E}_{1}, \ldots, \hat{E}_{K}$. Then

$$
\omega_{\text {Sap }, m-1} \sim \frac{1}{B^{2}} \Phi+\frac{1}{B} \omega_{m-1} \geq \frac{\sqrt{-1}}{\pi} \frac{1}{B^{2}} \sum_{i=1}^{k-1} \frac{d Z_{i} \wedge d \bar{Z}_{i}}{\left|Z_{i}\right|^{2}}+\frac{1}{B} \omega_{m-1} .
$$

Let

$$
\phi_{i}=\pi^{*}\left(\frac{\sqrt{-1}}{\pi} \frac{d Z_{i} \wedge d \bar{Z}_{i}}{\left|Z_{i}\right|^{2}}\right) .
$$

Using $\pi^{*} B=\beta^{\prime} \stackrel{\sim}{\leq} \beta$, we obtain

$$
\pi^{*} \omega_{\text {Sap }, m-1} \stackrel{\sim}{\geq} \frac{1}{\beta^{2}} \sum_{i=1}^{k-1} \phi_{i}+\frac{1}{\beta} \pi^{*} \omega_{m-1} .
$$

The forms $\phi_{i}$ have a simple description in $z$ coordinates, since for $1 \leq i \leq k-1$ the pullback of $Z_{i}$ under $\pi$ is

$$
\pi^{*} Z_{i}=z_{i} z_{k}^{\delta_{i}}
$$

where $\delta_{i}=1$ if $C_{m} \subset \hat{E}_{i}$ and $\delta_{i}=0$ otherwise.

Recall that $\tau_{m}\left(z_{1}, \ldots, z_{n}\right)=z^{\Lambda_{m}}=z_{1}^{\lambda_{m 1}} z_{2}^{\lambda_{m 2}} \ldots z_{k}^{\lambda_{m k}}$ where $\lambda_{m, 1}, \ldots, \lambda_{m, k}$ are all positive integers. Let $\beta_{m}=-\log \left|z^{\Lambda_{m}}\right|^{2}$ and $\phi_{k}=\beta_{m}^{2} \tau_{m}^{*} \omega_{\Delta^{*}}$. Then $\beta_{m} \sim \beta$ and

$$
\frac{1}{\beta^{2}} \sum_{i=1}^{k-1} \phi_{i}+\frac{1}{\beta} \omega_{m}+\tau_{m}^{*} \omega_{\Delta^{*}} \sim \frac{1}{\beta^{2}} \sum_{i=1}^{k} \phi_{i}+\frac{1}{\beta} \omega_{m} .
$$

By Lemma (5.3.1), $\sum_{i=1}^{k} \phi_{i} \sim \phi$, provided that the matrix of multiplicities

$$
\Lambda^{\prime}=\left(\begin{array}{ccccc}
1 & 0 & \ldots & 0 & \delta_{1} \\
0 & 1 & \ldots & 0 & \delta_{2} \\
\vdots & \vdots & \ddots & \vdots & \vdots \\
0 & 0 & \ldots & 1 & \delta_{k-1} \\
\lambda_{m, 1} & \lambda_{m, 2} & \ldots & \lambda_{m, k-1} & \lambda_{m, k}
\end{array}\right)
$$


corresponding to the forms $\phi_{1}, \ldots, \phi_{k}$ has nonzero determinant. It is easily shown that

$$
\operatorname{det} \Lambda^{\prime}=\lambda_{m, k}-\sum_{i=1}^{k-1} \lambda_{m, i} \delta_{i} .
$$

We now use our careful calculations of the properties of the matrix $T=T_{m}(D, \tilde{E})$ in $\S 3$. Recall from $\S 8.3$ and $\S 8.2$ that $\lambda_{m, i}=t_{m, j_{i}}$ and $\lambda_{m, k}=t_{m, j_{k}}=t_{m m}$, and note that $\delta_{i}=\delta_{j_{i}, m}$. By Lemma (3.7.3),

$$
t_{m m}=1+\sum_{j=1}^{m-1} t_{m j} \delta_{j m},
$$

where the integers $t_{m j}$ and $\delta_{j m}$ are all nonnegative. Then

$$
\begin{aligned}
\operatorname{det} \Lambda^{\prime} & =t_{m m}-\sum_{i=1}^{k-1} t_{m, j_{i}} \delta_{j_{i}, m} \\
& \geq t_{m m}-\sum_{j=1}^{m-1} t_{m j} \delta_{j m} \\
& =1 .
\end{aligned}
$$

\section{(8.7) Modified Poincaré metrics.}

Proof of (8.4.2). Part (ii) of this theorem follows from Proposition (5.5.1). We now prove part (i). Recall from (8.6.8) that

$$
\begin{aligned}
\pi^{*} \omega_{m-1} \sim \frac{\sqrt{-1}}{\pi}\left(d z_{k} \wedge d \bar{z}_{k}\right. & +d z_{C} \wedge d \bar{z}_{C} \\
& \left.+\left(z_{f} d z_{k}+z_{k} d z_{f}\right) \wedge\left(\bar{z}_{f} d \bar{z}_{k}+\bar{z}_{k} d \bar{z}_{f}\right)\right)
\end{aligned}
$$

where the repeated index $f$ indicates summation over $\Gamma-\{k\}$. Then $\pi^{*} \omega_{m-1}+\frac{\sqrt{-1}}{\pi} d z_{f} \wedge d \bar{z}_{f}$ is positive and hence locally quasi-isometric to $\omega_{m}$. Substituting, we obtain

$$
\begin{aligned}
\omega_{P, m} & =\omega_{S, m}+\omega_{m} \\
\sim \pi^{*} \omega_{S, m-1} & +\frac{\sqrt{-1}}{\pi} \frac{1}{\left.|\log | z_{1} z_{2} \ldots z_{k}\right|^{2} \mid} d z_{f} \wedge d \bar{z}_{f}+\tau_{m}^{*} \omega_{\Delta^{*}} \\
& +\pi^{*} \omega_{m-1}+\frac{\sqrt{-1}}{\pi} d z_{f} \wedge d \bar{z}_{f}
\end{aligned}
$$




$$
\begin{aligned}
& \sim \pi^{*}\left(\omega_{S, m-1}+\omega_{m-1}\right)+\frac{\sqrt{-1}}{\pi} d z_{f} \wedge d \bar{z}_{f}+\tau_{m}^{*} \omega_{\Delta^{*}} \\
& =\pi^{*} \omega_{P, m-1}+\frac{\sqrt{-1}}{\pi} d z_{f} \wedge d \bar{z}_{f}+\tau_{m}^{*} \omega_{\Delta^{*}}
\end{aligned}
$$

\section{(8.8) Metrics in the case $C_{i} \subset D_{i-1}$.}

Proof of (8.4.4). The statement of Theorem (8.4.4) allowed for the possibility that $D_{j}$ might have several connected components, but it is sufficient to prove the theorem in the case that $D_{j}$ is connected, since each blow-up $\pi_{i}$ is a biholomorphism away from its exceptional divisor $E_{i}$. This simplification allows us to avoid having to work with submatrices of multiplicity matrices corresponding to individual connected components of $D_{j}$. For the rest of this proof we will assume that $C_{i} \subset D_{i-1}$ for $2 \leq i \leq m$.

We continue to use the notation of $\S 8$.3. We may write $\omega_{S, m}$ as

$$
\omega_{S, m}=r_{0} \pi_{m, 0}^{*} \omega_{0}+\sum_{i=1}^{m} r_{i} \nu_{m, i}
$$

for some integers $r_{0}, r_{1}, \ldots, r_{m}$. The forms

$$
\nu_{m, i}=\pi_{m, i}^{*} \nu_{i}=-\frac{\sqrt{-1}}{2 \pi} \partial \bar{\partial} \log \left(\log \left\|\hat{s}_{m, i}\right\|^{2}\right)^{2}
$$

are Poincaré-type $(1,1)$-forms corresponding to the divisors $D_{m, i}=$ $\pi_{m, i}^{*} D_{i}$ and have the usual decompositions $\nu_{m, i}=\mu_{i}+\eta_{i}$ as in (5.1.2). In local coordinates, the function $\beta_{i}=-\log \left\|\hat{s}_{m, i}\right\|^{2}$ is given by

$$
\beta_{i}=-\log \left(\left|z^{\Lambda_{i}}\right|^{2} g_{i}\right)
$$

for some bounded positive $C^{\infty}$ function $g_{i}$. If $C_{i} \subset D_{i-1}$ for $2 \leq i \leq$ $m$, the calculation of the quasi-isometry class of $\omega_{S, m}$ is greatly simplified, because in this case all entries of the matrix $\Lambda$ are positive, by Proposition (3.7.4), and consequently $\beta_{i} \sim \beta \sim-\log \left|z_{1} z_{2} \ldots z_{k}\right|^{2}$ for all $i$.

Applying Lemma (8.6.2) inductively in this case, it is easy to show that there are positive integers $r_{i}\left(r_{i}\right.$ a sufficiently large multiple of $\left.r_{i+1}\right)$, such that

$$
r_{0} \pi_{m, 0}^{*} \omega_{0}+\sum_{i=1}^{m} r_{i} \mu_{i} \sim \pi_{m, 0}^{*} \omega_{0}+\frac{1}{\beta} \omega_{m}
$$


By Proposition (5.2.4),

$$
\frac{a}{\beta} \omega_{m}+\eta_{i} \sim \frac{1}{\beta} \omega_{m}+\tau_{i}^{*} \omega_{\Delta^{*}}
$$

locally, for any positive constant $a$. Then locally

$$
\begin{aligned}
r_{0} \pi_{m, 0}^{*} \omega_{0}+\sum_{i=1}^{m} r_{i} \nu_{m, i} & \sim \pi_{m, 0}^{*} \omega_{0}+\frac{1}{\beta} \omega_{m}+\sum_{i=1}^{m} \tau_{i}^{*} \omega_{\Delta^{*}} \\
& \sim \pi_{m, 0}^{*} \omega_{0}+\frac{1}{\beta} \omega_{m}+\frac{1}{\beta^{2}} \sum_{i=1}^{m} \beta_{i}^{2} \tau_{i}^{*} \omega_{\Delta^{*}} \text { since } \beta_{i} \sim \beta .
\end{aligned}
$$

Recall from $\S 8.3$ that the $m \times k$ matrix $\Lambda$ has rank $k$. We may apply Lemma (5.3.1) to the sum of the forms $\phi_{i}=\beta_{i}^{2} \tau_{i}^{*} \omega_{\Delta^{*}}$ to obtain

$$
\sum_{i=1}^{m} \beta_{i}^{2} \tau_{i}^{*} \omega_{\Delta^{*}} \sim \phi
$$

This shows that $\omega_{S, m}$ is homogeneous in the sense of (1.8.4).

Recall that $\omega_{S, 1} \sim \pi_{1}^{*} \omega_{0}+\omega_{\text {Sap }}$ (Corollary (7.2.2)) and if $\operatorname{dim} C_{1}=$ 0 then $\omega_{S, 1} \sim \omega_{\text {Sap }}$ (Corollary (7.2.3)). If $\omega_{S, 1} \sim \omega_{\text {Sap }}$, then we may neglect the term $\pi_{m, 0}^{*} \omega_{0}$ in all successive blow-ups, and we obtain the local quasi-isometry

$$
\omega_{S, m} \sim \frac{1}{\beta^{2}} \phi+\frac{1}{\beta} \omega_{m}
$$

which describes a Saper metric.

To obtain the quasi-isometry $\omega_{P, m} \sim \omega_{P, \text { hom }}$, use Proposition (5.5.1).

§. Homogeneous Saper metrics and proof of Theorem II. Let $M$ be a compact complex Kähler manifold and let $X$ be a reduced compact analytic subspace of $M$. In $\S 9.1$ we will show how to construct a homogeneous Saper metric on the nonsingular set of $X$. This metric has the advantage that it is more easily described in local coordinates than the metrics of Theorem (8.4.1), but the disadvantage that there is not a natural decomposition into terms corresponding to each of the blow-up maps used to resolve $X$.

In $\S 9.2$ we will show that an incomplete metric on $M-X_{\text {sing }}$ which determines an embedded resolution of singularities of $X$ is associated in a very simple way with a certain complete Kähler modified 
Saper metric on $M-X_{\text {sing. }}$ This result, together with Corollary (10.2.4) on fine sheaves, constitutes our second main result, Theorem II of $\S 2.2$.

(9.1) Complete Kähler homogeneous Saper metrics. Let $M=$ $M_{0}$ be a compact complex Kähler manifold with Kähler form $\omega$ and let $X$ be a reduced compact analytic subspace of $M$. Suppose that $\left\{\pi_{j}: M_{j} \rightarrow M_{j-1}\right\}$ is a finite sequence of blow-ups along smooth centres $C_{j} \subset M_{j-1}$ which resolves the singularities of $X$ and such that $C_{j}$ has normal crossings with the total exceptional divisor $D_{j-1}$ of the composite map $\pi_{j, 0}: M_{j} \rightarrow M_{0}$. Let $\tilde{M}=M_{m}$ be the final blow-up, let $\pi=\pi_{m, 0}$ be the composite map from $\tilde{M}$ to $M$, and let $D=D_{m}$ be the total exceptional divisor of $\pi$. The strict transform $\tilde{X}$ of $X$ in $\tilde{M}$ is smooth and has normal crossings with $D$, and $\tilde{X}-(\tilde{X} \cap D)$ is isomorphic to $X-X_{\text {sing. A metric on } \tilde{M}-D \text { induces }}$ a metric on $X-X_{\text {sing }}$.

In $\S 8$ we constructed complete Kähler modified Saper metrics for a sequence of blow-ups of this type. We showed that if for $2 \leq j \leq m$ the centre $C_{j}$ is either contained in the total exceptional divisor $D_{j-1}$ or is disjoint from $D_{j-1}$, our Kähler form $\omega_{S, m}$ on $\tilde{M}-D$ is homogeneous, i.e.

$$
\omega_{S, m} \sim \pi^{*} \omega+\omega_{\text {Sap }}
$$

where $\omega_{\text {Sap }}$ is the fundamental form of a distinguished Saper metric on $\tilde{M}-D$. Now we will show that we may obtain such a metric even when we remove this requirement.

Recall that the irreducible components $\tilde{E}_{m, 1}, \ldots, \tilde{E}_{m, m}$ of $D$ are the strict transforms of the exceptional divisors $E_{j}=\pi_{j}^{-1}\left(C_{j}\right)$.

THEOREM 9.1.1. Let $\mathcal{D}_{1}, \ldots, \mathcal{D}_{m}$ be effective divisors on $\tilde{M}$ with the same support as $D$, i.e. such that

$$
\mathcal{D}_{i}=\sum_{j=1}^{m} b_{i j} \tilde{E}_{m, j}
$$

for some positive integers $b_{i j}$. For each divisor $\mathcal{D}_{i}$, let $H_{i}$ be a hermitian metric on the line bundle $\mathcal{L}_{i}=\left[\mathcal{D}_{i}\right]$ and let $S_{i}$ be a section of $\mathcal{L}_{i}$ such that $\left(S_{i}\right)=\mathcal{D}_{i}$ and $\left\|S_{i}\right\|^{2}<1$ on $\tilde{M}$. Let $\nu_{i}$ be the associated Poincaré-type $(1,1)$-form

$$
\nu_{i}=-\frac{\sqrt{-1}}{2 \pi} \partial \bar{\partial} \log \left(\log \left\|S_{i}\right\|^{2}\right)^{2} .
$$




\section{Suppose that}

i. the matrix of multiplicities $\left(b_{i j}\right)$ is nonsingular, and

ii. the forms

$$
\psi_{j}=l \pi^{*} \omega-c_{1}\left(\left[\mathcal{D}_{j}\right], H_{j}\right)
$$

are positive for all sufficiently large integers $l$.

Then the form

$$
\omega_{S}=l \pi^{*} \omega+\sum_{i=1}^{m} \nu_{i}
$$

is the fundamental form of a complete Kähler homogeneous Saper metric on $\tilde{M}-D$.

Proof. The construction is similar to that of $\S 8$. The form $\omega_{S}$ is $d-$ closed so we need only show that it is positive and has the required local quasi-isometry class.

We decompose $\nu_{j}$ as $\nu_{j}=\mu_{j}+\eta_{j}$ as usual and note that

$$
\mu_{j}=-\frac{2}{\beta_{j}} c_{1}\left(\left[\mathcal{D}_{j}\right], H_{j}\right)
$$

where $\beta_{j}=-\log \left\|S_{j}\right\|^{2}$.

Consider any point $q \in \tilde{M}$. If $q \notin D$ then $\pi^{*} \omega$ is positive and $\nu_{j}$ is bounded in a neighbourhood of $q$ so $l \pi^{*} \omega$ dominates $\nu_{j}$ for large l. Suppose that $q \in D$ and let $\tilde{E}_{1}, \ldots, \tilde{E}_{k}$ be the components of $D$ passing through $q$. Each $\tilde{E}_{i}$ is one of the strict transforms $\tilde{E}_{m, \alpha}$. The divisors $D$ and $\mathcal{D}_{j}$ have the same irreducible components so $\tilde{E}_{1}, \ldots, \tilde{E}_{k}$ are also the components of $\mathcal{D}_{j}$ passing through $q$. Let $\lambda_{j i}$ be the multiplicity of $\tilde{E}_{i}$ in $\mathcal{D}_{j}$. The matrix $\Lambda=\left(\lambda_{j i}\right)$ is the $m \times k$ submatrix of the matrix $\left(b_{i j}\right)$ corresponding to the divisors $\tilde{E}_{1}, \ldots, \tilde{E}_{k}$. The rank of $\Lambda$ is $k$.

Let $\left(z_{1}, \ldots, z_{n}\right)$ be normal coordinates for the divisors $\tilde{E}_{i}$ in a neighbourhood $U$ of $q$ and let $\beta=-\log \left|z_{1} z_{2} \ldots z_{k}\right|^{2}$. Near $q$ we have

$$
\beta_{j} \sim-\log \left|z_{1}^{\lambda_{j 1}} z_{2}^{\lambda_{j 2}} \ldots z_{k}^{\lambda_{j k}}\right|^{2} \sim \beta
$$

By assumption (ii), the forms

$$
\psi_{j}=l \pi^{*} \omega+\frac{1}{2} \beta_{j} \mu_{j}
$$


are positive for all sufficiently large integers $l$. Then

$$
l \pi^{*} \omega+\mu_{j} \sim \pi^{*} \omega+\frac{1}{\beta} \frac{\sqrt{-1}}{\pi} \sum_{i=1}^{n} d z_{i} \wedge d \bar{z}_{i}
$$

for $z_{1}, \ldots, z_{k}$ close enough (but not equal) to 0 . Let $\tau_{j}: U \rightarrow \Delta$ be the monomial map given by

$$
\tau_{j}\left(z_{1}, \ldots, z_{n}\right)=z_{1}^{\lambda_{j 1}} z_{2}^{\lambda_{j 2}} \ldots z_{k}^{\lambda_{j k}} .
$$

It follows from Proposition (5.2.4) that

$$
l \pi^{*} \omega+\mu_{j}+\eta_{j} \sim \pi^{*} \omega+\frac{1}{\beta} \frac{\sqrt{-1}}{\pi} \sum_{i=1}^{n} d z_{i} \wedge d \bar{z}_{i}+\tau_{j}^{*} \omega_{\Delta^{*}}
$$

and from Lemma (5.3.3) that

$$
\sum_{j=1}^{m} \tau_{j}^{*} \omega_{\Delta^{*}} \sim \frac{\sqrt{-1}}{\pi} \frac{1}{\left(\log \left|z_{1} z_{2} \ldots z_{k}\right|^{2}\right)^{2}} \sum_{i=1}^{k} \frac{d z_{i} \wedge d \bar{z}_{i}}{\left|z_{i}\right|^{2}}
$$

because the matrix $\Lambda$ has positive entries and rank $k$. Then

$$
\begin{aligned}
\omega_{S}=l \pi^{*} \omega & +\sum_{j=1}^{m} \nu_{j} \\
\sim \pi^{*} \omega+ & \frac{\sqrt{-1}}{\pi}\left(\frac{1}{\left(\log \left|z_{1} z_{2} \ldots z_{k}\right|^{2}\right)^{2}} \sum_{i=1}^{k} \frac{d z_{i} \wedge d \bar{z}_{i}}{\left|z_{i}\right|^{2}}\right. \\
& \left.+\frac{1}{\left.|\log | z_{1} z_{2} \ldots z_{k}\right|^{2} \mid} \sum_{i=1}^{n} d z_{i} \wedge d \bar{z}_{i}\right)
\end{aligned}
$$

for $z_{1}, \ldots, z_{k}$ close enough (but not equal) to 0 . This shows that $\omega_{S}$ is positive for $l \gg 0$ and that $\omega_{S} \sim \pi^{*} \omega+\omega_{\text {Sap }}$, i.e. $\omega_{S}$ is homogeneous. Completeness follows from Proposition (6.1.1).

CoRollary 9.1.2. There exists a complete Kähler homogeneous Saper metric on $\tilde{M}-D$ and hence on $X-X_{\text {sing }} \cong \tilde{X}-(\tilde{X} \cap D)$.

Proof. Let $\mathcal{D}_{1}, \ldots, \mathcal{D}_{m}$ and $H_{1}, \ldots, H_{m}$ be the divisors and metrics of Proposition (4.4.1). These divisors and metrics satisfy conditions (i) and (ii) of the previous theorem. For each $i$ we may choose a section $S_{i}$ of the line bundle $\mathcal{L}_{i}=\left[\mathcal{D}_{i}\right]$ such that $\left(S_{i}\right)=\mathcal{D}_{i}$ and such 
that $\left\|S_{i}\right\|^{2}<1$ on $\tilde{M}$. Let $\nu_{1}, \ldots, \nu_{m}$ be the associated Poincarétype $(1,1)$-forms. Then the form

$$
\omega_{S}=l \pi^{*} \omega+\sum_{i=1}^{m} \nu_{i}
$$

is the fundamental form of a complete Kähler homogeneous Saper metric on $\tilde{M}-D$.

(9.2) Incomplete and complete Kähler metrics. We will now describe a simple relationship between certain incomplete and complete metrics on $X-X_{\text {sing }}$.

First note that in Theorem (9.1.1) we needed only a single Poincarétype $(1,1)$-form $\nu_{i}$ to obtain completeness because each divisor $\mathcal{D}=$ $\mathcal{D}_{i}$ has the same support as $D$. Furthermore, we assumed that the Chern form $c_{1}([\mathcal{D}], H)$ corresponding to the metric $H=H_{i}$ had the property that

$$
\psi=l \pi^{*} \omega-c_{1}([\mathcal{D}], H)
$$

was positive on $\tilde{M}$ for all sufficiently large integers $l$. We may write this assumption in terms of a section $S$ of $[\mathcal{D}]$ such that $(S)=\mathcal{D}$ and $\|S\|^{2}<1$ as

$$
\psi=l \pi^{*} \omega-\frac{\sqrt{-1}}{2 \pi} \partial \bar{\partial}\left(-\log \|S\|^{2}\right)>0
$$

on $\tilde{M}$. The form $\psi$ is a Kähler form on $\tilde{M}$ which determines an incomplete metric on $\tilde{M}-D \cong M-X_{\text {sing }}$. The completion of $M-X_{\text {sing }}$ under this metric is $\tilde{M}$. Recall (definition (2.2.1)) that we say that this incomplete metric on $M-X_{\text {sing }}$ determines an embedded resolution of the singularities of $X$. We will now show that each such metric corresponds to a complete Kähler modified Saper metric on $M-X_{\text {sing }} \cong \tilde{M}-D$.

THEOREM 9.2.1. Let $\mathcal{D}$ be an effective divisor on $\tilde{M}$ with the same support as $D$, let $S$ be a section of the line bundle $\mathcal{L}=[\mathcal{D}]$ such that $(S)=\mathcal{D}$ and $\|S\|^{2}<1$, and let $H$ be a metric on $\mathcal{L}$ such that for all sufficiently large integers $l$

$$
\tilde{\Omega}=l \pi^{*} \omega-\frac{\sqrt{-1}}{2 \pi} \partial \bar{\partial}\left(-\log \|S\|^{2}\right)
$$


determines a Kähler metric on $\tilde{M}$. Then the $(1,1)$-form

$$
\Omega_{S}=l \pi^{*} \omega-\frac{\sqrt{-1}}{2 \pi} \partial \bar{\partial} \log \left(-\log \|S\|^{2}\right)^{2}
$$

determines a complete Kähler modified Saper metric on $\tilde{M}-D$ such that

$$
\pi^{*} \omega \stackrel{\sim}{\leq} \Omega_{S} \stackrel{\sim}{\leq} \pi^{*} \omega+\omega_{\text {Sap }}
$$

and

$$
\frac{1}{-\log \|S\|^{2}} \tilde{\Omega} \tilde{\leq} \Omega_{S}
$$

Proof. Since $\Omega_{S}$ is positive away from $D$ for $l \gg 0$, we need only prove the given quasi-isometries on neighbourhoods of points of $D$. We write $\Omega_{S}$ as $l \pi^{*} \omega+\nu=l \pi^{*} \omega+\mu+\eta$ where

$$
\begin{aligned}
& \nu=-\frac{\sqrt{-1}}{2 \pi} \partial \bar{\partial} \log \left(-\log \|S\|^{2}\right)^{2} \\
& \mu=-\frac{1}{\beta} \frac{\sqrt{-1}}{\pi} \partial \bar{\partial}\left(-\log \|S\|^{2}\right)=-\frac{2}{\beta} c_{1}([\mathcal{D}], H), \\
& \eta=\frac{\sqrt{-1}}{\pi} \frac{\partial \beta \wedge \bar{\partial} \beta}{\beta^{2}}, \quad \text { and } \\
& \beta=-\log \|S\|^{2} .
\end{aligned}
$$

Then

$$
\tilde{\Omega}=l \pi^{*} \omega+\frac{1}{2} \beta \mu
$$

and

$$
l \pi^{*} \omega+\mu \sim l \pi^{*} \omega+\frac{1}{\beta} \tilde{\Omega}>0 .
$$

Next we calculate the local quasi-isometry class of

$$
\Omega_{S}=l \pi^{*} \omega+\nu=l \pi^{*} \omega+\mu+\eta .
$$

Choose normal coordinates $\left(z_{1}, \ldots, z_{n}\right)$ near $q \in \operatorname{supp} D=\operatorname{supp} \mathcal{D}$. Locally the divisor $\mathcal{D}$ is given by the equation $z_{1}^{\lambda_{1}} z_{2}^{\lambda_{2}} \ldots z_{k}^{\lambda_{k}}=0$ for some positive integers $\lambda_{1}, \ldots, \lambda_{k}$. Let $\tau$ be the local monomial map given by

$$
\tau\left(z_{1}, \ldots, z_{n}\right)=z_{1}^{\lambda_{1}} z_{2}^{\lambda_{2}} \ldots z_{k}^{\lambda_{k}} .
$$


Using Proposition (5.2.4) we get locally

$$
l \pi^{*} \omega+\mu+\eta \sim \pi^{*} \omega+\frac{1}{\beta} \tilde{\Omega}+\tau^{*} \omega_{\Delta^{*}}
$$

which is always positive. This shows that $\Omega_{S}$ determines a Kähler modified Saper metric on $\tilde{M}-D$ and that $\Omega_{S} \geq(1 / \beta) \tilde{\Omega}$. Moreover $\Omega_{S}$ is bounded above by the Kähler form $\omega_{S}$ of a homogeneous Saper metric, i.e. a Kähler form $\omega_{S}$ such that $\omega_{S} \sim \pi^{*} \omega+\omega_{\text {Sap. }}$. To construct such an $\omega_{S}$, we proceed as in the proof of Corollary (9.1.2) but note that for some $i$ we may replace $\mathcal{D}_{i}$ by $\mathcal{D}$ and preserve the properties needed to apply Theorem (9.1.1).

We use Proposition (6.1.1) to get completeness.

Corollary 9.2.2. There exist a divisor $\mathcal{D}$ on $\tilde{M}$ with the same support as $D$, a section $S$ of the line bundle $\mathcal{L}=[\mathcal{D}]$, and a metric $H$ on $\mathcal{L}$ such that for all sufficiently large integers $l$

i. the $(1,1)$-form

$$
\tilde{\Omega}=l \pi^{*} \omega-\frac{\sqrt{-1}}{2 \pi} \partial \bar{\partial}\left(-\log \|S\|^{2}\right)
$$

determines a Kähler metric on $\tilde{M}$ and

ii. the $(1,1)$-form

$$
\Omega_{S}=l \pi^{*} \omega-\frac{\sqrt{-1}}{2 \pi} \partial \bar{\partial} \log \left(-\log \|S\|^{2}\right)^{2}
$$

determines a complete Kähler modified Saper metric on $\tilde{M}-D$ such that

$$
\pi^{*} \omega \stackrel{\sim}{\leq} \Omega_{S} \stackrel{\sim}{\leq} \pi^{*} \omega+\omega_{\text {Sap }}
$$

and

$$
\frac{1}{-\log \|S\|^{2}} \tilde{\Omega} \stackrel{\sim}{\leq} \Omega_{S}
$$

Proof. We have many choices for $\mathcal{D}$, since all the Kähler forms we constructed on $\tilde{M}$ by the methods of $\S 4$ may be written in the form (i). For example, let $\mathcal{D}$ be any of the divisors $\mathcal{D}_{j}$ of Corollary (9.1.2) and Proposition (4.4.1). Then

$$
\tilde{\Omega}=\psi_{j}
$$

is positive on $\tilde{M}$ by Proposition (4.4.1) so it determines a Kähler metric on $\tilde{M}$. Now apply Theorem (9.2.1). 
$\S 10$. Fine sheaves. Let $M$ be a compact complex manifold and let $X$ be a reduced compact analytic subspace of $M$. Let $\pi: \tilde{M} \rightarrow$ $M$ be the composite of a finite sequence of blow-ups of the type described in $\S 1$ which resolve the singularities of $X$. Let $D$ be the exceptional divisor of $\pi$. The strict transform $\tilde{X}$ of $X$ in $\tilde{M}$ is smooth and has normal crossings with $D$ and the restriction of $\pi$ to $\tilde{X}-(\tilde{X} \cap D)$ is a biholomorphism onto $X-X_{\text {sing. }}$.

Let $h$ be a hermitian metric on $X-X_{\text {sing }} \cong \tilde{X}-(\tilde{X} \cap D)$. Let $\mathcal{S}_{0}$ be the complex of presheaves on $X$ whose sections over any open set $U$ in $X$ are smooth measurable differential forms $\phi$ on $U \cap\left(X-X_{\text {sing }}\right)$ such that both $\phi$ and $d \phi$ are $L_{2}$-bounded with respect to $h$. Let $\mathcal{S}$ be the associated complex of sheaves on $X$. Similarly, let $\tilde{\mathcal{S}}_{0}$ be the complex of presheaves on $\tilde{X}$ whose sections over any open set $U$ in $\tilde{X}$ are smooth measurable differential forms $\phi$ on $U-(U \cap D)$ such that both $\phi$ and $d \phi$ are $L_{2}$-bounded with respect to $h$, and let $\tilde{\mathcal{S}}$ be the associated complex of sheaves on $\tilde{X}$.

We will show that if $h$ is bounded below by the restriction to $X-X_{\text {sing }}$ of a hermitian metric on $M$, then $\mathcal{S}$ is a complex of fine sheaves. In particular, if $h$ is a modified Saper or modified Poincaré metric then the complex of sheaves $\mathcal{S}$ on $X$ is fine. Similarly, if $h$ is bounded below by the restriction to $\tilde{X}-(\tilde{X} \cap D)$ of a hermitian metric on $\tilde{X}$, then $\tilde{\mathcal{S}}$ is also fine. In particular, if $h$ is a modified Poincaré metric then the complex of sheaves $\tilde{\mathcal{S}}$ on $\tilde{X}$ is fine.

We first review some technical material concerning $L_{2}$-norms of forms.

(10.1) Comparison of $L_{2}$-bounded forms in two metrics. Let $h_{A}$ and $h_{B}$ be two hermitian metrics on an $m$-dimensional complex manifold $Y$. Let $W$ be a coordinate neighbourhood of a point $y$ in $Y$. After shrinking $W$ if necessary, we may choose $C^{\infty}$ sections $t_{1}, \ldots, t_{m}$ of the tangent bundle $T W$ which are linearly independent at each point of $W$ and orthonormal with respect to $h_{A}$. We call $t_{1}, \ldots, t_{m}$ an orthonormal frame for $h_{A}$. Let $\tau_{1}, \ldots, \tau_{m}$ be dual sections of the cotangent space $T^{*} W$. We call $\tau_{1}, \ldots, \tau_{m}$ an orthonormal coframe for $h_{A}$. Let $G=\left(G_{i j}\right)$ be the $C^{\infty}$ matrix for $h_{B}$ with respect to the frame $t_{1}, \ldots, t_{m}$, i.e. $\left\langle t_{i}, t_{j}\right\rangle_{B}=G_{i j}$, where $G_{i j}$ is some $C^{\infty}$ function on $W$. Then $\left\langle\tau_{i}, \tau_{j}\right\rangle_{B}=\left(G^{-1}\right)_{j i}$. The matrix $G$ is hermitian positive definite at every point, so there is a (constant) unitary matrix $U$ 
such that

$$
U G(y) U^{*}=D
$$

where $U^{*}=\bar{U}^{t}$ and $D$ is a (constant) diagonal matrix with positive diagonal entries $d_{i}$.

REMARK 10.1.1. At each point $y$ in $Y$, we have $h_{B} \geq h_{A}$ if and only if $d_{i} \geq 1$ for all $i$.

Next we construct an orthonormal frame and coframe for $h_{A}$ with respect to which the matrix for $h_{B}$ at the point $y$ is diagonal. Let

$$
e_{i}=\sum_{j=1}^{m} U_{i j} t_{j}
$$

and

$$
\xi_{i}=\sum_{j=1}^{m} \tau_{j}\left(U^{-1}\right)_{j i} .
$$

Then $e_{1}, \ldots, e_{m}$ is another orthonormal frame for $h_{A}$ and $\xi_{1}, \ldots, \xi_{m}$ is another orthonormal coframe for $h_{A}$. The matrix for $h_{B}$ with respect to the frame $\left\{e_{i}\right\}$ is

$$
\hat{D}=U G U^{*}
$$

where $\left\langle e_{i}, e_{j}\right\rangle_{B}=\hat{D}_{i j}$ and $\left\langle\xi_{i}, \xi_{j}\right\rangle_{B}=\left(\hat{D}^{-1}\right)_{j i}$. Note that $\hat{D}(y)=D$. At the point $y$ we have

$$
\begin{aligned}
& \left\langle e_{i}(y), e_{j}(y)\right\rangle_{A}=\delta_{i j}, \quad\left\langle e_{i}(y), e_{j}(y)\right\rangle_{B}=d_{i} \delta_{i j}, \\
& \left\langle\xi_{i}(y), \xi_{j}(y)\right\rangle_{A}=\delta_{i j}, \quad \text { and }\left\langle\xi_{i}(y), \xi_{j}(y)\right\rangle_{B}=\frac{1}{d_{i}} \delta_{i j} \text {, }
\end{aligned}
$$

where $\delta_{i j}=1$ if $i=j$ and $\delta_{i j}=0$ if $i \neq j$. We may extend the collection of tangent vectors $\left\{\frac{1}{\sqrt{d_{i}}} e_{i}(y)\right\}$ to an orthonormal frame $\left\{e_{i}^{\prime}\right\}$ for $h_{B}$ on $W$ and the collection of cotangent vectors $\left\{\sqrt{d_{i}} \xi_{i}(y)\right\}$ to an orthonormal coframe $\left\{\xi_{i}^{\prime}\right\}$ for $h_{B}$. Let $C$ be the matrix for the change of frame

$$
e_{i}^{\prime}=\sum_{j} C_{i j} e_{j} \quad \text { and } \quad \xi_{i}^{\prime}=\sum_{j} \xi_{j}\left(C^{-1}\right)_{j i} .
$$

Then

$$
C \hat{D} C^{*}=I \quad \text { and } \quad C(y)=\sqrt{D^{-1}}
$$


i.e. $C(y)$ is the diagonal matrix whose diagonal elements are $\frac{1}{\sqrt{d_{2}}}$. Volume forms for $h_{A}$ and $h_{B}$ are

$$
d V_{A}=\frac{\sqrt{-1}}{2 m !} \xi_{1} \wedge \bar{\xi}_{1} \wedge \ldots \wedge \xi_{m} \wedge \bar{\xi}_{m}
$$

and

$$
\begin{aligned}
d V_{B} & =\frac{\sqrt{-1}}{2 m !} \xi_{1}^{\prime} \wedge \bar{\xi}_{1}^{\prime} \wedge \ldots \wedge \xi_{m}^{\prime} \wedge \bar{\xi}_{m}^{\prime} \\
& =\operatorname{det} C^{-1} \operatorname{det} \bar{C}^{-1} d V_{A} \\
& =\operatorname{det} \hat{D} d V_{A} .
\end{aligned}
$$

Recall that $\hat{D}(y)=D$, so that $\operatorname{det} \hat{D}(y)=d_{1} d_{2} \ldots d_{m}$.

We now compare $L_{2}$-norms with respect to $h_{A}$ and $h_{B}$. Let $\psi$ be a $p$-form which is locally of the form

$$
\psi=\sum_{i_{1}<\ldots<i_{p}} \alpha_{\left(i_{\imath}, \ldots, i_{p}\right)} \xi_{i_{1}} \wedge \ldots \wedge \xi_{i_{p}}=\sum_{I} \alpha_{I} \xi_{I}=\sum_{I} \beta_{I} \xi_{I}^{\prime}
$$

Similar calculations can be done for $(p, q)$-forms. We have

$$
\langle\psi, \psi\rangle_{A}=\sum_{I}\left|\alpha_{I}\right|^{2} \quad \text { and } \quad\langle\psi, \psi\rangle_{B}=\sum_{I}\left|\beta_{I}\right|^{2} .
$$

Integrating over $Y$ gives the $L_{2}$-norms

$$
(\psi, \psi)_{A}=\int_{Y}\langle\psi, \psi\rangle_{A} d V_{A} \quad \text { and } \quad(\psi, \psi)_{B}=\int_{Y}\langle\psi, \psi\rangle_{B} d V_{B}
$$

where $d V_{B}=\operatorname{det} \hat{D} d V_{A}$ locally.

To compare $L_{2}$-norms with respect to $h_{A}$ and $h_{B}$ we will compare the local expressions for $\langle\psi, \psi\rangle_{A}$ and $\langle\psi, \psi\rangle_{B} \operatorname{det} \hat{D}$.

For example, if $p=0$ and $\psi=\alpha$, then

$$
\langle\psi, \psi\rangle_{A}=\langle\psi, \psi\rangle_{B}=|\alpha|^{2}
$$

and $\psi$ is locally $B-L_{2}$-bounded if and only if $\sqrt{\operatorname{det} \hat{D}} \psi$ is locally $A$ - $L_{2}$-bounded.

If $p=m$ and $\psi=\alpha \xi_{1} \wedge \ldots \wedge \xi_{m}$, then

$$
\langle\psi, \psi\rangle_{B} \operatorname{det} \hat{D}=\frac{|\alpha|^{2}}{\operatorname{det} \hat{D}} \operatorname{det} \hat{D}=\langle\psi, \psi\rangle_{A}
$$


and $\psi$ is $B-L_{2}$-bounded if and only if $\psi$ is $A-L_{2}$-bounded.

If $p=1$ and $\psi=\sum \alpha_{i} \xi_{i}=\sum \beta_{i} \xi_{i}^{\prime}$ then at the point $y$,

$$
\beta_{i}=\frac{1}{\sqrt{d_{i}}} \alpha_{i}
$$

If $h_{B} \geq h_{A}$ then $d_{i} \geq 1$ for all $i$ so

$$
\sum_{i}\left|\beta_{i}\right|^{2}=\sum_{i} \frac{1}{d_{i}}\left|\alpha_{i}\right|^{2} \leq \sum_{i}\left|\alpha_{i}\right|^{2}
$$

i.e.

$$
\langle\psi, \psi\rangle_{B} \leq\langle\psi, \psi\rangle_{A}
$$

Similarly,

$$
\begin{aligned}
\langle\psi, \psi\rangle_{B} \operatorname{det} \hat{D}(y) & =\sum_{i} \frac{\prod_{j} d_{j}}{d_{i}}\left|\alpha_{i}\right|^{2} \\
& \geq\langle\psi, \psi\rangle_{A}
\end{aligned}
$$

at each point $y$ so

$$
(\psi, \psi)_{B} \geq(\psi, \psi)_{A}
$$

if $h_{B} \geq h_{A}$.

(10.2) Fine sheaves. Let $Y$ be a reduced compact analytic subspace of an $n$-dimensional compact Kähler manifold $M$ and let $Z$ be a complex analytic subspace of $Y$ containing the singular set of $Y$. Let $h_{M}$ be a hermitian metric on $Y-Z$ induced from a hermitian metric on $M$. Let $h$ be another hermitian metric on $Y-Z$ and let $\mathcal{S}_{0}$ be the complex of presheaves on $Y$ whose sections over any open set $U$ in $Y$ are smooth measurable differential forms $\phi$ on $U \cap(Y-Z)$ such that both $\phi$ and $d \phi$ are $L_{2}$-bounded with respect to $h$. Let $\mathcal{S}$ be the associated complex of sheaves on $Y$. We will show that if $h \geq h_{M}$ on $Y-Z$ then $\mathcal{S}$ is a complex of fine sheaves.

The main technical result we need is the following proposition. Recall that by a $C^{\infty}$ function on $Y$ we mean the restriction to $Y$ of a $C^{\infty}$ function on $M$.

Proposition 10.2.1. Let $U$ be an open set in $Y$ and let $\phi$ be a smooth measurable differential form on $U \cap(Y-Z)$ such that 
$\phi$ is $L_{2}$-bounded with respect to $h$. Let $f$ be a $C^{\infty}$ function on $Y$. Suppose that $h \geq h_{M}$ on $Y-Z$. Then df $\wedge \phi$ is $L_{2}$-bounded with respect to $h$.

We will work in terms of frames and coframes of the type described in $\S 10.1$ above, letting $h_{A}=h_{M}$ and $h_{B}=h$. More specifically, for each $y \in Y-Z$ we may choose a coordinate neighbourhood $W$ on which we have an orthonormal frame $\left\{e_{i}\right\}$ and coframe $\left\{\xi_{i}\right\}$ for $h_{A}$, and an orthonormal frame $\left\{e_{i}^{\prime}\right\}$ and coframe $\left\{\xi_{i}^{\prime}\right\}$ for $h_{B}$ such that $\left\langle e_{i}, e_{j}\right\rangle_{B}=\hat{D}_{i j}$ and $\hat{D}(y)=D$ is a diagonal matrix with positive diagonal entries $d_{i}$. The statement that $h_{B} \geq h_{A}$ means that every eigenvalue $d_{i}$ of $D$ satisfies $d_{i} \geq 1$. Then for every 1-form $\psi$, we have $\langle\psi, \psi\rangle_{B} \leq\langle\psi, \psi\rangle_{A}$. In particular, $\langle d f, d f\rangle_{B} \leq\langle d f, d f\rangle_{A}$. But $\langle d f, d f\rangle_{A}$ is bounded because $f$ is a $C^{\infty}$ function on $Y$ and $h_{A}$ comes from a metric on $M$. Choose $K>0$ such that $\langle d f, d f\rangle_{A} \leq K$ everywhere on $Y$.

Suppose that $\phi$ is a $p$-form.

LEMMA 10.2.2. $\langle d f \wedge \phi, d f \wedge \phi\rangle_{B} \leq K\left(\begin{array}{c}n \\ p+1\end{array}\right)\langle\phi, \phi\rangle_{B}$.

Proof. In terms of the coframe $\left\{\xi_{i}^{\prime}\right\}$, let

$$
d f=\sum_{j} f_{j} \xi_{j}^{\prime} \quad \text { and } \quad \phi=\sum_{I} \phi_{I} \xi_{I}^{\prime}
$$

Then

$$
d f \wedge \phi=\sum_{|J|=p+1}\left(\sum_{\{j, I\}=J} f_{j} \phi_{I}(-1)^{\operatorname{sgn}(j, I)}\right) \xi_{J}^{\prime}
$$

and

$$
\begin{aligned}
\langle d f \wedge \phi, d f \wedge \phi\rangle_{B} & =\sum_{|J|=p+1}\left|\sum_{\{j, I\}=J} f_{j} \phi_{I}(-1)^{\operatorname{sgn}(j, I)}\right|^{2} \\
& \leq \sum_{|J|=p+1}\left(\sum_{j \in J}\left|f_{j}\right|^{2}\right)\left(\sum_{I \subset J}\left|\phi_{I}\right|^{2}\right) \\
& \leq \sum_{|J|=p+1}\langle d f, d f\rangle_{B}\langle\phi, \phi\rangle_{B} \\
& =\left(\begin{array}{c}
n \\
p+1
\end{array}\right)\langle d f, d f\rangle_{B}\langle\phi, \phi\rangle_{B}
\end{aligned}
$$




$$
\begin{aligned}
& \leq\left(\begin{array}{c}
n \\
p+1
\end{array}\right)\langle d f, d f\rangle_{A}\langle\phi, \phi\rangle_{B} \\
& \leq K\left(\begin{array}{c}
n \\
p+1
\end{array}\right)\langle\phi, \phi\rangle_{B} .
\end{aligned}
$$

We now conclude the proof of Proposition (10.2.1). The $h-L_{2^{-}}$ norm of $d f \wedge \phi$ on $U^{\prime}=U \cap(Y-Z)$ is

$$
\begin{aligned}
(d f \wedge \phi, d f \wedge \phi)_{B} & =\int_{U^{\prime}}\langle d f \wedge \phi, d f \wedge \phi\rangle_{B} d V_{B} \\
& \leq K\left(\begin{array}{c}
n \\
p+1
\end{array}\right) \int_{U^{\prime}}\langle\phi, \phi\rangle_{B} d V_{B} \\
& <\infty
\end{aligned}
$$

since the form $\phi$ on $U^{\prime}$ is $L_{2}$-bounded with respect to $h=h_{B}$.

We use Proposition (10.2.1) to prove our main result about fine sheaves.

Proposition 10.2.3. If $h \geq h_{M}$ on $Y-Z$, then $\mathcal{S}$ is a complex of fine sheaves.

Proof. It is sufficient to prove that multiplication by a $C^{\infty}$ function determines a morphism of presheaves $\mathcal{S}_{0} \rightarrow \mathcal{S}_{0}$. Let $\phi$ be a section of $\mathcal{S}_{0}$ over an open set $U$ in $Y$, i.e. $\phi$ is a smooth measurable differential form on $U \cap(Y-Z)$ such that $\phi$ and $d \phi$ are $L_{2}$-bounded with respect to $h$. We will show that for any $C^{\infty}$ function $f$ on $Y$, the forms $f \phi$ and $d(f \phi)$ are also $h$ - $L_{2}$-bounded. Now $d(f \phi)=d f \wedge \phi+f d \phi$, and $f \phi$ and $f d \phi$ are $h-L_{2}$-bounded because $f$ is bounded. By Proposition (10.2.1), $d f \wedge \phi$ is also $h-L_{2}$-bounded.

Thus multiplication by a function which is $C^{\infty}$ on $Y$ determines a presheaf morphism from $\mathcal{S}_{0}$ to $\mathcal{S}_{0}$. The induced map from $\mathcal{S}$ to $\mathcal{S}$ is a sheaf morphism, so $\mathcal{S}$ admits partitions of unity and is a complex of fine sheaves.

Now we return to the situation of the introduction to $\S 10$, namely that $X$ is a reduced compact analytic subspace of $M, \pi: \tilde{M} \rightarrow M$ is a composite of blow-ups which resolves the singularities of $X, D$ is 
the exceptional divisor of $\pi$, and $\tilde{X}$ is the (smooth) strict transform of $X$ in $\tilde{M}$.

Corollary 10.2.4. Let $h$ be a modified Saper metric on $\tilde{M}-D$. Then the associated complexes of $L_{2}$ sheaves on $X$ and $M$ are fine.

Proof. Recall that every modified Saper metric on $\tilde{M}-D \cong$ $M-X_{\text {sing }}$ is bounded below by a metric induced from $M$ and then use Proposition (10.2.3).

Corollary 10.2.5. Let $h_{P}$ be a modified Poincaré metric on $\tilde{M}-D$. Then the associated complexes of $L_{2}$ sheaves on $X, M, \tilde{X}$, and $\tilde{M}$ are all fine.

Proof. Recall that every modified Poincaré metric on $\tilde{M}-D \cong$ $M-X_{\text {sing }}$ is bounded below by a metric on $M-X_{\text {sing }}$ induced from $M$ and also by a metric on $\tilde{M}-D$ induced from $\tilde{M}$.

Appendix: Tubular neighbourhood construction. The following tubular neighbourhood constructions are based on Clemens [CL, §5] and are used in the proof of Proposition (4.1.1).

Let $X$ be a complex manifold and let $Y$ be a compact complex submanifold. Let $N=N_{Y / X}$ be the normal bundle of $Y$ in $X$. Let $F$ be a vector bundle over $X$ and, for any subset $Z$ of $X$, let $F_{Z}$ be the restriction of $F$ to $Z$.

Proposition A.1. There exist an open neighbourhood $U$ of $Y$ in $X$ and $a C^{\infty}$ projection $\tau: U \rightarrow Y$ such that

$\left(^{*}\right) \tau$ is surjective and the fibres of $\tau$ are holomorphic submanifolds of $U$, transverse to $Y$.

Proposition A.2. There exist an open neighbourhood $U$ of $Y$ in $X$ and $C^{\infty}$ maps $\tau: U \rightarrow Y$ and $\psi: U \rightarrow N$ such that $\tau$ satisfies ${ }^{*}$ ) of Proposition 1 and such that

i. the restriction of $\psi$ to $Y$ is the natural identification of $Y$ with the zero section of $N$,

ii. $\psi$ is a diffeomorphism onto its image, and

iii. the restriction of $\psi$ to any fibre of $\tau: U \rightarrow Y$ is a biholomorphism onto an open set in the corresponding fibre of $N$ over $Y$. 
Proposition A.3. There exist an open neighbourhood $U$ of $Y$ and $C^{\infty}$ maps $\tau: U \rightarrow Y$ and $\rho: F_{U} \rightarrow \tau^{*} F_{Y}$ such that $\tau$ satisfies $\left(^{*}\right)$ of Proposition 1 and such that

i. the restriction of $\rho$ to $F_{Y}$ is the identity map,

ii. $\rho$ is a $C^{\infty}$ vector bundle isomorphism, and iii. the following diagram commutes.

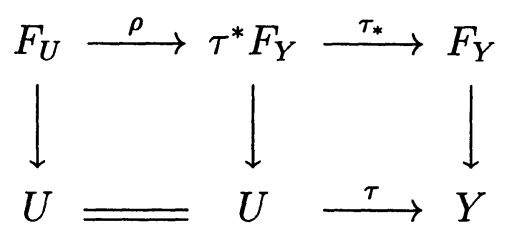

All these propositions are proved in a similar manner, by piecing together local maps using a partition of unity on $Y$ and the appropriate transition functions.

Proof of Proposition A.1. Let $\left\{U_{\alpha}\right\}$ be a finite open cover of $Y$ in $X$ with the following properties:

i. The closure $\overline{U_{\alpha}}$ of $U_{\alpha}$ in $X$ is compact.

ii. On $U_{\alpha}$ there are holomorphic coordinates $z_{\alpha, 1}, \ldots, z_{\alpha, n}$ such that the set $Y_{\alpha}=Y \cap U_{\alpha}$ is given by $z_{\alpha, 1}=\ldots=z_{\alpha, k}=0$ and such that $z_{\alpha, k+1}, \ldots, z_{\alpha, n}$ are holomorphic coordinates on $Y_{\alpha}$.

Define a map $v_{\alpha}: U_{\alpha} \times Y_{\alpha} \rightarrow \mathbf{C}^{n-k}$ by

$$
v_{\alpha}(x, y)=\left(z_{\alpha, i}(x)-z_{\alpha, i}(y)\right)_{i=k+1, \ldots, n}
$$

where $x \in U_{\alpha}, y \in Y_{\alpha}$, and $z_{\alpha, i}(p)$ represents the value of the holomorphic coordinate $z_{\alpha, i}$ at the point $p$.

For each $y$ in $Y_{\alpha}$, the restriction of $v_{\alpha}$ to $Y_{\alpha} \times\{y\} \subset U_{\alpha} \times Y_{\alpha}$ is holomorphic in $x$ and gives a system of holomorphic coordinates on $Y_{\alpha}$, centered at $y$. For $y$ in $Y_{\alpha} \cap Y_{\beta}$, let $J_{\alpha, \beta}(y)$ be the Jacobian matrix relating $v_{\alpha}$-coordinates to $v_{\beta}$-coordinates at $y$.

Let $\left\{\sigma_{\alpha}\right\}$ be a $C^{\infty}$ partition of unity on $Y$, subordinate to the cover $\left\{Y_{\alpha}\right\}$ of $Y$. Pick any Riemannian metric on $X$ and let

$$
d=\min _{\alpha} \operatorname{dist}\left(\operatorname{supp}\left(\sigma_{\alpha}\right), U_{\alpha}^{c}\right) .
$$

Since $\operatorname{supp}\left(\sigma_{\alpha}\right)$ is closed and contained in $U_{\alpha}$, and $\left\{U_{\alpha}\right\}$ is a finite cover of $Y$ with $\overline{U_{\alpha}}$ compact in $X$, we have $0<d<\infty$. Let $W_{\alpha}$ be 
the neighbourhood of $Y_{\alpha} \times Y_{\alpha}$ in $U_{\alpha} \times Y_{\alpha}$ defined by

$$
W_{\alpha}=\left\{(x, y) \in U_{\alpha} \times Y_{\alpha}: \operatorname{dist}(x, y)<\frac{d}{2}\right\} .
$$

In order for products of the form $\sigma_{\gamma} v_{\gamma} J_{\gamma, \alpha}$ to be defined on all of $W_{\alpha}$, we make the convention that $v_{\gamma}$ is 0 outside $U_{\gamma} \times Y_{\gamma}$ and $J_{\gamma, \alpha}$ is 0 outside $Y_{\alpha} \cap Y_{\gamma}$.

Define maps

$$
A_{\alpha}: W_{\alpha} \rightarrow \mathbf{C}^{n-k}
$$

by

$$
A_{\alpha}(x, y)=\sum_{\gamma} \sigma_{\gamma}(y) v_{\gamma}(x, y) J_{\gamma, \alpha}(y)
$$

Notice that if $(x, y) \in W_{\alpha}$ and $\sigma_{\gamma}(y) \neq 0$, then

$$
\operatorname{dist}\left(x, \operatorname{supp}\left(\sigma_{\gamma}\right)\right)<\frac{d}{2}
$$

so $x \in U_{\gamma}$. Then $A_{\alpha}$ is a $C^{\infty}$ function and the restriction of $A_{\alpha}$ to $\left(U_{\alpha} \times\{y\}\right) \cap W_{\alpha}$ is holomorphic.

On $W_{\alpha} \cap W_{\beta}$ we have

$$
\begin{aligned}
A_{\beta}(x, y) J_{\beta, \alpha}(y) & =\sum_{\gamma} \sigma_{\gamma}(y) v_{\gamma}(x, y) J_{\gamma, \beta}(y) J_{\beta, \alpha}(y) \\
& =\sum_{\gamma} \sigma_{\gamma}(y) v_{\gamma}(x, y) J_{\gamma, \alpha}(y) \\
& =A_{\alpha}(x, y)
\end{aligned}
$$

so $A_{\alpha}(x, y)=0$ if and only if $A_{\beta}(x, y)=0$.

For each $y_{0} \in Y_{\alpha}$, the restriction of $A_{\alpha}$ to $\left\{y_{0}\right\} \times Y_{\alpha}$ gives a holomorphic coordinate system on a neighbourhood of $y_{0}$ in $Y_{\alpha}$. By the implicit function theorem, there exist $C^{\infty}$ functions $u_{k+1}, \ldots, u_{n}$ on a neighbourhood of $y_{0}$ in $X$, such that in a neighbourhood of $\left(y_{0}, y_{0}\right)$ in $W_{\alpha}, A_{\alpha}(x, y)=0$ if and only if $z_{\alpha, i}(y)=u_{i}(x)$ for $k+1 \leq$ $i \leq n$. Locally, define a map $\tau$ by

$$
\tau(x)=\left(u_{i}(x)\right)_{k+1 \leq i \leq n}
$$

so that $A_{\alpha}(x, y)=0$ if and only if $y=\tau(x)$. Since on $W_{\alpha} \cap W_{\beta}$, $A_{\alpha}(x, y)=0$ if and only if $A_{\beta}(x, y)=0$, the map $\tau$ may be extended to a $C^{\infty}$ function on a neighbourhood $W$ of $Y$ in $X$, with 
the property: for each $\gamma, A_{\gamma}(x, y)=0$ if and only if $y=\tau(x)$. Since the restriction of $A_{\alpha}$ to $\left(U_{\alpha} \times\{y\}\right) \cap W_{\alpha}$ is holomorphic and the restriction to $Y_{\alpha} \times\left\{y_{0}\right\}$ is nondegenerate in a neighbourhood of $\left(y_{0}, y_{0}\right)$, there is a neighbourhood $U\left(y_{0}\right)$ of $y_{0}$ in $W$ such that for $y$ in $Y \cap U\left(y_{0}\right)$, the fibre $\tau^{-1}(y) \cap U\left(y_{0}\right)$ of $\tau$ is a holomorphic manifold of dimension $k$, transverse to $Y$. Cover $Y$ by a finite number of such neighbourhoods, let $U$ be their union, and restrict $\tau$ to be the projection $\tau: U \rightarrow Y$.

Proof of Proposition A.2. Let $\tau: U \rightarrow Y$ be as in Proposition 1. Let $\left\{U_{\alpha}\right\}$ be a finite open covering of $Y$ in $X$ with properties (i) and (ii) of the proof of Proposition 1 and such that:

iii. There are local trivializations of the normal bundle

$$
\begin{gathered}
\phi_{\alpha}:\left.N\right|_{Y_{\alpha}} \rightarrow Y_{\alpha} \times \mathbf{C}^{k} \\
\phi_{\alpha}(\xi)=\left(y, \xi_{\alpha}\right)
\end{gathered}
$$

with transition functions $g_{\alpha, \beta}$ on $Y_{\alpha} \cap Y_{\beta}$, such that $g_{\alpha, \beta}(y) \xi_{\beta}=\xi_{\alpha}$. Note that $g_{\alpha, \beta}$ is a $k \times k$ matrix so $\xi_{\alpha}$ and $\xi_{\beta}$ should be regarded as column vectors. In terms of holomorphic coordinate systems $z_{\alpha}$ and $z_{\beta}$ on $U_{\alpha}$ and $U_{\beta}$, the matrix $g_{\alpha, \beta}$ is given by

$$
g_{\alpha, \beta}=\left(\frac{\partial z_{\alpha, i}}{\partial z_{\beta, j}}\right)_{1 \leq i, j \leq k}
$$

Let $Y_{\alpha}=Y \cap U_{\alpha}$. Shrinking the sets $U_{\alpha}$ and $U$ if necessary, we may assume that the union of the sets $U_{\alpha}$ is $U$ and that $\tau^{-1}\left(Y_{\alpha}\right)=U_{\alpha}$.

To give a map $\psi: U \rightarrow N$, it is enough to give maps

$$
\psi_{\alpha}: U_{\alpha} \rightarrow Y_{\alpha} \times \mathbf{C}^{k}
$$

of the form

$$
\psi_{\alpha}(x)=\left(\tau(x), \xi_{\alpha}(x)\right)
$$

such that $g_{\alpha, \beta}(\tau(x)) \xi_{\beta}(x)=\xi_{\alpha}(x)$ for $x \in U_{\alpha} \cap U_{\beta}$.

Let $\left\{\sigma_{\alpha}\right\}$ be a partition of unity on $Y$, subordinate to the cover $\left\{Y_{\alpha}\right\}$. Let $\tilde{z}_{\gamma}=\left(z_{\gamma, i}\right)_{i=1, \ldots, k}$. We use the convention that $g_{\alpha, \gamma}(y)=0$ if $y \notin Y_{\alpha} \cap Y_{\gamma}$ and $z_{\gamma}(x)=0$ if $x \notin U_{\gamma}$. Define maps

$$
\xi_{\alpha}: U_{\alpha} \rightarrow \mathbf{C}^{k}
$$


by

$$
\xi_{\alpha}(x)=\sum_{\gamma} \sigma_{\gamma}(\tau(x)) g_{\alpha, \gamma}(\tau(x)) \tilde{z}_{\gamma}(x)
$$

Notice that $\xi_{\alpha}$ is $C^{\infty}$ since $\operatorname{supp}\left(\sigma_{\gamma}\right) \subset Y_{\gamma}$. On $U_{\alpha} \cap U_{\beta}$ we have

$$
\begin{aligned}
g_{\alpha, \beta}(\tau(x)) \xi_{\beta}(x) & =\sum_{\gamma} \sigma_{\gamma}(\tau(x)) g_{\alpha, \beta}(\tau(x)) g_{\beta, \gamma}(\tau(x)) \tilde{z}_{\gamma}(x) \\
& =\sum_{\gamma} \sigma_{\gamma}(\tau(x)) g_{\alpha, \gamma}(\tau(x)) \tilde{z}_{\gamma}(x) \\
& =\xi_{\alpha}(x)
\end{aligned}
$$

so that the maps $\psi_{\alpha}(x)=\left(\tau(x), \xi_{\alpha}(x)\right)$ do indeed define a $C^{\infty}$ map $\psi: U \rightarrow N$.

Next we check that $\psi$ has the desired properties. If $y \in Y$ then $\tau(y)=y$ and $\tilde{z}_{\gamma}(y)=0$ for all $\gamma$, so the restriction of $\psi$ to $Y$ is the natural map of $Y$ onto the zero section of $N$. The restriction of $\psi$ to the fibre $U_{y}=\tau^{-1}(y)$ of $\tau$ is given by

$$
\psi(x)=\left(y, \xi_{\alpha}(x)\right)
$$

where

$$
\xi_{\alpha}(x)=\sum_{\gamma} \sigma_{\gamma}(y) g_{\alpha, \gamma}(y) \tilde{z}_{\gamma}(x),
$$

so $\left.\psi\right|_{U_{y}}$ is a holomorphic map to the fibre $N_{y}$ of $N$. To see that (after shrinking $U$ if necessary) $\psi$ is a diffeomorphism onto its image and the restriction of $\psi$ to any fibre of $\tau$ is a local biholomorphism, note that in a neighbourhood of $y$ on the fibre $U_{y}$ of $\tau, \xi_{\alpha}$ is a nondegenerate map to $\mathbf{C}^{k}$.

Proof of Proposition A.3. Let $\tau: U \rightarrow Y$ be as in Proposition 1 and let $r=\operatorname{rank}(F)$. Let $\left\{U_{\alpha}\right\}$ be a finite open covering of $Y$ in $X$ with properties (i) and (ii) of the proof of Proposition 1 and such that:

iii. There are local trivializations of $F$

$$
\begin{gathered}
\phi_{\alpha}:\left.F\right|_{U_{\alpha}} \rightarrow U_{\alpha} \times \mathbf{C}^{r} \\
\phi_{\alpha}(\xi)=\left(x, \xi_{\alpha}\right)
\end{gathered}
$$

with transition functions $g_{\alpha, \beta}$ on $U_{\alpha} \cap U_{\beta}$ such that $g_{\alpha, \beta}(x) \xi_{\beta}=\xi_{\alpha}$. Let $Y_{\alpha}=Y \cap U_{\alpha}$. Shrinking the sets $U_{\alpha}$ and $U$ if necessary, we may 
assume that the union of the sets $U_{\alpha}$ is $U$ and that $\tau^{-1}\left(Y_{\alpha}\right)=U_{\alpha}$. Transition functions for $\tau^{*} F$ on $U_{\alpha} \cap U_{\beta}$ are $g_{\alpha, \beta} \circ \tau$. To give a map $\rho: F_{U} \rightarrow \tau^{*} F_{Y}$, it is enough to give maps

$$
\rho_{\alpha}: U_{\alpha} \times \mathbf{C}^{r} \rightarrow U_{\alpha} \times \mathbf{C}^{r}
$$

of the form

$$
\rho_{\alpha}\left(x, \xi_{\alpha}\right)=\left(x, f_{\alpha}\left(x, \xi_{\alpha}\right)\right)
$$

such that $g_{\alpha, \beta}(\tau(x)) f_{\beta}\left(x, \xi_{\beta}\right)=f_{\alpha}\left(x, \xi_{\alpha}\right)$ for $x \in U_{\alpha} \cap U_{\beta}$.

Let $\left\{\sigma_{\alpha}\right\}$ be a partition of unity on $Y$, subordinate to the covering $\left\{Y_{\alpha}\right\}$. We use the convention that $g_{\alpha, \gamma}(x)=0$ if $x \notin U_{\alpha} \cap U_{\gamma}$. Define maps

$$
f_{\alpha}: U_{\alpha} \times \mathbf{C}^{r} \rightarrow \mathbf{C}^{r}
$$

by

$$
f_{\alpha}\left(x, \xi_{\alpha}\right)=\sum_{\gamma} \sigma_{\gamma}(\tau(x)) g_{\alpha, \gamma}(\tau(x)) g_{\gamma, \alpha}(x) \xi_{\alpha} .
$$

Since $g_{\gamma, \alpha}(x) \xi_{\alpha}=\xi_{\gamma}$ we have

$$
f_{\alpha}\left(x, \xi_{\alpha}\right)=\sum_{\gamma} \sigma_{\gamma}(\tau(x)) g_{\alpha, \gamma}(\tau(x)) \xi_{\gamma} .
$$

On the restriction of $F$ to $U_{\alpha} \cap U_{\beta}$ we have

$$
\begin{aligned}
g_{\alpha, \beta}(\tau(x)) f_{\beta}\left(x, \xi_{\beta}\right) & =\sum_{\gamma} \sigma_{\gamma}(\tau(x)) g_{\alpha, \beta}(\tau(x)) g_{\beta, \gamma}(\tau(x)) \xi_{\gamma} \\
& =\sum_{\gamma} \sigma_{\gamma}(\tau(x)) g_{\alpha, \gamma}(\tau(x)) \xi_{\gamma} \\
& =f_{\alpha}\left(x, \xi_{\alpha}\right) .
\end{aligned}
$$

Notice that $f_{\alpha}$ is $C^{\infty}$ since $\operatorname{supp}\left(\sigma_{\gamma}\right) \subset Y_{\gamma}$.

Finally, we check that the map $\rho$ defined by the functions $f_{\alpha}$ has the required properties. If $y \in Y_{\alpha}$ then $\tau(y)=y$ and

$$
\begin{aligned}
f_{\alpha}\left(y, \xi_{\alpha}\right) & =\sum_{\gamma} \sigma_{\gamma}(y) g_{\alpha, \gamma}(y) \xi_{\gamma} \\
& =\sum_{\gamma} \sigma_{\gamma}(y) g_{\alpha, \gamma}(y) g_{\gamma, \alpha}(y) \xi_{\alpha} \\
& =\left(\sum_{\gamma} \sigma_{\gamma}(y)\right) \xi_{\alpha} \\
& =\xi_{\alpha}
\end{aligned}
$$


so $\rho$ is the identity map on $F_{Y}$. After shrinking $U$ if necessary, the map $\rho: F_{U} \rightarrow \tau^{*} F_{Y}$ will be a $C^{\infty}$ vector bundle isomorphism. By construction, $\rho$ commutes with projection to $U$.

\section{REFERENCES}

[BM1] E. Bierstone and P. Milman, Uniformization of analytic spaces, J. Amer. Math. Soc., 2 (1989), 801-836.

[BM2] - A simple constructive proof of Canonical Resolution of Singularities, Effective Methods in Algebraic Geometry, Birkhäuser, Progr. Math., vol. 94, Boston, (1991), 11-30.

[CL] C. H. Clemens, Degeneration of Kähler Manifolds, Duke Math. J., 44 (1977), 214-290.

[GH] P. Griffiths and J. Harris, Principles of Algebraic Geometry, WileyInterscience, New York, 1978.

[GM] M. Goresky and R. MacPherson, Intersection Homology II, Invent. Math., 71 (1983), 77-129.

[GrM] C. Grant and P. Milman, Metrics for singular analytic spaces, preprint (Dec. 1990), 1-33.

[H] H. Hironaka, Resolution of singularities of an algebraic variety over a field of characteristic zero: I, II, Ann. Math., 79 (1964), 109-326.

[O] T. Ohsawa, On the $L^{2}$ cohomology of complex spaces, Math. Z., 209 (1992), 519-530.

[S1] L. Saper, $L_{2}$-cohomology and intersection homology of certain algebraic varieties with isolated singularities, Invent. Math., 82 (1985) 207-255.

[S2] — , L $L_{2}$-cohomology of Kähler varieties with isolated singularities, J. Diff. Geom., 36 (1992), 89-161.

[W] R. O. Wells, Differential Analysis on Complex Manifolds, Springer-Verlag, New York, 1980.

[Z1] S. Zucker, Hodge theory with degenerating coefficients: $L_{2}$ cohomology in the Poincaré metric, Ann. of Math., 109 (1979), 415-476.

[Z2] $\longrightarrow, L_{2}$ Cohomology of Warped Products and Arithmetic Groups, Invent. Math., 70 (1982), 169-218.

Received September 24, 1992. The first author was partially supported by a grant from the Naval Academy Research Council. The second author wes partially supported by a grant from NSERC. 
ANNAPOLIS, MD 21402-5002

E-mail address: cgg@sma.usna.navy.MIL

AND

UNIVERSITY OF TORONTO

TORONTO, ONTARIO M53 1A1

E-mail address: milman@math.toronto.edu 


\section{PACIFIC JOURNAL OF MATHEMATICS}

Founded by E. F. Beckenbach (1906-1982) and F. Wolf (1904-1989)

\section{EDITORS}

Sun-Yung Alice Chang (Managing Editor)

University of California

Los Angeles, CA 90095-1555

pacific@math.ucla.edu

F. Michael Christ

University of California

Los Angeles, CA 90095-1555

christ@math.ucla.edu

Thomas Enright

University of California

San Diego, La Jolla, CA 92093

tenright@ucsd.edu

Nicholas Ercolani

University of Arizona

Tucson, AZ 85721

ercolani@math.arizona.edu
Robert Finn

Stanford University

Stanford, CA 94305

finn@gauss.stanford.edu

Vaughan F. R. Jones

University of California

Berkeley, CA 94720

vfr@math.berkeley.edu

Steven Kerckhoff

Stanford University

Stanford, CA 94305

spk@gauss.stanford.edu

\author{
Martin Scharlemann \\ University of California \\ Santa Barbara, CA 93106 \\ mgscharl@math.ucsb.edu \\ Gang Tian \\ Courant Institute \\ New York University \\ New York, NY 10012-1100 \\ tiang@taotao.cims.nyu.edu
V. S. Varadarajan
University of California
Los Angeles, CA 90095-1555
vsv@math.ucla.edu

\section{SUPPORTING INSTITUTIONS}

\section{CALIFORNIA INSTITUTE OF TECHNOLOGY NEW MEXICO STATE UNIVERSITY OREGON STATE UNIVERSITY \\ STANFORD UNIVERSITY \\ UNIVERSITY OF ARIZONA \\ UNIVERSITY OF BRITISH COLUMBIA \\ UNIVERSITY OF CALIFORNIA UNIVERSITY OF HAWAII}

\author{
UNIVERSITY OF MONTANA \\ UNIVERSITY OF NEVADA, RENO \\ UNIVERSITY OF OREGON \\ UNIVERSITY OF SOUTHERN CALIFORNIA \\ UNIVERSITY OF UTAH \\ UNIVERSITY OF WASHINGTON \\ WASHINGTON STATE UNIVERSITY
}

The supporting Institutions listed above contribute to the cost of publication of this Journal, but they are not owners or publishers and have no responsibility for its contents or policies.

Manuscripts must be prepared in accordance with the instructions provided on the inside back cover.

The Pacific Journal of Mathematics (ISSN 0030-8730) is published monthly except for July and August. Regular subscription rate: $\$ 215.00$ a year (10 issues). Special rate: $\$ 108.00$ a year to individual members of supporting institutions.

Subscriptions, orders for back issues published within the last three years, and changes of subscribers address should be sent to Pacific Journal of Mathematics, P.O. Box 4163, Berkeley, CA 94704-0163, U.S.A. Prior back issues are obtainable from Kraus Periodicals Co., Route 100, Millwood, NY 10546.

The Pacific Journal of Mathematics at the University of California, c/o Department of Mathematics, 981 Evans Hall, Berkeley, CA 94720 (ISSN 0030-8730) is published monthly except for July and August. Second-class postage paid at Berkeley, CA 94704, and additional mailing offices. POSTMASTER: send address changes to Pacific Journal of Mathematics, P.O. Box 6143, Berkeley, CA 94704-0163.

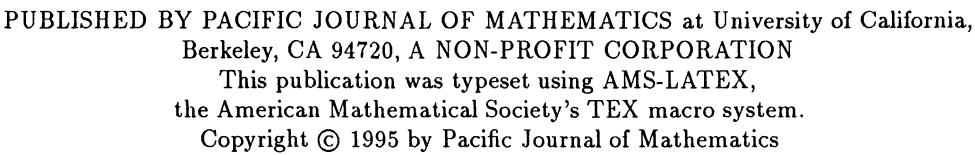




\section{PACIFIC JOURNAL OF MATHEMATICS}

Volume $168 \quad$ No. $1 \quad$ March 1995

Compact contractible $n$-manifolds have arc spines $(n \geq 5)$

FREDRIC DAVIS ANCEL and CRAIG R. GUILBAULT

On uniform homeomorphisms of the unit spheres of certain Banach lattices

\section{FOUAD CHAATIT}

Applications of subordination chains to starlike mappings in $\mathbb{C}^{n}$

MARTIN CHUAQUi

The $L^{p}$ theory of standard homomorphisms

FEREIDOUn GHAHRAMANI and SANDY GRABINER

Metrics for singular analytic spaces

Caroline Galt Grant and Pierre Milman

Maps on infra-nilmanifolds

KYUNG BAI LEE

Hardy spaces and oscillatory singular integrals. II

YIBIAO PAN

Continuity of convex hull boundaries

Linda Jo Goldway KeEn and Caroline SERIES 